\title{
Álgebras de caminhos generalizadas com relações e suas representações
}

\author{
Viktor Chust Bugno Pires de Almeida
}

\author{
DisSERTAÇÃO APRESENTADA \\ $A O$ \\ Instituto De Matemática e Estatística \\ DA \\ Universidade DE SÃo PaUlo \\ PARA \\ OBTENÇÃO DO TÍTULO \\ $\mathrm{DE}$ \\ Mestre em CiênCIAS \\ Programa: Matemática \\ Orientador: Prof. Dr. Flávio Ulhoa Coelho
}

Durante o desenvolvimento deste trabalho o autor recebeu auxílio financeiro da FAPESP

São Paulo, novembro de 2020 


\section{Álgebras de caminhos generalizadas com relações e suas representações}

Esta é a versão original da dissertação elaborada pelo candidato Viktor Chust Bugno Pires de Almeida, tal como submetida à Comissão Julgadora. 


\section{Agradecimentos}

Este projeto de Mestrado recebeu apoio financeiro através do processo n ${ }^{0}$ 2018/18123-5, Fundação de Amparo à Pesquisa do Estado de São Paulo (FAPESP). As opiniões, hipóteses e conclusões ou recomendações expressas neste material são de responsabilidade do autor e não necessariamente refletem a visão da FAPESP. Gostaria de gentilmente agradecer pelo apoio da FAPESP nos últimos anos.

Gostaria também de fazer um agradecimento especial a meu orientador, o Prof. Dr. Flávio Ulhoa Coelho, a quem sempre deverei respeito e gratidão. 


\section{Resumo}

CHUST, V. Álgebras de caminhos generalizadas com relações e suas representações. 2020. 88 f. Dissertação (Mestrado) - Instituto de Matemática e Estatística, Universidade de São Paulo, São Paulo, 2020.

O conceito de álgebras de caminhos generalizadas (abreviadas aqui por ACG), tal como tratado neste texto, foi introduzido por F. U. Coelho e S. X. Liu em [7]. O objetivo da presente dissertação é aprofundar o conhecimento sobre tais álgebras e suas representações, elencando tanto resultados já existentes na literatura quanto novas abordagens a serem apresentadas aqui. Seja $\Gamma$ uma aljava (também chamada de quiver, em inglês), e seja $\mathcal{A}=\left\{A_{i}: i \in \Gamma_{0}\right\}$ uma família de álgebras, onde $\Gamma_{0}$ é o conjunto dos vértices de $\Gamma$. Uma álgebra de caminhos generalizada $k(\Gamma, \mathcal{A})$ é definida como sendo o espaço vetorial tendo como base o conjunto de caminhos sobre $\Gamma$ intercalados por elementos das álgebras $A_{i}$ que correspondem a cada vértice. A multiplicação em $k(\Gamma, \mathcal{A})$ é então definida por concatenação de caminhos e usando as multiplicações internas das álgebras $A_{i}$. Outro trabalho que será fundamental aqui é o artigo [6]. Nele, os autores R. M. Ibáñez-Cobos, G. Navarro e J. LópezPeña obtém generalizações para dois teoremas bem conhecidos de P. Gabriel, que originalmente tratam das álgebras de caminhos usuais (ver [3, 4], por exemplo, para uma introdução a esses teoremas). Um dos problemas com o quais lidamos é decidir quando uma álgebra dada é isomorfa a uma ACG de forma não trivial. O tratamento deste problema ganha contornos mais interessantes quando permitimos que as ACGs tenham relações. Adaptando as definições e os resultados de [6] a esse novo contexto, é possível abordar o problema citado acima usando critérios de natureza combinatória. Dada uma ACG $k(\Gamma, \mathcal{A})$, dizemos que uma propriedade de álgebras ou de representações vale localmente se ela vale para cada álgebra que pertence à família $\mathcal{A}$, e dizemos que ela vale globalmente se vale para a álgebra $k(\Gamma, \mathcal{A})$. A relação entre propriedades locais e globais é outro problema relevante que discutiremos aqui. Na literatura, exemplos dessas propriedades aparecem em $[9,15]$. Ainda neste contexto, aprofundando uma discussão presente em [12], é possível descrever as representações de uma ACG que correspondem a módulos simples, projetivos e injetivos.

Palavras-chave: álgebras de caminhos, álgebras de caminhos generalizadas, representações de álgebras, representações de aljavas. 


\section{Abstract}

CHUST, V. Generalized bound path algebras and their representations. 2020. $88 \mathrm{f}$. Dissertation (Master's Degree) - Instituto de Matemática e Estatística, Universidade de São Paulo, São Paulo, 2020.

The concept of generalized path algebras (here abbreviated by GPA), in the way it is treated here, was introduced by F. U. Coelho and S. X. Liu in [7]. The aim of this dissertation is to give a deeper knowledge about these algebras and their representations, listing not only results already existent in the literature but also new approaches which are to be introduced here. Let $\Gamma$ be a quiver, and let $\mathcal{A}=\left\{A_{i}: i \in \Gamma_{0}\right\}$ be a family of algebras, where $\Gamma_{0}$ is the set of vertices of $\Gamma$. A generalized path algebra $k(\Gamma, \mathcal{A})$ is defined as the vector space having as its basis the set of paths over $\Gamma$ interposed by elements from the $A_{i}$ which correspond to each vertex. Multiplication in $k(\Gamma, \mathcal{A})$ is subsequently defined by juxtaposition of paths and using the internal multiplications of the algebras $A_{i}$. Another work which will be fundamental here is the article [6]. There, the authors R. M. Ibáñez-Cobos, G. Navarro and J. López-Peña obtain generalizations of two wellknown theorems due to P. Gabriel, which originally deal with ordinary path algebras (see [3, 4], e.g., for an introduction to these theorems). One of the problems we deal with is to decide whether a given algebra is isomorphic to a GPA in a non-trivial manner. The approach to this problem is even more interesting when we allow the GPA's to have relations. By adapting the definitions and results from [6] to this new context, it is possible to study this problem using criteria which have combinatorial type. Given a GPA $k(\Gamma, \mathcal{A})$, we say that a property of algebras or representations holds locally if it holds for every algebra belonging to the family $\mathcal{A}$, and we say it holds globally if it holds for the algebra $k(\Gamma, \mathcal{A})$. The relationship between local and global properties is another relevant problem to be discussed here. In the literature, examples of these properties appear in $[9,15]$. Still in this context, by a deeper insight into a discussion present in [12], it is possible to describe the representations that correspond to simple, projective and injective modules.

Keywords: path algebras, generalized path algebras, representations of algebras, representations of quivers. 


\section{Conteúdo}

Lista de Abreviaturas $\quad$ xi

Lista de Símbolos $\quad$ xiii

1 Introdução $\quad 1$

2 Conceitos preliminares $\quad 3$

2.1 Suposições iniciais . . . . . . . . . . . . . . . . . . . . . 3

2.2 Noções sobre módulos . . . . . . . . . . . . . . . . . . . . . 8

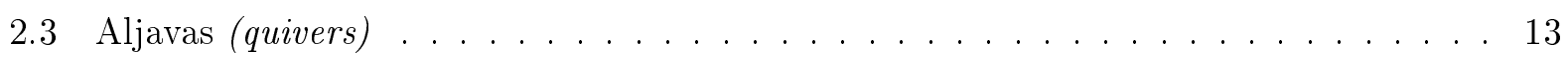

2.4 A categoria das aljavas . . . . . . . . . . . . . . . . . . . 14

2.5 Álgebras de Caminhos . . . . . . . . . . . . . . . . . . . . . . . . . . . . . .

2.6 Os Teoremas de Gabriel . . . . . . . . . . . . . . . . . . . . 18

3 Álgebras de Caminhos Generalizadas (ACG) 23

3.1 Definições . . . . . . . . . . . . . . . . . . . . . 23

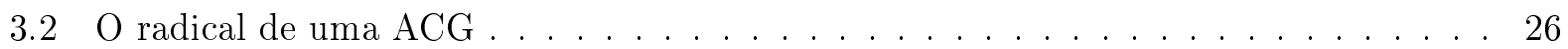

4 Generalização: ACG's com relações $\quad 31$

4.1 Álgebras de Caminhos Generalizadas com Relações . . . . . . . . . . . . . . . . . . . 31

4.2 Realizando uma ACGR como uma álgebra de caminhos . . . . . . . . . . . . . . 32

4.3 Simplificações . . . . . . . . . . . . . . . . . . . . . . 39

4.4 Um critério para simplificabilidade . . . . . . . . . . . . . . . . . . . 40

4.4 .1 Relações de equivalência sobre vértices . . . . . . . . . . . . . . . . . . 40

4.4 .2 Simplificações a partir de relações de equivalência . . . . . . . . . . . . . . . . . 42

4.4 .3 Relações de equivalência a partir de simplificações . . . . . . . . . . . . . . . 43

4.5 Exemplos e aplicações . . . . . . . . . . . . . . . . . . . . . . 45

5 Representações de Álgebras de Caminhos Generalizadas $\quad 49$

5.1 Equivalência entre módulos e representações . . . . . . . . . . . . . . . . . . . . 49

5.2 Módulos simples . . . . . . . . . . . . . . . . . . . . . . . . . 54

5.3 Realizando um $A_{i}$-módulo como um $\Lambda$-módulo $\ldots \ldots \ldots \ldots \ldots \ldots$

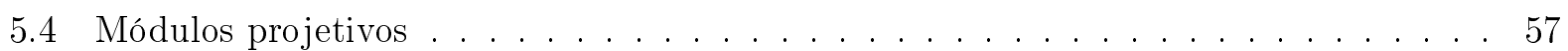

5.5 Álgebra Oposta e Dualidade . . . . . . . . . . . . . . . . . . . . 59

5.6 Módulos injetivos . . . . . . . . . . . . . . . . . . . . . 63 
A Conceitos equivalentes aos de ACG

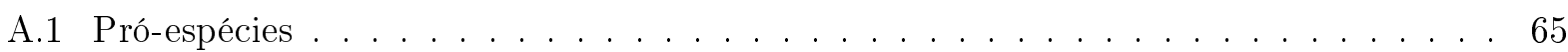

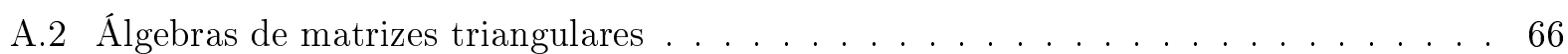

A.3 Álgebras de pseudocaminhos (ou pseudotensoriais) . . . . . . . . . . . . 68

$\begin{array}{ll}\text { Índice } & 70\end{array}$

$\begin{array}{ll}\text { Bibliografia } & 73\end{array}$ 


\section{Lista de Abreviaturas}

AC Álgebra de Caminhos (Path Algebra)

ACR Álgebra de Caminhos com Relações (Bound Path Algebra)

ACG Álgebra de Caminhos Generalizada (Generalized Path Algebra)

ACGR Álgebra de Caminhos Generalizada com Relações (Generalized Bound Path Algebra) 


\section{Lista de Símbolos}

Seguem abaixo notações comumente usadas ao longo deste trabalho:

$k \quad$ um corpo algebricamente fechado

$A \quad$ uma $k$-álgebra de dimensão finita e básica

$Q$ ou $\Gamma \quad$ aljavas

$\mathcal{A} \quad$ uma família de $k$-álgebras

$I$ ou $R \quad$ um conjunto de relações

$k(\Gamma, \mathcal{A}, I) \quad$ a álgebra de caminhos generalizada sobre $\Gamma$ e $\mathcal{A}$ com relações $I$

$\bmod A \quad$ categoria de $A$-módulos à direita finitamente gerados

$A$ - mod categoria de $A$-módulos à esquerda finitamente gerados

rk $K_{0}(A)$ posto do grupo de Grothendieck de $A$ 


\section{Capítulo 1}

\section{Introdução}

O conceito de álgebras de caminhos generalizadas (abreviadas aqui por ACG), tal como tratado neste texto, foi introduzido por F. U. Coelho e S. X. Liu em [7]. O objetivo da presente dissertação é aprofundar o conhecimento sobre tais álgebras e suas representações, elencando tanto resultados já existentes na literatura quanto novas abordagens a serem apresentadas aqui.

Seja $\Gamma$ uma aljava (também chamada de quiver, em inglês), e seja $\mathcal{A}=\left\{A_{i}: i \in \Gamma_{0}\right\}$, onde o conjunto indexador é o conjunto $\Gamma_{0}$ dos vértices de $\Gamma$. Definimos um $\mathcal{A}$-caminho como sendo uma sequência formal $a_{0} \beta_{1} a_{1} \ldots \beta_{n} a_{n}$, onde $\beta_{1} \ldots \beta_{n}$ é um caminho usual sobre $\Gamma, a_{0}$ pertence a $A_{s\left(\beta_{1}\right)}$, onde $s\left(\beta_{1}\right)$ denota o vértice em que a flecha $\beta_{1}$ começa, e para todo $i>0, a_{i}$ pertence a $A_{e\left(\beta_{i}\right)}$, onde $e\left(\beta_{i}\right)$ denota o vértice em que termina a flecha $\beta_{i}$. Analogamente ao que é feito para as álgebras de caminhos usuais, uma álgebra de caminhos generalizada $k(\Gamma, \mathcal{A})$ é definida como sendo o espaço vetorial tendo como base o conjunto de todos $\mathcal{A}$-caminhos, módulo relações que garantem linearidade. Daí a multiplicação em $k(\Gamma, \mathcal{A})$ é definida de forma natural, por concatenação de caminhos e usando as multiplicações internas das álgebras $A_{i}$ que compõem a família $\mathcal{A}$.

No artigo original [7], os autores se concentram em dois problemas básicos: o primeiro é estabelecer certas propriedades de anéis que as ACGs satisfazem. Os resultados lá obtidos consistem na descrição do radical dessas álgebras, e condições necessárias e suficientes para que elas sejam noetherianas ou primas. O segundo problema lida com unicidade, isto é, sobre quais conclusões podemos tirar quando duas ACG's são isomorfas entre si.

Outro trabalho que será fundamental aqui será o artigo [6]. Nele, os autores R. M. IbáñezCobos, G. Navarro e J. López-Peña obtém generalizações para dois teoremas bem conhecidos de P. Gabriel, que originalmente tratavam das álgebras de caminhos usuais (ver [3, 4], por exemplo, para uma introdução a esses teoremas). Com isso, eles são capazes de resolver o seguinte problema:

Problema 1: Dada uma ACG $k(\Gamma, \mathcal{A})$, obter, tal como no Teorema de Gabriel, uma aljava $Q$ e um conjunto de relações $\Omega$ sobre $Q$ tais que $k(\Gamma, \mathcal{A}) \cong k Q / \Omega$. (Ou seja, realizar $k(\Gamma, \mathcal{A})$ como uma álgebra de caminhos usual).

Uma discussões do presente trabalho é o problema inverso, isto é:

Problema 2: Dada uma álgebra $A$, decidir se existe, de forma não trivial, uma aljava $\Gamma$ e uma família de álgebras $\mathcal{A}=\left\{A_{i}: i \in \Gamma_{0}\right\}$ tal que $A \cong k(\Gamma, \mathcal{A})$.

O tratamento do problema 2 ganha contornos mais interessantes quando permitimos que as ACGs tenham relações. Tal expansão do conceito original, para as denominadas álgebras de cami- 
nhos generalizadas com relações (ACGR), será portanto aqui introduzida. (Mas veja também [10]). Uma vez generalizados os resultados de [6] para este novo contexto, somos capazes de descrever dois critérios de natureza combinatória que permitem abordar o problema 2.

Seja agora $P$ uma propriedade relativa a uma álgebra ou às suas representações. Seguindo a terminologia de [9], dada uma ACG $k(\Gamma, \mathcal{A})$, dizemos que $P$ vale globalmente quando ela vale para a álgebra $k(\Gamma, \mathcal{A})$, e dizemos que $P$ vale localmente se ela vale para cada uma das álgebras que pertencem à família $\mathcal{A}$. Com esta terminologia, um importante problema é o seguinte:

Problema 3: Encontrar exemplos de propriedades em que haja relação entre o fato de valerem localmente com o de valerem globalmente.

Já existem na literatura sobre ACG's exemplos de propriedades que satisfazem a condição do problema 3. (Citamos [15, 9], por exemplo). De certa forma, o artigo [6] também faz isso ao generalizar para as ACGs o teorema de Gabriel sobre representações. Neste trabalho, vamos expandir o resultado de [6] para as ACGRs e com isto aprofundar uma discussão que aparece em [12], descrevendo quais são as representações que correspondem a módulos simples, projetivos e injetivos sobre as ACGRs.

Este trabalho está organizado como segue: no capítulo 2, relembramos alguns conceitos sobre álgebras, módulos e aljavas, bem como os teoremas de Gabriel acima referidos. No capítulo 3, lembramos a definição de álgebras de caminhos generalizadas e algumas de suas propriedades básicas. No capítulo 4, estendemos o conceito de ACG, permitindo a introdução de relações na aljava. Generalizamos um dos teoremas principais de [6] para esse contexto, e concluímos o capítulo com os prometidos critérios para o problema 2 acima. No capítulo 5, passamos a estudar as representações das ACGRs. Faremos uma generalização do outro teorema principal de [6], e usaremos isso para descrever as representações simples, projetivas e injetivas sobre uma ACGR. Haverá também um apêndice, onde discutimos conexões entre ACGs e outros conceitos existentes na literatura. 


\section{Capítulo 2}

\section{Conceitos preliminares}

Nesta seção inicial, vamos recordar os conceitos que serão utilizados mais à frente, bem como fixar notações. Os resultados são muitas vezes enunciados sem demonstração aqui. Se o leitor quiser mais detalhes sobre o conteúdo tratado aqui, recomendamos as referências [2, 1], que tratam de Teoria de Módulos, e [3, 4], que introduzem os conceitos de aljavas e álgebras de caminhos.

\subsection{Suposições iniciais}

No presente texto, os principais objetos que serão considerados são as álgebras e os módulos (ou representações) sobre elas. Considerando que o conceito de álgebra pode variar dependendo do autor, entendemos por bem enunciar o que exatamente definimos como álgebra:

Definição 2.1.1. Seja $A$ um conjunto não vazio e seja $k$ um corpo. Dizemos que $A$ é uma álgebra sobre $k$ ou uma $k$-álgebra se $A$ tiver, simultaneamente, uma estrutura de anel (associativo, com unidade) e uma de $k$-espaço vetorial, sendo as duas compatíveis entre si. Mais explicitamente, isso significa dizer que $A$ está equipada com três operações: uma chamada de soma e denotada por $+: A \times A \rightarrow A$, outra chamada de multplicação (interna), com forma $\circ: A \times A \rightarrow A$, e uma última, chamada de multiplicação (por escalares), com forma $:: k \times A \rightarrow A$. (As notações $\circ \mathrm{e}$ . foram escolhidas apenas para diferenciar as operações aqui. Na prática, ambas são denotadas por justaposição). Além disso, estas três operações devem satisfazer as seguintes propriedades:

1. A tripla $(A,+, \circ)$ é um anel associativo com unidade, isto é, vale o seguinte:

(a) Associatividade da soma:

$$
(a+b)+c=a+(b+c) \text { para todos } a, b, c \in A .
$$

(b) Comutatividade da soma:

$$
a+b=b+a \text { para todos } a, b \in A
$$

(c) Existência de elemento neutro da soma:

Existe $0 \in A$ tal que $a+0=0+a=a$ para todo $a \in A$. 
(d) Existência de elemento oposto da soma:

Para todo $a \in A$ existe $b \in B$ tal que $a+b=b+a=0$.

(e) Distributividade:

$$
a \circ(b+c)=a \circ b+a \circ c \text { e }(a+b) \circ c=a \circ c+b \circ c \text { para todos } a, b, c \in A .
$$

(f) Associatividade da multiplicação:

$$
(a \circ b) \circ c=a \circ(b \circ c) \text { para todos } a, b, c \in A .
$$

(g) Existência de elemento neutro da multiplicação:

Existe $1 \in A$ tal que $a \circ 1=1 \circ a=a$ para todo $a \in A$.

2. A tripla $(A,+,$.$) é um espaço vetorial sobre k$, isto é, além dos axiomas acima, também vale o seguinte:

(a) Distributividade:

$$
\begin{aligned}
& \lambda .(a+b)=\lambda \cdot a+\lambda . b \text { para todos } \lambda \in k \text { e } a, b \in A . \\
& (\lambda+\mu) . a=\lambda . a+\mu . a \text { para todos } \lambda, \mu \in k \text { e } a \in A .
\end{aligned}
$$

(b) Multiplicação pela unidade de $k$ : se 1 denota o elemento unidade de $k$, então:

$$
\text { 1. } a=a \text { para todo } a \in A \text {. }
$$

(c) Homogeneidade:

$$
(\lambda \mu) . a=\lambda .(\mu . a) \text { para todos } \lambda, \mu \in k \text { e } a \in A .
$$

3. As operações o e . são compatíveis, isto é:

$$
\lambda .(a \circ b)=(\lambda . a) \circ b=a \circ(\lambda . b) \text { para todos } \lambda \in k \text { e } a, b \in A .
$$

Observação 2.1.2. Resumidamente, todas as álgebras aqui consideradas serão sobre um corpo, e todas serão associativas e com elemento unidade. O elemento unidade poderá ser denotado tanto como 1 quanto $1_{A}$ se quisermos enfatizar a álgebra $A$.

Além disso, com a intenção de utilizar os Teoremas de Gabriel mais à frente, desde já estaremos sempre assumindo as hipóteses abaixo, caso não haja menção explícita em contrário:

- O corpo $k$ é algebricamente fechado, isto é, todo polinômio com coeficientes em $k$ admite pelo menos uma raiz em $k$.

- $A$ é tem dimensão finita sobre $k$, isto é, a álgebra $A$, quando vista como espaço vetorial sobre $k$, tem dimensão finita. 
Mais à frente (Seção 2.2), quando relembrarmos a definição, também pediremos que as álgebras sejam básicas.

Definição 2.1.3. Sejam $A$ e $B$ duas $k$-álgebras. Uma função $f: A \rightarrow B$ é um homomorfismo de álgebras se $f$ é, simultaneamente, uma transformação $k$-linear e um homomorfismo de anéis que preserva a unidade. Além disso, dizemos que $f$ é um:

- monomorfismo, se $f$ é injetor.

- epimorfismo, se $f$ é sobrejetor.

- isomorfismo, se $f$ é bijetor.

Exemplo 2.1.4. Vamos enumerar alguns exemplos simples de álgebras:

1. O corpo $k$ é uma álgebra sobre si mesmo. Dada uma álgebra $A$ qualquer, $k$ é isomorfo a uma subálgebra de $A$ (isto é, um subconjunto de $A$ que, quando equipado com as operações de $A$ restritas, é uma álgebra por si só), de fato:

$$
k \cong\left\{\lambda .1_{A}: \lambda \in k\right\} \subseteq A
$$

2. A álgebra $M_{n}(k)$, cujos elementos são as matrizes quadradas de ordem $n$ (onde $n \geq 1$ ) com coeficientes em $k$, é uma $k$-álgebra. Existem vários exemplos de álgebras que são subálgebras de $M_{n}(k)$, como por exemplo a $k$-álgebra $T_{n}(k)$ das matrizes quadradas triangulares superiores com ordem $n$ e coeficientes em $k$.

3. Um exemplo famoso é o da álgebra de Kronecker. Esta é definida por:

$$
A=\left(\begin{array}{cc}
k & 0 \\
k^{2} & k
\end{array}\right)=\left\{\left(\begin{array}{cc}
\lambda_{1} & 0 \\
(\mu, \nu) & \lambda_{2}
\end{array}\right): \lambda_{1}, \lambda_{2}, \mu, \nu \in k\right\}
$$

A multiplicação (interna) de dois elementos dessa álgebra é definida naturalmente por:

$$
\left(\begin{array}{cc}
\lambda_{1} & 0 \\
(\mu, \nu) & \lambda_{2}
\end{array}\right)\left(\begin{array}{cc}
\lambda_{1}^{\prime} & 0 \\
\left(\mu^{\prime}, \nu^{\prime}\right) & \lambda_{2}^{\prime}
\end{array}\right)=\left(\begin{array}{cc}
\lambda_{1} \lambda_{1}^{\prime} & 0 \\
\left(\mu \lambda_{1}^{\prime}+\lambda_{2} \mu^{\prime}, \nu \lambda_{1}^{\prime}+\lambda_{2} \nu^{\prime}\right) & \lambda_{2} \lambda_{2}^{\prime}
\end{array}\right)
$$

4. Temos também a $k$-álgebra $k\left[t_{1}, \ldots, t_{n}\right]$ dos polinômios nas variáveis $t_{1}, \ldots, t_{n}$ com coeficientes em $k$. (Neste caso a dimensão da álgebra é infinita).

Vamos agora estabelecer a definição de módulos sobre uma álgebra:

Definição 2.1.5. Seja $A$ uma $k$-álgebra. Um módulo à direita é um conjunto não vazio $M$ equipado com duas operações: uma chamada de soma e denotada por $+: M \times M \rightarrow M$ e outra, chamada de multiplicação (por escalares) e com forma . : $M \times A \rightarrow M$. (Novamente, . é na prática denotada apenas por justaposição). Além disso, estas duas operações devem satisfazer as seguintes propriedades:

1. Associatividade da soma:

$$
(m+n)+p=m+(n+p) \text { para todos } m, n, p \in M .
$$


2. Comutatividade da soma:

$$
m+n=n+m \text { para todos } m, n \in M .
$$

3. Existência de elemento neutro da soma:

Existe $0 \in M$ tal que $m+0=0+m=m$ para todo $m \in M$.

4. Existência de elemento oposto da soma:

Para todo $m \in M$ existe $n \in M$ tal que $m+n=n+m=0$.

5. Distributividade:

$$
\begin{aligned}
& (m+n) \cdot a=m \cdot a+n . a \text { para todos } a \in A \text { e } m, n \in M . \\
& m .(a+b)=m . a+m . b \text { para todos } a, b \in A \text { e } m \in M .
\end{aligned}
$$

6. Multiplicação pela unidade de $A$ : se 1 denota o elemento unidade de $A$, então:

$$
m .1=m \text { para todo } m \in M
$$

7. Homogeneidade:

$$
m \cdot(a b)=(m . a) \cdot b \text { para todos } a, b \in A \text { e } m \in M .
$$

Um $A$-módulo à esquerda é definido de forma dual, sendo que a multiplicação passa a ser da forma . : $A \times M \rightarrow M$.

Observação 2.1.6. Para explicitar que $M$ é um $A$-módulo à direita, usaremos a notação $M_{A}$. Da mesma forma, se for um módulo à esquerda, usaremos a notação ${ }_{A} M$.

Observação 2.1.7. Quando não for explicitado, estaremos sempre assumindo que os módulos são à direita.

Além disso, ainda que módulos que podem ser infinitamente gerados apareçam em alguns momentos, nosso principal interesse é sobre os módulos finitamente gerados. Portanto estaremos sempre assumindo que os módulos são finitamente gerados, exceto quando for explicitado que não precisa haver essa hipótese.

Observação 2.1.8. De forma natural, todo módulo $M$ sobre uma álgebra $A$ é um espaço vetorial sobre $k$ : basta definir, para $\lambda \in k$ e $m \in M, \lambda . m \doteq\left(\lambda .1_{A}\right) . m$. Os axiomas de homogeneidade enunciados acima garantem que as várias estruturas envolvidas são compatíveis. Por exemplo, se $\lambda \in k, a \in A$ e $m \in M$, é direto verificar que $\lambda .(a m)=(\lambda a) . m$.

Note que, como estamos assumindo em geral que as álgebras têm dimensão finita, um módulo sobre uma álgebra é finitamente gerado se e somente se tiver dimensão finita sobre $k$.

Definição 2.1.9. Seja $A$ uma $k$-álgebra, e sejam $M$ e $N$ dois $A$-módulos. Uma função $f: M \rightarrow N$ é um morfismo de módulos se $f$ satisfaz as duas propriedades abaixo: 
1. Para todos $m, n \in M$, vale que $f(m+n)=f(m)+f(n)$.

2. Para todo $m \in M$ e todo $a \in A$, vale que $f(m . a)=f(m) . a$.

Além disso, dizemos que $f$ é um:

- monomorfismo, se $f$ é injetor.

- epimorfismo, se $f$ é sobrejetor.

- isomorfismo, se $f$ é bijetor.

Observação 2.1.10. Seja $A$ uma $k$-álgebra.

- A categoria cujos objetos são módulos à direita sobre $A$ (não necessariamente finitamente gerados) e cujos morfismos são os morfismos de módulos será denotada por $\operatorname{Mod} A$.

- A categoria cujos objetos são módulos à esquerda sobre $A$ (não necessariamente finitamente gerados) e cujos morfismos são os morfismos de módulos será denotada por $A$-Mod.

- A subcategoria plena de $\operatorname{Mod} A$ cujos objetos são os módulos finitamente gerados será denotada por $\bmod A$.

- A subcategoria plena de $A$-Mod cujos objetos são os módulos finitamente gerados será denotada por $A$-mod.

As categorias de módulos têm subobjetos e objetos quocientes, que chamamos de submódulos e módulos quocientes respectivamente. Apenas para relembrar, um submódulo $N$ de um módulo $M$ é um subconjunto de $M$ que, equipado com as operações de $M$ restritas a $N$, é um módulo por si só.

Vamos também relembrar o conceito de álgebra oposta:

Definição 2.1.11. Seja $A$ uma $k$-álgebra, com multiplicação interna denotada por justaposição. A álgebra oposta de $A$, denotada por $A^{o p}$, é uma $k$-álgebra cujo conjunto subjacente é igual ao de $A$, a soma e a multiplicação por escalares são as mesmas de $A$, mas a multiplicação interna é dada pela operação $\circ: A \times A \rightarrow A, \operatorname{com} a \circ b=b a$ para todos $a, b \in A$. (Note que $A$ é comutativa se e somente se $\left.A=A^{o p}\right)$.

A proposição a seguir, de fácil verificação, será usada depois sem necessidade de maiores comentários:

Proposição 2.1.12. Seja $A$ uma $k$-álgebra.

- $\left(A^{o p}\right)^{o p}=A$

- As categorias $A$ - Mod e $\operatorname{Mod} A^{o p}$ são isomorfas.

- Se $A$ é comutativa, as categorias $A$ - Mod e $\operatorname{Mod} A$ são isomorfas.

- As categorias $A$ - $\bmod$ e $\bmod A^{o p}$ são isomorfas.

- Se $A$ é comutativa, as categorias $A-\bmod$ e $\bmod A$ são isomorfas. 
Definição 2.1.13. Sejam $A$ e $B$ duas $k$-álgebras e seja $M$ um conjunto não-vazio. Dizemos que $M$ é um $(A-B)$-bimódulo e usamos a notação ${ }_{A} M_{B}$ para dizer que $M$ é um $A$-módulo à esquerda, um $B$-módulo à esquerda, e que as duas estruturas são compatíveis, isto é, para todos $a \in A, b \in B$ e $m \in M$, vale que $(a m) b=a(m b)$.

\subsection{Noções sobre módulos}

Dedicamos esta seção para concentrar um conteúdo básico de Teoria de Módulos que vai ser citado mais à frente. Para o que segue, estaremos sempre usando a letra $A$ para denotar uma $k$-álgebra.

\section{Espaços Hom e funtores dualidade}

Sejam $M$ e $N$ dois $A$-módulos. O conjunto dos morfismos de módulos da forma $M \rightarrow N$ é denotado por $\operatorname{Hom}_{A}(M, N)$. (Ou por $\operatorname{End}_{A} M$, no caso em que $\left.M=N\right)$. Em geral, $\operatorname{Hom}_{A}(M, N)$ não é um $A$-módulo, mas tem estrutura de $k$-espaço vetorial. No entanto, se $M$ ou $N$ for um bimódulo, então nesse caso $\operatorname{Hom}_{A}(M, N)$ terá estrutura de módulo. Esse é o conteúdo da próxima proposição:

Proposição 2.2.1. Sejam $M$ e $N$ dois $k$-espaços vetoriais, e sejam $A$ e $B$ duas $k$-álgebras.

- Se ${ }_{B} M_{A}$ é um $(B-A)$-bimódulo e $N_{A}$ é um $A$-módulo à direita, então o espaço $\operatorname{Hom}_{A}\left({ }_{B} M_{A}, N_{A}\right)$ é um $B$-módulo à direita. Dado $b \in B$ e $f \in \operatorname{Hom}_{A}(M, N)$, a ação de $b$ em $f$ é dada por:

$$
f . b \in \operatorname{Hom}_{A}(M, N),(f . b)(x)=f(b x) \forall x \in M
$$

- Se ${ }_{A} M_{B}$ é um $(A-B)$-bimódulo e ${ }_{A} N$ é um $A$-módulo à esquerda, então o espaço $\operatorname{Hom}_{A}\left({ }_{A} M_{B},{ }_{A} N\right)$ é um $B$-módulo à esquerda. Dado $b \in B$ e $f \in \operatorname{Hom}_{A}(M, N)$, a ação de $b$ em $f$ é dada por:

$$
b . f \in \operatorname{Hom}_{A}(M, N),(b . f)(x)=f(x b) \forall x \in M
$$

Vamos aplicar a Proposição 2.2.1 no caso particular em que $A$ é o corpo $k$ (que é, trivialmente, uma $k$-álgebra), e em que $B$ é uma álgebra qualquer, que por comodidade vamos denotar pela letra $A$. Se $M_{A}$ é um $A$-módulo à direita, o espaço $\operatorname{Hom}_{k}\left({ }_{k} M_{A, k} k\right)$ é chamado de dual de $M$ e denotado por $D(M)$. Como vimos acima, $D(M)$ é um $A$-módulo à esquerda. Analogamente, se ${ }_{A} M$ é um $A$-módulo à esquerda, então $D(M)=\operatorname{Hom}_{k}\left({ }_{A} M_{k}, k_{k}\right)$ é um $A$-módulo à direita.

Isso mostra que ficam bem definidos dois funtores: um é $\operatorname{Hom}_{k}\left(k^{-},{ }_{k} k\right): \bmod A \rightarrow A$ - mod, e outro é $\operatorname{Hom}_{k}\left(-{ }_{k}, k_{k}\right): A-\bmod \rightarrow \bmod A$. Como ambos levam um objeto $M$ no espaço $\operatorname{Hom}_{k}(M, k)$, eles são denotados pela mesma letra $D$ e chamados de funtor dualidade. Vamos lembrar como $D$ atua nos morfismos. Sejam $M, N$ dois $A$-módulos à direita (ou à esquerda), e seja $f: M \rightarrow N$ um morfismo. Então $D(f)=\operatorname{Hom}_{k}(f, k): \operatorname{Hom}_{k}(N, k) \rightarrow \operatorname{Hom}_{k}(M, k)$ é um morfismo de $A$-módulos à esquerda (ou à direita, respectivamente). Ele é dado por:

$$
\forall g \in \operatorname{Hom}_{k}(N, k), D(f)(g)=g \circ f
$$




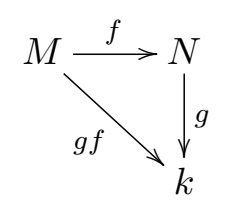

Note que $D$ é um funtor contravariante. Além disso, como estamos considerando módulos com dimensão finita, é um fato bem conhecido de álgebra linear que temos um isomorfismo natural $D \circ D \cong i d$, onde $i d$ é o funtor identidade. Isso mostra que $D$ é uma dualidade entre as categorias $\bmod A$ e $A$-mod, ou seja, $D$ é um funtor contravariante com um quasi-inverso. Isso, por sua vez, implica que $D$ preserva propriedades de módulos, se estas forem auto-duais, ou as antipreserva, se estas tiverem uma propriedade dual distinta da original. Faremos uso deste fato mais à frente.

Antes de passar para o próximo item, vamos usar os funtores Hom para brevemente relembrar o que são módulos projetivos e injetivos.

Definição 2.2.2. Seja $M$ um módulo sobre uma álgebra $A$. Dizemos que $M$ é projetivo se o funtor covariante $\operatorname{Hom}_{A}(M,-)$ é exato (i.e., leva sequências exatas em sequências exatas). De forma dual, dizemos que $M$ é injetivo se o funtor contravariante $\operatorname{Hom}_{A}(-, M)$ é exato.

Proposição 2.2.3. Seja $M$ um módulo sobre uma álgebra $A$. As seguintes condições são equivalentes:

- $M$ é projetivo.

- O funtor covariante $\operatorname{Hom}_{A}(M,-)$ leva epimorfismos em epimorfismos.

- Toda sequência exata da forma $0 \rightarrow X \rightarrow Y \rightarrow M \rightarrow 0$ cinde.

- $M$ é somando direto de algum $A$-módulo livre.

De forma dual (exceto pelo último item), as seguintes condições são equivalentes:

- $M$ é injetivo.

- O funtor contravariante $\operatorname{Hom}_{A}(-, M)$ leva monomorfismos em epimorfismos.

- Toda sequência exata da forma $0 \rightarrow M \rightarrow X \rightarrow Y \rightarrow 0$ cinde.

Para a demonstração da proposição acima, ver [2] ou [14].

\section{Álgebras Tensoriais}

Veremos mais adiante que as álgebras de caminhos generalizadas são casos particulares de álgebras tensoriais, então vamos dedicar um espaço para lembrar este conceito.

Seja $A$ uma $k$-álgebra e ${ }_{A} M_{A}$ um $(A-A)$-bimódulo. A álgebra tensorial $T(A, M)$, como espaço vetorial, é igual à soma direta

$$
T(A, M)=A \oplus M \oplus\left(M \otimes_{A} M\right) \oplus\left(M \otimes_{A} M \otimes_{A} M\right) \oplus \ldots
$$

Como cada somando direto de $T(A, M)$ é um $(A-A)$-bimódulo, também $T(A, M)$ é um $(A-A)$ bimódulo. 
Vejamos como é a multiplicação em $T(A, M)$. Ela é unicamente determinada pelo produto entre tensores elementares, que é dado por: se $a, b \in A, m_{1}, m_{2}, \ldots, m_{t} \in M$ e $1<s<t$, então

$$
\begin{aligned}
a . b & =a b(\text { produto em } A) \\
a .\left(m_{1} \otimes m_{2} \otimes \ldots \otimes m_{t}\right) & =\left(a m_{1}\right) \otimes m_{2} \otimes \ldots \otimes m_{t} \\
\left(m_{1} \otimes \ldots \otimes m_{t-1} \otimes m_{t}\right) . b & =m_{1} \otimes \ldots \otimes m_{t-1} \otimes\left(m_{t} b\right) \\
\left(m_{1} \otimes \ldots \otimes m_{s}\right) .\left(m_{s+1} \otimes \ldots \otimes m_{t}\right) & =m_{1} \otimes \ldots \otimes m_{s} \otimes m_{s+1} \otimes \ldots \otimes m_{t}
\end{aligned}
$$

Note ainda que $A$ é uma subálgebra de $T(A, M)$, e que o elemento identidade $1_{A}$ de $A$ é também o elemento identidade de $T(A, M)$ em relação à multiplicação definida acima.

Um exemplo simples de álgebra tensorial é a álgebra de polinômios a uma variável $t$ com coeficientes em $k$, denotada por $k[t]$. No caso, $k$ faz o papel da álgebra $A$ acima e o módulo $M$, por sua vez, corresponde ao $k$-espaço vetorial tendo o conjunto $\{t\}$ como base.

A propriedade mais importante que vamos utilizar sobre as álgebras tensoriais é a propriedade universal, que será transcrita abaixo. Ela facilita a formalização de ideias, uma vez que pode ser usada para mostrar que certas aplicações tendo uma álgebra tensorial como domínio estão bem definidas.

Proposição 2.2.4 (Propriedade Universal das Álgebras Tensoriais,[4],Lema 1.2). Sejam $A$ e $B$ $k$-álgebras e $M$ um $(A-A)$-bimódulo. Sejam ainda $f_{0}: A \rightarrow B$ um homomorfismo de álgebras e $f_{1}: M \rightarrow B$ um morfismo de $(A-A)$-bimódulos, onde $B$ tem estrutura de $(A-A)$-bimódulo via restrição de escalares por $f_{0}$. Então existe um único homomorfismo de álgebras $f: T(A, M) \rightarrow B$ tal que $\left.f\right|_{A}=f_{0}$ e $\left.f\right|_{M}=f_{1}$.

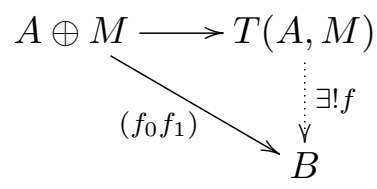

\section{Radical}

Aqui vamos relembrar o conceito de radical de módulos e de álgebras, bem como algumas caracterizações úteis. Existe, na literatura, mais de um tipo de radical, mas aqui o único conceito com o qual vamos trabalhar é o de radical de Jacobson. A abordagem feita aqui se baseia nas referências [2] e [1].

Definição 2.2.5. Seja $A$ uma $k$-álgebra.

- Seja $M_{A}$ um $A$-módulo. O radical de $M$, denotado por $\operatorname{rad} M$, é definido como sendo a intersecção de todos os submódulos maximais de $M$.

- O radical da álgebra $A$, denotado por $\operatorname{rad} A$, é definido como sendo o radical do $A$-módulo regular $A_{A}$, ou seja, é igual à intersecção dos ideais à direita maximais de $A$.

Observação 2.2.6. Como $\operatorname{rad} A$ é intersecção de ideais à direita de $A, \operatorname{rad} A$ é um ideal à direita. Mas na verdade é possível mostrar que $\operatorname{rad} A$ é um ideal bilateral, e que é igual à intersecção dos ideais à esquerda maximais de $A$. 
Proposição 2.2.7. O radical de uma álgebra $A$ é o único ideal bilateral $I$ dessa álgebra tal que $I$ é nilpotente (i.e., existe $n>0$ satisfazendo $I^{n}=0$ ) e tal que a álgebra quociente $A / I$ é semissimples.

Observação 2.2.8. O resultado acima vale não só para álgebras de dimensão finita, mas para qualquer álgebra artiniana.

Definição 2.2.9. Seja $A$ uma álgebra.

- Seja $x \in I$. Dizemos que $x$ é um elemento quasirregular de $A$ se $1-x$ é um elemento invertível de $A$.

- Um subconjunto $S$ de $A$ é dito quasirregular se todo elemento de $S$ é quasirregular.

Observação 2.2.10. Todo elemento $x \in A$ nilpotente é quasirregular. De fato, se $x^{n}=0$, então $(1-x)\left(1+x+\ldots+x^{n-1}\right)=\left(1+x+\ldots+x^{n-1}\right)(1-x)=1$.

Proposição 2.2.11. ([1],15.3) Seja $S$ um subconjunto de uma álgebra $A$. As seguintes afirmações são equivalentes:

1. $S$ é o radical de $A$.

2. $S$ é a união de todos os ideais (à esquerda, à direita, ou bilaterais) quasirregulares de $A$.

3. $S=\{x \in A: r x s$ é quasirregular para todos $r, s \in A\}$

4. $S=\{x \in A: r x$ é quasirregular para todo $r \in A\}$

5. $S=\{x \in A: x s$ é quasirregular para todo $s \in A\}$

\section{Módulos simples e indecomponíveis}

Seja $M$ um $A$-módulo. Dizemos que $A$ é simples se $M$ não possui nenhum submódulo além do módulo nulo e do próprio $M$. O Lema de Schur nos diz que $M$ é simples se e somente se $\operatorname{End}_{A} M$ é uma álgebra de divisão, ou seja, uma álgebra tal que todo elemento não-nulo é inversível.

Dizemos que $M$ é indecomponível se $M$ não pode ser decomposto como soma direta de dois módulos não-nulos. As álgebras com as quais lidamos são artinianas em particular, e neste caso é bem sabido que $M$ é indecomponível se e somente se $\operatorname{End}_{A} M$ é uma álgebra local (isso quer dizer, por exemplo, que rad $A$ é um ideal maximal).

\section{Idempotentes e classificação dos módulos}

Sabemos que, para toda álgebra $A$, existe um subconjunto finito de $A$, denotado por $E=$ $\left\{e_{1}, \ldots, e_{n}\right\}$, satisfazendo as seguintes propriedades:

- Cada $e_{i} \in E$ é idempotente, isto é, $e_{i}^{2}=e_{i}$.

- Os $e_{i}$ 's são dois-a-dois ortogonais, isto é, $e_{i} e_{j}=0$ para cada par de índices $i, j \operatorname{com} i \neq j$.

- Cada $e_{i}$ é primitivo, ou seja, $e_{i}$ não pode ser decomposto como a soma de dois elementos idempotentes ortogonais de $A$.

- $E$ é um conjunto completo, isso quer dizer que $1_{A}=e_{1}+e_{2}+\ldots+e_{n}$. 
Com esta notação, temos o seguinte resultado:

Teorema 2.2.12. Seja $A$ uma álgebra e seja $E$ um conjunto completo de idempotentes primitivos dois-a-dois ortogonais.

- O conjunto $\left\{e_{1} A, \ldots, e_{n} A\right\}$ é um conjunto completo de representantes de classes de isomorfismo de $A$-módulos projetivos indecomponíveis.

- O conjunto $\left\{e_{1} A / e_{1}(\operatorname{rad} A), \ldots, e_{n} A / e_{n}(\operatorname{rad} A)\right\}$ é um conjunto completo de representantes de classes de isomorfismo de $A$-módulos simples.

- O conjunto $\left\{D\left(A e_{1}\right), \ldots, D\left(A e_{n}\right)\right\}$ (onde $D: A-\bmod \rightarrow \bmod A$ é a dualidade) é um conjunto completo de representantes de classes de isomorfismo de $A$-módulos injetivos indecomponíveis.

Definição 2.2.13. Seja $A$ uma álgebra. Dizemos que $A$ é básica se os elementos do conjunto $\left\{e_{1} A, \ldots, e_{n} A\right\}$ (tal como no Teorema 2.2.12 acima) são dois-a-dois não isomorfos entre si.

Proposição 2.2.14. Uma $k$-álgebra $A$ é básica se e somente se a álgebra quociente $A / \operatorname{rad} A$ é isomorfa a um produto direto de cópias de $k$.

Observação 2.2.15. Vale a pena citar que já assumimos no enunciado acima que $k$ é algebricamente fechado. Em geral, vale que uma álgebra $A$ é básica se e somente se $A / \operatorname{rad} A$ é isomorfa a um produto direto de anéis de divisão que contém $k$.

Teorema 2.2.16 (Morita). Seja $A$ uma álgebra de dimensão finita sobre $k$ algebricamente fechado. Então existe uma $k$-álgebra $B$ básica tal que as categorias $\operatorname{Mod} A$ e $\operatorname{Mod} B$ são equivalentes. Tal equivalência se restringe a uma equivalência de categorias $\bmod A \cong \bmod B$.

Observação 2.2.17. Mesmo que uma álgebra não seja básica, pelo Teorema de Morita, a categoria de módulos sobre esta álgebra é equivalente à categoria de módulos de uma outra que é básica. Portanto, sem perda de generalidade, estaremos sempre assumindo, salvo menção em contrário, que as álgebras em questão são básicas.

\section{Grupo de Grothendieck}

Vamos aqui relembrar o conceito de grupo de Grothendieck. Ainda que seja um conceito muito mais amplo, o tratamento feito aqui é apenas ad hoc. Para uma exposição mais detalhada sobre este assunto, recomendamos [3],[2] e [14].

Definição 2.2.18. Para cada $A$-módulo $M$, denotamos por $\tilde{M}$ a classe de isomorfismo de $M$. Considere $\mathcal{F}$, definido como o grupo abeliano livre gerado por todas classes de isomorfismo de $A$ módulos finitamente gerados. Seja $\mathcal{F}^{\prime}$ o subgrupo de $\mathcal{F}$ gerado por todos os elementos da forma $\tilde{L}+\tilde{N}-\tilde{M}$, onde $L, M$ e $N$ são $A$-módulos tais que existe uma sequência exata $0 \rightarrow L \rightarrow M \rightarrow$ $N \rightarrow 0$. Então o grupo de Grothendieck de $A$, denotado por $K_{0}(A)$, é definido como sendo o quociente $\mathcal{F} / \mathcal{F}^{\prime}$.

Teorema 2.2.19. As classes de equivalência das classes de isomorfismo de $A$-módulos simples formam uma $\mathbb{Z}$-base do grupo $K_{0}(A)$. Em particular, todos os conjuntos completos de idempotentes primitivos dois a dois ortogonais em $A$ têm a mesma cardinalidade, que é igual ao posto do grupo $K_{0}(A)$, denotado por rk $K_{0}(A)$. 


\subsection{Aljavas (quivers)}

Nesta seção, vamos recordar o conceito de aljava (chamado de quiver em inglês), que é fundamental na construção tanto das álgebras de caminhos quanto da generalização delas, que são as álgebras de caminhos generalizadas. A grosso modo, uma aljava é um caso particular de um grafo orientado. Vamos fixar as notações por meio da definição abaixo:

Definição 2.3.1. Uma aljava (ou quiver) é uma quádrupla $Q=\left(Q_{0}, Q_{1}, s, e\right)$, na qual $Q_{0}$ e $Q_{1}$ são conjuntos e $s, e: Q_{1} \rightarrow Q_{0}$ são funções. Os elementos de $Q_{0}$ são chamados de vértices, e os elementos de $Q_{1}$ são chamados de flechas. Dada uma flecha $\alpha \in Q_{1}, s(\alpha)$ é chamado de começo de $\alpha$, e $e(\alpha)$ é chamado de término de $\alpha$.

Definição 2.3.2. Seja $Q$ uma aljava.

- Dados dois vértices $i, j \in Q_{0}$, denotamos $Q(i, j)=\left\{\alpha \in Q_{1}: s(\alpha)=i\right.$ and $\left.e(\alpha)=j\right\}$ e também denotamos por $[i, j]_{Q}$ o número de flechas da forma $i \rightarrow j$ que pertencem a $Q_{1}$. (O que significa que $\left.[i, j]_{Q}=|Q(i, j)|\right)$.

- Se, para algum par de vértices $i, j$ de $Q$ valer que $[i, j]>1$, dizemos que existem flechas múltiplas entre $i$ e $j$.

- Se $\alpha$ é uma flecha de $Q$ e $s(\alpha)=e(\alpha)$, então a flecha $\alpha$ é chamada de loop.

Observação 2.3.3. Nós sempre estaremos assumindo aqui que uma dada aljava $Q$ é finita, o que quer dizer que $Q_{0}$ e $Q_{1}$ são conjuntos finitos.

Definição 2.3.4. Dada uma aljava $Q$, definimos a aljava oposta de $Q$, denotada por $Q^{o p}$ : os vértices e as flechas de $Q^{o p}$ são os mesmos de $Q$, mas as flechas têm sentido invertido. Simbolicamente, $\left(Q^{o p}\right)_{0}=Q_{0}$ e para quaisquer dois vértices $i$ e $j, Q^{o p}(i, j)=Q(j, i)$.

Definição 2.3.5. Seja $Q$ uma aljava e seja $i \in Q_{0}$ um vértice de $Q$.

- Dizemos que $i$ é uma fonte de $Q$ se não existe nenhuma flecha de $Q$ terminando em $i$.

- Dizemos que $i$ é um poço de $Q$ se não existe nenhuma flecha de $Q$ começando em $i$.

Definição 2.3.6. Seja $Q$ uma aljava. Um caminho de comprimento 0 sobre $Q$ é simplesmente um vértice de $Q$. Nós distinguimos um vértice $i \in Q_{0}$ de $Q$ do caminho de comprimento zero associado a ele denotando o caminho por $\epsilon_{i}$. Nós também dizemos que $\epsilon_{i}$ começa e termina em $i$ e denotamos $\epsilon_{i}: s(\epsilon)=i \rightsquigarrow e\left(\epsilon_{i}\right)=i$.

Um caminho de comprimento $t$ sobre $Q$ é uma sequência finita $\gamma=\left(\gamma_{1}, \ldots, \gamma_{t}\right)$, onde $t \in \mathbb{N}$ e $\gamma_{1}, \ldots, \gamma_{t} \in Q_{1}$ são flechas de $Q$ tais que para cada $i>1, s\left(\gamma_{i+1}\right)=e\left(\gamma_{i}\right)$. Os vértices $s\left(\gamma_{1}\right)$ e $e\left(\gamma_{t}\right)$, respectivamente, são chamados de começo e término do caminho $\gamma$. Nós ainda usamos a seguinte notação para caminhos: $\gamma=\gamma_{1} \ldots \gamma_{t}: s(\gamma) \rightsquigarrow e(\gamma)$. Quando for necessário distinguir este conceito do conceito de $\mathcal{A}$-caminhos a ser discutido mais à frente, vamos chamar $\gamma$ de caminho ordinário sobre $Q$.

Em qualquer caso, podemos denotar o comprimento de $\gamma$ por $l(\gamma)$.

Além disso, um caminho $\gamma$ é chamado de ciclo orientado se ele começa e termina no mesmo vértice e tem comprimento não-nulo. Se $Q$ não tiver nenhum ciclo orientado, dizemos que $Q$ é uma aljava acíclica. 
Definição 2.3.7. Seja $Q$ uma aljava.

- Um passeio sobre $Q$ entre dois vértices $i, j \in Q_{0}$ é uma sequência finita de flechas $\left(\gamma_{1}, \gamma_{2}, \ldots, \gamma_{t}\right)$ de $Q$, tal que $\gamma_{1}$ começa ou termina em $i, \gamma_{t}$ começa ou termina em $j$, e tal que, para todo $l>1, \gamma_{l-1}$ e $\gamma_{l}$ têm um vértice em comum, o que quer dizer que a intersecção $\left\{s\left(\gamma_{l-1}\right), e\left(\gamma_{l-1}\right)\right\} \cap\left\{s\left(\gamma_{l}\right), e\left(\gamma_{l}\right)\right\}$ é não-vazia.

- Dizemos que a aljava $Q$ é conexa se para todo par de vértices $i, j \in Q_{0}$, existe um passeio sobre $Q$ entre $i$ e $j$.

Definição 2.3.8. Seja $Q=\left(Q_{0}, Q_{1}, s, e\right)$ uma aljava. Uma subaljava de $Q$ é uma aljava $Q^{\prime}=$ $\left(Q_{0}^{\prime}, Q_{1}^{\prime}, s^{\prime}, e^{\prime}\right)$ tal que $Q_{0}^{\prime} \subseteq Q_{0}, Q_{1}^{\prime} \subseteq Q_{1}, s\left(Q_{1}^{\prime}\right) \subseteq Q_{0}^{\prime}, s^{\prime}=\left.s\right|_{Q_{0}^{\prime}}, e\left(Q_{1}^{\prime}\right) \subseteq Q_{0}^{\prime}$, e $e^{\prime}=\left.e\right|_{Q_{0}^{\prime}}$. Dizemos que $Q^{\prime}$ é plena em $Q$ se para todo par de vértices $i, j \in Q_{0}^{\prime}, Q^{\prime}(i, j)=Q(i, j)$.

Exemplo 2.3.9. Vamos dar um exemplo de aljava para ilustrar as definições desta seção. Seja $Q=\left(Q_{0}, Q_{1}, s, e\right)$ uma aljava, onde $Q_{0}=\{1,2,3,4\}, Q_{1}=\{\alpha, \beta, \gamma, \delta\}, s(\alpha)=1, e(\alpha)=2$, $s(\beta)=2, e(\beta)=3, s(\gamma)=3, e(\gamma)=2, s(\delta)=1$ e $e(\delta)=1$. Essa é uma descrição abstrata da aljava $Q$, mas na prática uma aljava é descrita usando um diagrama tal como o que segue:

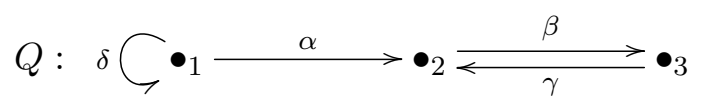

Nesta aljava, por exemplo, $[1,1]=1,[1,2]=1,[1,3]=0$. Não há flechas múltiplas em $Q$, mas a flecha $\delta$ é um loop sobre o vértice 1 . O vértice 4 é a única fonte e o único poço da aljava. Alguns exemplos de caminhos sobre $Q$ incluem $\alpha, \delta \alpha, \alpha \beta, \beta \gamma$. Além disso, a aljava não é acíclica porque tem ciclos orientados, como por exemplo $\beta \gamma, \gamma \beta$ ou $\delta^{n}$, onde $n$ é um número natural. A aljava também não é conexa porque não há nenhum passeio que ligue 4 aos outros vértices. Um exemplo de subaljava de $Q$ é a aljava $Q^{\prime}$ abaixo:
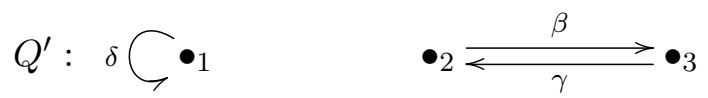

Note no entanto que $Q^{\prime}$ não é plena em $Q$. Por outro lado, a subaljava plena determinada pelos vértices 1,2 e 3 é conexa. Por fim, a aljava oposta de $Q$ é dada por:

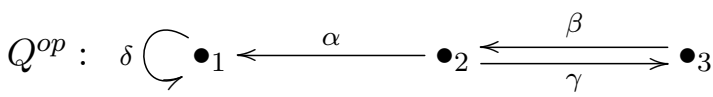

\subsection{A categoria das aljavas}

Complementando a Seção 2.3, vamos fazer algumas considerações sobre a categoria das aljavas. Primeiro, vamos estabelecer sua definição formal.

Definição 2.4.1. A categoria Quiv é definida pelas seguintes informações:

- A classe de objetos de Quiv é a classe de todas as aljavas. 
- Dadas duas aljavas $Q=\left(Q_{0}, Q_{1}, s, e\right)$ e $Q^{\prime}=\left(Q_{0}^{\prime}, Q_{1}^{\prime}, s, e\right)$, um morfismo de aljavas $f$ : $Q \rightarrow Q^{\prime}$ é dado por um par $f=\left(f_{0}, f_{1}\right)$, onde $f_{0}: Q_{0} \rightarrow Q_{0}^{\prime}$ e $f_{1}: Q_{1} \rightarrow Q_{1}^{\prime}$ são funções satisfazendo $s f_{1}=f_{0} s$ e $e f_{1}=f_{0} e$, ou seja, o seguinte diagrama é comutativo:

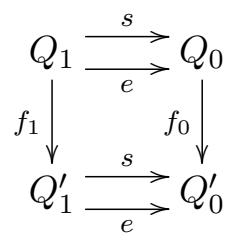

Observação 2.4.2. Note que, se $f_{0}$ e $f_{1}$ são sobrejetores (ou injetores), então $f$ é um epimorfismo no sentido categórico (ou monomorfismo, respectivamente).

É fácil ver que a categoria Quiv possui subobjetos, estes são simplesmente as subaljavas já discutidas na Seção 2.3. Nosso interesse aqui no entanto é discutir o conceito dual, que é de objetos quocientes.

Definição 2.4.3. Seja $Q$ uma aljava e seja $\sim \subseteq Q_{0} \times Q_{0}$ uma relação de equivalência sobre os vértices de $Q$. Definimos a aljava quociente $\bar{Q}=\frac{Q}{\sim}$. O conjunto de vértices de $\bar{Q}$ é o conjunto quociente $\frac{Q_{0}}{\sim}$, e dados dois vértices $\bar{a}, \bar{b} \in \bar{Q}_{0}$, o número de flechas que começam em $\bar{a}$ e terminam em $\bar{b}$ é dado pela seguinte fórmula:

$$
[\bar{a}, \bar{b}]_{\bar{Q}}=\max \left\{[i, j]_{Q}: i \in \bar{a}, j \in \bar{b}\right\}
$$

Usando a Observação 2.4.2 acima, é direto verificar que existe um epimorfismo de aljavas $\pi=$ $\left(\pi_{0}, \pi_{1}\right): Q \rightarrow \bar{Q}$, onde $\pi_{0}: Q_{0} \rightarrow \frac{Q_{0}}{\sim}$ é a projeção canônica no quociente e para todo par de vértices $i, j \in Q_{0}, \pi_{1}$ induz uma função injetora $Q(i, j) \rightarrow \bar{Q}(\bar{i}, \bar{j})$. O epimorfismo $\pi$, no entanto, não precisa ser único.

A proposição a seguir é de fácil verificação:

Proposição 2.4.4. Sejam $Q$ uma aljava e $\sim$ uma relação de equivalência em $Q_{0}$. Considerando a aljava quociente $\bar{Q}=\frac{Q}{\sim}$ e denotando a classe de equivalência de um vértice $i$ por $\bar{i}$, valem as seguintes propriedades:

1. Para quaisquer dois vértices $i, j \in Q_{0}$, vale que $[i, j]_{Q} \leq[\bar{i}, \bar{j}]_{\bar{Q}}$. Em particular:

(a) Se $Q$ tem setas múltiplas, então $\bar{Q}$ também terá.

(b) Se $i \sim j$ e existe uma flecha da forma $i \rightarrow j$, então haverá em $\bar{Q}$ um loop sobre $\bar{i}$.

2. Se $Q$ é conexa, então $\bar{Q}$ também será.

Exemplo 2.4.5. Vamos dar um exemplo de aljava quociente. Considere a aljava $Q$ abaixo: 


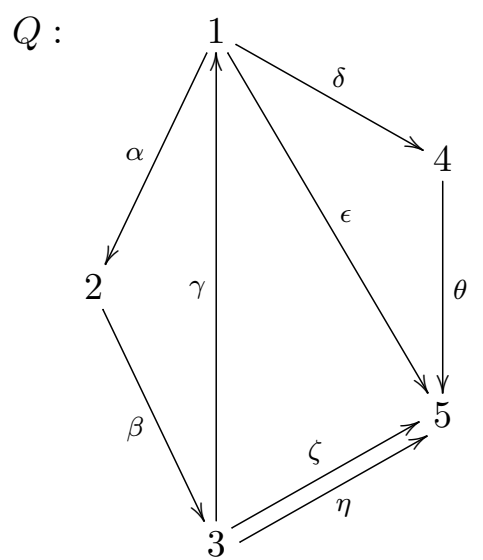

Seja $\sim \subseteq Q_{0} \times Q_{0}$ a menor relação de equivalência que satisfaz $1 \sim 2 \sim 3$ e $4 \sim 5$. Então a aljava quociente $\bar{Q}$ é dada por:

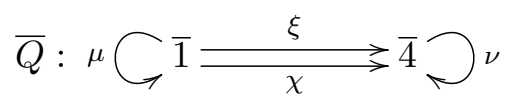

Um possível epimorfismo de aljavas $\pi=\left(\pi_{0}, \pi_{1}\right): Q \rightarrow \bar{Q}$ é o seguinte:

$$
\begin{aligned}
& \pi_{0}(1)=\pi_{0}(2)=\pi_{0}(3)=\overline{1}, \quad \pi_{0}(4)=\pi_{0}(5)=\overline{4} \\
& \pi_{1}(\alpha)=\pi_{1}(\beta)=\pi_{1}(\gamma)=\mu, \pi_{1}(\theta)=\nu \\
& \pi_{1}(\delta)=\pi_{1}(\epsilon)=\pi_{1}(\zeta)=\xi, \pi_{1}(\eta)=\chi
\end{aligned}
$$

\section{5 Álgebras de Caminhos}

Vamos aqui relembrar o conceito original de álgebra de caminhos, tendo em vista que o conceito generalizado será assunto principal mais à frente.

Seja $Q$ uma aljava. Denote por $k Q$ o $k$-espaço vetorial tendo como base o conjunto de todos os caminhos sobre $Q$. Queremos definir uma multiplicação interna em $k Q$. Por linearidade, é suficiente definir o que significa a multiplicação de dois caminhos sobre $Q$. Esta, por sua vez, é dada pela composição desses caminhos, sendo esta definida naturalmente, usando a ideia de justaposição. Vamos dar mais detalhes: seja $\epsilon_{i}$ o caminho de comprimento 0 sobre um vértice $i \in Q_{0}$, e seja $\gamma$ um caminho qualquer sobre $Q$. Então $\epsilon_{i} \gamma$ é definido como $\gamma$ se $s(\gamma)=i$, e definido como zero caso contrário. Analogamente, $\gamma \epsilon_{i}$ é definido como $\gamma$ se $e(\gamma)=i$, e definido como zero caso contrário. Além disso, sejam $\gamma=\gamma_{1} \ldots \gamma_{t}$ e $\delta=\delta_{1} \ldots \delta_{s}$ dois caminhos sobre $Q$. Então $\gamma \delta$ é definido como o caminho $\gamma_{1} \ldots \gamma_{t} \delta_{1} \ldots \delta_{s}$ se $e(\gamma)=s(\delta)$, e definido como zero caso contrário.

Com essa multiplicação, $k Q$ é uma $k$-álgebra, chamada de álgebra de caminhos sobre o quiver Q. Vamos abreviar álgebra de caminhos por $\mathbf{A C}$.

Quando for necessário distinguir o conceito de álgebra de caminhos desta seção do conceito de álgebras de caminhos generalizadas a ser discutido posteriormente, vamos dizer que $k Q$ é a álgebra de caminhos ordinária sobre $Q$.

Observação 2.5.1. É fácil se convencer de que a composição de caminhos é associativa, e que portanto $k Q$ é uma álgebra associativa. Além disso, como estamos sempre assumindo que $Q$ é uma 
aljava finita, $k Q$ tem um elemento identidade, dado por

$$
1_{k Q}=\sum_{i \in Q_{0}} \epsilon_{i}
$$

Proposição 2.5.2. Seja $Q$ uma aljava. Então $k Q$ tem dimensão finita se e somente se $Q$ é uma aljava acíclica.

Demonstração. Suponha que $Q$ não é acíclica. Então $Q$ possui um ciclo orientado, que denotaremos por $\gamma$. Daí $k Q$ tem dimensão infinita porque possui um conjunto linearmente independente infinito, que é $\left\{\gamma, \gamma^{2}, \gamma^{3}, \ldots\right\}$.

Reciprocamente, se $Q$ é acíclica, então, como é finita, existe um número finito de caminhos sobre $Q$, e logo a base canônica de $k Q$ formada pelos caminhos é finita, ou seja, $k Q$ tem dimensão finita.

Como as flechas de $Q$ são caminhos de comprimento 1, e portanto podem ser vistas como elementos de $k Q$, faz sentido considerar o ideal de $k Q$ gerado pelas flechas de $Q$ : denotamos este ideal por $J$.

Proposição 2.5.3. Se uma aljava $Q$ é acíclica, $J$ coincide com o radical de Jacobson da álgebra de caminhos $k Q$.

Demonstração. Se $Q$ é acíclica, pela Proposição 2.5.2, $k Q$ tem dimensão finita, logo podemos usar a Proposição 2.2.7. Portanto, é suficiente provar que $J$ é um ideal nilpotente e que $k Q / J$ é uma álgebra semissimples.

Como $Q$ é finita e acíclica, existe um número finito de caminhos sobre $Q$, e em particular existe um número natural $n$ tal que nenhum caminho sobre $Q$ tem comprimento maior do que $n$. Portanto $J^{n}=0$, mostrando que o ideal $J$ é nilpotente.

A álgebra $k Q / J$ é isomorfa à álgebra produto $\prod_{i \in Q_{0}} k \epsilon_{i}$, que por sua vez é isomorfa ao produto de cópias de $k, \prod_{i \in Q_{0}} k$, que é semissimples pelo Teorema de Wedderburn-Artin. Logo $k Q / J$ é semissimples e isso conclui a demonstração.

Observação 2.5.4. Veremos mais à frente (Teorema 2.6.1) que, apesar de nem toda álgebra ser isomorfa a uma álgebra de caminhos, toda álgebra é isomorfa a um quociente de uma álgebra de caminhos. No entanto, não estamos interessados em identificar vértices ou flechas com o elemento zero no quociente, mas como o quociente deve ter dimensão finita, é interessante garantir que caminhos suficientemente grandes (incluindo ciclos orientados a partir de determinada potência) estão identificados com o zero. Isso é o que motiva a definição a seguir.

Definição 2.5.5. Seja $I$ um ideal de $k Q$. Este ideal é chamado de admissível se existir um número natural $n$ tal que $J^{n} \subseteq I \subseteq J^{2}$.

Outro conceito importante que será usado aqui será o de relações sobre uma aljava.

Definição 2.5.6. Dada uma aljava $Q$, uma relação (ordinária ou usual) sobre $Q$ é uma combinação $k$-linear de caminhos sobre $Q$, todos tendo comprimento maior ou igual a 2, e todos começando e terminando no mesmo vértice. 
Observação 2.5.7. É um resultado básico que todo ideal admissível de $k Q$ é gerado por um conjunto finito de relações. E reciprocamente, se $Q$ é acíclica, todo conjunto finito de relações gera um ideal admissível de $k Q$.

Observação 2.5.8. Em situações práticas, é costume definir uma álgebra $A$ dando uma aljava $Q$ e um conjunto finito de relações $R$ sobre $Q$. Ao fazer isso, queremos dizer que $A$ é definida por $A=k Q /(R)$, onde $(R)$ é o ideal gerado por $R$. Também pode-se dizer que $A$ é a álgebra de caminhos sobre $Q$ com relações $R$. Aqui vamos abreviar o termo álgebra de caminhos com relações pela sigla ACR.

Também se costuma fazer um abuso de notação e denotar pela mesma letra ( $R$, por exemplo) um conjunto de relações e o ideal gerado por esse conjunto, já que tanto as relações quanto aquilo que elas geram é anulado no quociente.

Exemplo 2.5.9. Vamos exemplificar a discussão da Observação 2.5.8 acima. Definimos uma álgebra $A$ através da seguinte aljava com relações:

$$
Q: 1<\alpha<\beta \quad \beta \alpha=0
$$

Isto significa que $A \doteq k Q / R$, onde $Q$ é aljava definida acima e $R$ é o ideal de $k Q$ gerado pelo elemento $\beta \alpha$, que é uma relação sobre $Q$.

Como $\beta \alpha$ tem comprimento dois, vale que $R \doteq(\beta \alpha) \subseteq J^{2}$. Como nenhum caminho sobre $Q$ tem comprimento maior do que 2 , temos que $J^{3}=0$. Portanto $J^{3} \subseteq R \subseteq J^{2}$ e que $R$ é um ideal admissível de $k Q$.

\subsection{Os Teoremas de Gabriel}

Nesta seção vamos lembrar as versões originais de resultados introduzidos por Gabriel, e que tratam do conceito de álgebras de caminhos. Mais à frente (Teoremas 4.2.3,4.2.5 e 5.1.3), discutiremos versões generalizadas desses resultados, que tratam de álgebras de caminhos generalizadas.

\section{A aljava de Gabriel de uma álgebra}

O primeiro teorema diz respeito ao problema de associar uma aljava $Q_{A}$ a uma álgebra $A$ dada, de maneira tal que $A$ seja o quociente da álgebra de caminhos sobre $Q_{A}$ por um ideal admissível. Formalmente, temos o seguinte enunciado:

Teorema 2.6.1 (P. Gabriel). Seja $A$ uma álgebra (de dimensão finita e básica sobre um corpo algebricamente fechado $k$ ). Então existe uma aljava $Q_{A}$ e um ideal admissível $I$ de $k Q_{A}$ tal que $A \cong k Q_{A} / I$. Além disso, $Q_{A}$ é unicamente determinada por $A$. Se, ainda, $A$ é uma álgebra conexa, então a aljava $Q$ também será conexa.

A aljava $Q_{A}$ é chamada de aljava de Gabriel ou aljava ordinária de $A$. Outra maneira de interpretar o Teorema 2.6.1 é dizer que toda álgebra é, a menos de isomorfismo, uma álgebra de caminhos com relações.

Observação 2.6.2. Não recordaremos aqui toda a demonstração do Teorema 2.6 .1 (ver [3], por exemplo, para uma demonstração completa), mas gostaríamos de recordar alguns argumentos, mais 
especificamente, como a aljava $Q_{A}$ é construída. Seja $E=\left\{e_{1}, \ldots, e_{n}\right\}$ um conjunto de elementos idempotentes primitivos e ortogonais dois-a-dois em $A$. O conjunto de vértices de $Q_{A}$ será então $E$. Além disso, se $e_{i}, e_{j} \in E$, o número de flechas em $Q_{A}$ da forma $e_{i} \rightarrow e_{j}$ é igual ao número natural $\operatorname{dim}_{\mathrm{k}} \frac{e_{i}(\operatorname{rad} A) e_{j}}{e_{i}\left(\operatorname{rad}^{2} A\right) e_{j}}$. Apesar da definição da aljava $Q_{A}$ aparentemente depender da escolha do conjunto $E$, é possível mostrar que qualquer outra escolha resultaria em uma aljava que é simplesmente isomorfa (como aljava) à aljava $Q_{A}$.

Exemplo 2.6.3. Vamos ilustrar a ideia descrita na Observação 2.6 .2 acima com um exemplo prático. Seja $A$ a álgebra descrita abaixo, que é uma subálgebra da álgebra de matrizes $M_{3}(k)$ :

$$
A \doteq\left(\begin{array}{ccc}
k & 0 & 0 \\
k & k & 0 \\
0 & k & k
\end{array}\right) \doteq\left\{\left(\begin{array}{ccc}
a_{11} & 0 & 0 \\
a_{21} & a_{22} & 0 \\
0 & a_{32} & a_{33}
\end{array}\right): a_{11}, a_{21}, a_{22}, a_{32}, a_{33} \in k\right\}
$$

É fácil constatar que o seguinte conjunto é um conjunto completo de idempotentes primitivos dois-a-dois ortogonais em $A$ :

$$
E=\left\{e_{1}=\left(\begin{array}{lll}
1 & 0 & 0 \\
0 & 0 & 0 \\
0 & 0 & 0
\end{array}\right), e_{2}=\left(\begin{array}{lll}
0 & 0 & 0 \\
0 & 1 & 0 \\
0 & 0 & 0
\end{array}\right), e_{3}=\left(\begin{array}{lll}
0 & 0 & 0 \\
0 & 0 & 0 \\
0 & 0 & 1
\end{array}\right)\right\}
$$

Seja $Q_{A}$ a aljava de Gabriel de $A$. Pela Observação 2.6.2, sabemos que $\left(Q_{A}\right)_{0}=E$ e que portanto $Q_{A}$ tem três vértices: $e_{1}, e_{2}$ e $e_{3}$. Vamos calcular as flechas entre estes vértices.

Usando a Proposição 2.2.7, pode-se provar que:

$$
\operatorname{rad} A=\left(\begin{array}{ccc}
0 & 0 & 0 \\
k & 0 & 0 \\
0 & k & 0
\end{array}\right)
$$

e que portanto:

$$
\operatorname{rad}^{2} A=\left(\begin{array}{lll}
0 & 0 & 0 \\
k & 0 & 0 \\
0 & k & 0
\end{array}\right)\left(\begin{array}{lll}
0 & 0 & 0 \\
k & 0 & 0 \\
0 & k & 0
\end{array}\right)=\left(\begin{array}{lll}
0 & 0 & 0 \\
0 & 0 & 0 \\
k & 0 & 0
\end{array}\right)
$$

Para calcular o número de flechas da forma $e_{2} \rightarrow e_{1}$ em $Q_{A}$, observamos que

$$
e_{2}(\operatorname{rad} A) e_{1}=\left(\begin{array}{ccc}
0 & 0 & 0 \\
k & 0 & 0 \\
0 & 0 & 0
\end{array}\right), e_{2}\left(\operatorname{rad}^{2} A\right) e_{1}=0
$$

e que portanto $\left[e_{2}, e_{1}\right]=\operatorname{dim}_{k} \frac{e_{2}(\operatorname{rad} A) e_{1}}{e_{2}\left(\operatorname{rad}^{2} A\right) e_{1}}=1$. Da mesma forma podemos ver que $\left[e_{3}, e_{2}\right]=1 \mathrm{e}$ que não há outras flechas além destas. Juntando as informações, temos que a aljava de Gabriel de $A$ é dada por:

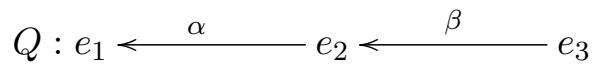

Uma base para $k Q_{A}$ é $\left\{e_{1}, e_{2}, e_{3}, \alpha, \beta, \beta \alpha\right\}$. Podemos então considerar a transformação linear $\phi: k Q_{A} \rightarrow A$ determinada pelas informações abaixo: 


$$
\phi\left(e_{1}\right)=e_{1}, \phi\left(e_{2}\right)=e_{2}, \phi\left(e_{3}\right)=e_{3}, \phi(\alpha)=\left(\begin{array}{lll}
0 & 0 & 0 \\
1 & 0 & 0 \\
0 & 0 & 0
\end{array}\right), \phi(\beta)=\left(\begin{array}{lll}
0 & 0 & 0 \\
0 & 0 & 0 \\
0 & 1 & 0
\end{array}\right), \phi(\beta \alpha)=0
$$

Pode-se provar (isso é feito em geral na demonstração do Teorema de Gabriel) que $\phi$ é, na verdade, um homomorfismo de álgebras sobrejetor. O núcleo de $\phi$ é o subespaço $R$ gerado por $\beta \alpha$, e portanto $R$ é também um ideal de $k Q_{A}$. Portanto $A \cong k Q_{A} / R$ coincide com a álgebra definida no Exemplo 2.5.9.

\section{Representações sobre uma aljava}

O primeiro Teorema de Gabriel diz que álgebras podem ser interpretadas como álgebras de caminhos. O segundo Teorema diz que também os módulos admitem uma interpretação diagramática, desta vez na forma de representações de uma aljava.

Vamos então recordar este conceito na definição abaixo:

Definição 2.6.4. Seja $Q$ uma aljava, e seja $R$ um conjunto de relações sobre $Q$.

- Uma $Q$-representação é um par

$$
M=\left(\left(M_{i}\right)_{i \in Q_{0}},\left(M_{\alpha}\right)_{\alpha \in Q_{1}}\right)
$$

Para cada vértice $i \in Q_{0}, M_{i}$ é um $k$-espaço vetorial, e para cada flecha $\alpha \in \Gamma_{1}, M_{\alpha}: M_{s(\alpha)} \rightarrow$ $M_{e(\alpha)}$ é uma transformação $k$-linear. Além disso, dizemos que $M$ é finitamente gerada se $M_{i}$ tem dimensão finita para cada $i \in \Gamma_{0}$.

- Se $M$ é uma representação tal como no item anterior e $\gamma=\sum_{i=1}^{t} \lambda_{i} \alpha_{i 1} \alpha_{i 2} \ldots \alpha_{i n_{i}}$, onde $\lambda_{i} \in k, \alpha_{i j} \in Q_{1}$, é uma combinação linear de caminhos sobre $Q$ que começam e terminam no mesmo vértice, definimos a transformação linear

$$
\begin{aligned}
& M_{\gamma}: M_{s\left(\alpha_{11}\right)} \rightarrow M_{e\left(\alpha_{1 n_{1}}\right)} \\
& M_{\gamma}=\sum_{i=1}^{t} \lambda_{i} M_{\alpha_{i 1}} M_{\alpha_{i 2}} \ldots M_{\alpha_{i n_{i}}}
\end{aligned}
$$

- Com a notação acima, dizemos que $M$ satisfaz as relações de $R$ se $M_{\gamma}=0$ para toda relação $\gamma \in R$.

- Sejam $M=\left(\left(M_{i}\right)_{i \in Q_{0}},\left(M_{\alpha}\right)_{\alpha \in Q_{1}}\right)$ e $N=\left(\left(N_{i}\right)_{i \in Q_{0}},\left(N_{\alpha}\right)_{\alpha \in Q_{1}}\right)$ duas $Q$-representações. Um morfismo de representações $f: M \rightarrow N$ é uma tupla $f=\left(f_{i}\right)_{i \in Q_{0}}$, tal que, para cada $i \in Q_{0}, f_{i}: M_{i} \rightarrow N_{i}$ é uma transformação $k$-linear, e tal que, para cada flecha $\alpha: i \rightarrow j \in Q_{1}$, vale que $f_{j} M_{\alpha}=N_{\alpha} f_{i}$, ou seja, o seguinte diagrama comuta: 


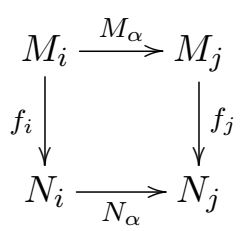

- A categoria das $Q$-representações que satisfazem $R$ é denotada por $\operatorname{Rep}_{k}(Q, R$ ) (ou por $\operatorname{Rep}_{k} Q$, se $R$ é vazio). A subcategoria plena das representações finitamente geradas é denotada por $\operatorname{rep}_{k}(Q, R)$ (ou por $\operatorname{rep}_{k} Q$, se $R$ é vazio).

Teorema 2.6.5 (P. Gabriel). Sejam $Q$ uma aljava e $R$ um conjunto de relações sobre $Q$. Existe uma equivalência de categorias

$$
\operatorname{Rep}_{k}(Q, R) \cong \operatorname{Mod} k Q / R
$$

entre $Q$-representações que satisfazem $R$ e $k Q / R$-módulos. Além disso, esta equivalência se restringe a uma equivalência de categorias

$$
\operatorname{rep}_{k}(Q, R) \cong \bmod k Q / R
$$

entre $Q$-representações finitamente geradas que satisfazem $R$ e $k Q / R$-módulos finitamente gerados.

Não vamos discutir a demonstração do Teorema 2.6.5 neste momento, já que vamos provar uma versão generalizada mais à frente.

Exemplo 2.6.6. Vamos retomar o Exemplo 2.5.9 acima. Consideramos a representação $M$ sobre o par $(Q, R)$ dada por $M \doteq\left(\left\{M_{1}, M_{2}, M_{3}\right\},\left\{M_{\alpha}, M_{\beta}\right\}\right)$, onde $M_{1}=0, M_{2}=M_{3}=k, M_{\alpha}=0$ e $M_{\beta}=i d: k \rightarrow k$. É mais comum descrever uma representação de forma diagrámatica, tal como segue abaixo:

$$
M: \quad 0<0<i d \quad k
$$

Como $M_{\beta} \circ M_{\alpha}=0$, a representação $M$ de fato satisfaz a relação $\beta \alpha$ e portanto é um objeto $\mathrm{em} \operatorname{rep}_{k}(Q, R)$.

Usando a demonstração do Teorema 2.6.5, é possível provar que $M$ é, na verdade, a representação que corresponde ao módulo projetivo indecomponível dado pelo elemento idempotente $\overline{\epsilon_{3}} \in k Q / R$, que é a classe de equivalência do caminho de comprimento zero sobre o vértice 3 . 


\section{Capítulo 3}

\section{Álgebras de Caminhos Generalizadas (ACG)}

\subsection{Definições}

O conceito de álgebras de caminhos generalizadas com o qual vamos trabalhar aqui é o introduzido por F. U. Coelho e S. X. Liu em 2000 ([7]). Além de definir estas álgebras e estabelecer algumas propriedades básicas, o interesse daqueles autores também incluia estudar algumas propriedades sobre anéis, por exemplo, analisar quando uma álgebra de caminhos generalizada é um anel noetheriano ou primo. Eles também provaram alguns resultados sobre unicidade, isto é, sobre o que se pode dizer quando duas álgebras desse tipo são isomorfas.

Definição 3.1.1. Sejam $\Gamma=\left(\Gamma_{0}, \Gamma_{1}, s, e\right)$ uma aljava, e $\mathcal{A}=\left(A_{i}\right)_{i \in \Gamma_{0}}$ uma família de $k$-álgebras, uma para cada vértice de $\Gamma$.

- Um $\mathcal{A}$-caminho de comprimento 0 sobre $\Gamma$ é um elemento do conjunto $\bigcup_{i \in \Gamma_{0}} A_{i}$.

- Para $n>0$, um $\mathcal{A}$-caminho de comprimento $n$ sobre $\Gamma$ é uma sequência da forma

$$
a_{1} \beta_{1} a_{2} \ldots a_{n} \beta_{n} a_{n+1}
$$

onde $\beta_{1} \ldots \beta_{n}$ é um caminho ordinário sobre $\Gamma, a_{i} \in A_{s\left(\beta_{i}\right)}$ se $i \leq n$, e $a_{n+1} \in A_{e\left(\beta_{n}\right)}$.

- Denotamos por $k[\Gamma, \mathcal{A}]$ o $k$-espaço vetorial gerado por todos os $\mathcal{A}$-caminhos sobre $\Gamma$.

- A álgebra de caminhos generalizada (ACG) sobre $\Gamma$ e $\mathcal{A}$ é o espaço vetorial quociente $k(\Gamma, \mathcal{A})=k[\Gamma, \mathcal{A}] / M$, onde $M$ é o subespaço gerado por todos os elementos da forma

$$
a_{1} \beta_{1} \ldots \beta_{j-1}\left(a_{j}^{1}+\ldots+a_{j}^{m}\right) \beta_{j} a_{j+1} \ldots \beta_{n} a_{n+1}-\sum_{l=1}^{m}\left(a_{1} \beta_{1} \ldots \beta_{j-1} a_{j}^{l} \beta_{j} \ldots \beta_{n} a_{n+1}\right)
$$

ou, para $\lambda \in k$,

$$
a_{1} \beta_{1} \ldots \beta_{j-1}\left(\lambda a_{j}\right) \beta_{j} a_{j+1} \ldots \beta_{n} a_{n+1}-\lambda .\left(a_{1} \beta_{1} \ldots \beta_{j-1} a_{j} \beta_{j} a_{j+1} \ldots \beta_{n} a_{n+1}\right)
$$


- A multiplicação em $k(\Gamma, \mathcal{A})$ é induzida pela multiplicação interna dos $A_{i}$ 's e por composição de caminhos. De forma mais detalhada, a multiplicação é definida por linearidade e a partir das seguintes regras:

$$
\left(a_{1} \beta_{1} \ldots \beta_{n} a_{n+1}\right)\left(b_{1} \gamma_{1} \ldots \gamma_{m} b_{m+1}\right)=a_{1} \beta_{1} \ldots \beta_{n}\left(a_{n+1} b_{1}\right) \gamma_{1} \ldots \gamma_{m} b_{m+1}
$$

se $e\left(\beta_{n}\right)=s\left(\gamma_{1}\right)$, e

$$
\left(a_{1} \beta_{1} \ldots \beta_{n} a_{n+1}\right)\left(b_{1} \gamma_{1} \ldots \gamma_{m} b_{m+1}\right)=0
$$

caso contrário.

O comprimento de um $\mathcal{A}$-caminho $\gamma \in k(\Gamma, \mathcal{A})$ será sempre denotado por $l(\gamma)$. Claramente, este sempre é um número inteiro positivo ou nulo.

Exemplo 3.1.2. Este é o exemplo inicial do artigo [7]. Considere a ACG

$$
k<\quad k[t]<\quad \beta \quad k
$$

onde $k[t]$ é a álgebra de polinômios em uma variável, que é $t$. Alguns elementos dessa álgebra são: $t, t^{2}, t^{3}, \ldots, \alpha, t \alpha, t^{2} \alpha, \ldots, \beta, \beta t, \beta t^{2}, \ldots, \beta \alpha, \beta t \alpha, \beta t^{2} \alpha$ e assim por diante. Na verdade, se juntarmos a essa lista as unidades das duas álgebras $k$ que aparecem nas extremidades da aljava, teremos que a álgebra é gerada como espaço vetorial por esses elementos. É direto verificar que esta ACG é isomorfa à algebra de caminhos sobre a seguinte aljava:

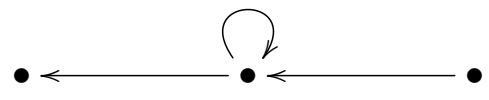

Exemplo 3.1.3. Seja $T_{2}(k)=\left(\begin{array}{ll}k & k \\ 0 & k\end{array}\right)$ a álgebra das matrizes triangulares superiores de ordem 2 com coeficientes em $k$. Considere a ACG

$$
T_{2}(k) \stackrel{\alpha}{\longrightarrow} k
$$

Desta vez, um conjunto de geradores para a álgebra como espaço vetorial é dado por:

$$
\left\{\left(\begin{array}{ll}
1 & 0 \\
0 & 0
\end{array}\right),\left(\begin{array}{ll}
0 & 1 \\
0 & 0
\end{array}\right),\left(\begin{array}{ll}
0 & 0 \\
0 & 1
\end{array}\right),\left(\begin{array}{ll}
1 & 0 \\
0 & 0
\end{array}\right) \alpha,\left(\begin{array}{ll}
0 & 1 \\
0 & 0
\end{array}\right) \alpha,\left(\begin{array}{ll}
0 & 0 \\
0 & 1
\end{array}\right) \alpha, 1_{k}\right\}
$$

Onde $1_{k}$ é a unidade da álgebra igual a $k$ que aparece no vértice em que $\alpha$ termina. Note que $\alpha$ não precisou aparecer entre os elementos acima porque, nesta ACG:

$$
\alpha=1_{T_{2}(k)} \alpha=\left(\begin{array}{ll}
1 & 0 \\
0 & 1
\end{array}\right) \alpha=\left(\begin{array}{ll}
1 & 0 \\
0 & 0
\end{array}\right) \alpha+\left(\begin{array}{ll}
0 & 0 \\
0 & 1
\end{array}\right) \alpha
$$

Além disso, esta ACG é isomorfa à álgebra de caminhos sobre a seguinte aljava: 


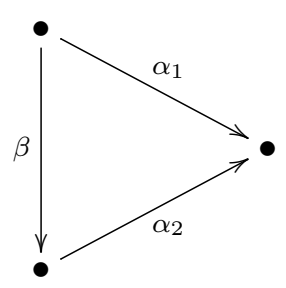

Para provar isso, é possível construir um isomorfismo explícito entre as duas álgebras, porém veremos mais à frente (Teorema 4.2.3) como provar de maneira mais fácil.

Definição 3.1.4. Seja $k(\Gamma, \mathcal{A})$ uma ACG e seja $\gamma=a_{0} \beta_{1} a_{1} \ldots \beta_{r} a_{r}$ um $\mathcal{A}$-caminho em $k(\Gamma, \mathcal{A})$, onde cada $a_{i}$ é não-nulo. Definimos o caminho (ordinário) subjacente a $\gamma$ como sendo o caminho ordinário $\underline{\gamma}=\beta_{1} \ldots \beta_{n}$.

Por convenção, se algum $a_{i}$ for nulo, seu caminho subjacente é nulo.

Note que o comprimento de um $\mathcal{A}$-caminho é igual ao comprimento de seu caminho subjacente, caso este seja não-nulo.

Proposição 3.1.5. Suponha que $k(\Gamma, \mathcal{A})$ é uma ACG, e que $p_{1}, \ldots, p_{r}, q_{1}, \ldots, q_{s}$ são $\mathcal{A}$-caminhos em $k(\Gamma, \mathcal{A})$. Suponha que as somas $\sum_{i=1}^{r} p_{i}$ e $\sum_{i=1}^{s} q_{i}$ estejam na mesma classe de equivalência em $k(\Gamma, \mathcal{A})$. Então, para todo $i$ entre 1 e $r$, se $\underline{p_{i}} \neq 0$, existe $j$ entre 1 e $s$ tal que $\underline{p_{i}}=\underline{q_{j}}$.

Em particular, se $\gamma$ é um caminho sobre $\Gamma$, todo $\mathcal{A}$-caminho tendo $\gamma$ como caminho subjacente é não-nulo.

Demonstração. Se considerarmos as relações contidas na definição de ACG (Equações 3.1 e 3.2 acima), constatamos que elas envolvem apenas $\mathcal{A}$-caminhos com o mesmo caminho ordinário subjacente. Em virtude disso, não há como $\mathcal{A}$-caminhos com caminhos subjacentes distintos representarem a mesma classe de equivalência em $k(\Gamma, \mathcal{A})$. Disso segue a afirmação do enunciado.

Estabelecidas as definições acima, vamos enumerar algumas propriedades básicas das álgebras de caminhos generalizadas.

Observação 3.1.6. Mantenha as notações da Definição 3.1.1. É fácil se convencer de que uma álgebra de caminhos ordinária é um caso particular de ACG, pois se $A_{i}=k$ para todo $i \in \Gamma_{0}$, então $k(\Gamma, \mathcal{A}) \cong k \Gamma$.

Além disso, se $k(\Gamma, \mathcal{A})$ é uma ACG, então $k \Gamma$ é isomorfa a uma subálgebra de $k(\Gamma, \mathcal{A})$, basta identificar um caminho $\gamma_{1} \gamma_{2} \ldots \gamma_{t}$ sobre $\Gamma$ com o $\mathcal{A}$-caminho $1_{A_{s\left(\gamma_{1}\right)}} \gamma_{1} 1_{A_{e\left(\gamma_{1}\right)}} \gamma_{2} \ldots \gamma_{t} 1_{A_{e\left(\gamma_{t}\right)}}$. Essa subálgebra coincide com $k(\Gamma, \mathcal{A})$ se e somente se $A_{i}=k$ para todo $i \in Q_{0}$.

Note que $k(\Gamma, \mathcal{A})$ é uma álgebra associativa. E como sempre assumimos aqui que as aljavas são finitas, ela também tem um elemento identidade, que é dado por $\sum_{i \in \Gamma_{0}} 1_{A_{i}}$.

Observação 3.1.7. Para cada $i \in \Gamma_{0}$, seja $E_{i}$ um conjunto completo de idempotentes primitivos dois a dois ortogonais em $A_{i} \in \mathcal{A}$. Então é fácil ver que $\bigcup_{i \in \Gamma_{0}} E_{i}$ é um conjunto completo de idempotentes primitivos dois a dois ortogonais em $k(\Gamma, \mathcal{A})$. Usando o Teorema 2.2.19, vale a relação

$$
\operatorname{rk} K_{0}(k(\Gamma, \mathcal{A}))=\sum_{i \in \Gamma_{0}} \operatorname{rk} K_{0}\left(A_{i}\right)
$$


Proposição 3.1.8. (Compare com a Proposição 2.5.2). Com a notação da Definição $3.1 .1, k(\Gamma, \mathcal{A})$ tem dimensão finita sobre $k$ se e somente se todas as álgebras $A_{i}$ tiverem dimensão finita e se $\Gamma$ é uma aljava acíclica.

Demonstração. Se $\Gamma$ possui ciclos orientados, então um raciocínio bastante análogo ao que foi feito na demonstração da Proposição 2.5.2 mostra que $k(\Gamma, \mathcal{A})$ tem dimensão infinita. Da mesma forma, se alguma álgebra $A_{i}$ tem dimensão infinita para algum $i \in \Gamma_{0}$, então $k(\Gamma, \mathcal{A})$ tem dimensão infinita, já que um subconjunto infinito linearmente independente de $A_{i}$ pode ser visto como um conjunto de $\mathcal{A}$-caminhos de comprimento zero em $k(\Gamma, \mathcal{A})$.

Reciprocamente, se $\Gamma$ é acíclica, então, como é finita, há apenas um número finito de caminhos ordinários sobre $\Gamma$. Intercalando as flechas desses caminhos com elementos das bases finitas das álgebras $A_{i}$, obtemos um conjunto finito de $\mathcal{A}$-caminhos que gera $k(\Gamma, \mathcal{A})$. Isso mostra que $k(\Gamma, \mathcal{A})$ tem dimensão finita.

Observação 3.1.9. É um fato que uma ACG pode ser vista como uma álgebra tensorial, e como já observado antes, essa constatação será útil na formalização dos argumentos aqui. Este fato já aparece no artigo [7] onde as ACG's foram introduzidas. Mais tarde, isso foi novamente explorado em [10]. Vamos dar mais detalhes abaixo.

Como antes, seja $\Gamma$ uma aljava, e seja $\mathcal{A}=\left\{A_{i}: i \in \Gamma_{0}\right\}$ uma família de álgebras. Considere $A_{\mathcal{A}}=\oplus_{i \in \Gamma_{0}} A_{i}$, que é o produto direto das álgebras $A_{i}$.

Dada uma flecha $\alpha: i \rightarrow j \in \Gamma_{1}$, considere o espaço $M_{\alpha}$, que é o $\left(A_{i}-A_{j}\right)$-bimódulo gerado por $\{\alpha\}$. Então podemos tomar a soma direta de espaços $M_{\mathcal{A}}=\oplus_{\alpha \in \Gamma_{1}} M_{\alpha}$. Observe agora que $M_{\mathcal{A}}$ é na verdade um $\left(A_{\mathcal{A}}-A_{\mathcal{A}}\right)$-bimódulo: dados $\left(a_{i}\right)_{i \in \Gamma_{0}} \in A_{\mathcal{A}}$ e $\left(m_{\alpha}\right)_{\alpha \in \Gamma_{1}} \in M_{\mathcal{A}}$, as ações à esquerda e à direita são dadas por:

$$
\begin{aligned}
& \left(a_{i}\right)_{i} \cdot\left(m_{\alpha}\right)_{\alpha}=\left(a_{s(\alpha)} m_{\alpha}\right)_{\alpha} \\
& \left(m_{\alpha}\right)_{\alpha} \cdot\left(a_{i}\right)_{i}=\left(m_{\alpha} a_{e(\alpha)}\right)_{\alpha}
\end{aligned}
$$

Então podemos considerar a álgebra tensorial

$$
\begin{aligned}
k(\Gamma, \mathcal{A}) & =T\left(A_{\mathcal{A}}, M_{\mathcal{A}}\right) \\
& \doteq A_{\mathcal{A}} \oplus M_{\mathcal{A}} \oplus\left(M_{\mathcal{A}} \otimes_{A_{\mathcal{A}}} M_{\mathcal{A}}\right) \oplus\left(M_{\mathcal{A}} \otimes_{A_{\mathcal{A}}} M_{\mathcal{A}} \otimes_{A_{\mathcal{A}}} M_{\mathcal{A}}\right) \oplus \ldots
\end{aligned}
$$

Que, como já visto antes, é um $\left(A_{\mathcal{A}}-A_{\mathcal{A}}\right)$-bimódulo, tem $A_{\mathcal{A}}$ como subálgebra, e portanto seu elemento identidade coincide com o de $A_{\mathcal{A}}$. Além disso, esta álgebra tensorial é isomorfa à ACG da Definição 3.1.1.

\subsection{O radical de uma ACG}

Também no artigo [7], os autores descrevem como é o radical de uma álgebra de caminhos generalizada. Dedicamos esta seção para discutir este resultado. 
Definição 3.2.1. Seja $\Gamma$ uma aljava, e seja $\mathcal{A}=\left\{A_{i}: i \in \Gamma_{0}\right\}$ uma família de álgebras.

- Um $\mathcal{A}$-caminho regular de comprimento zero é um elemento de $\operatorname{rad} A_{i}$, para algum vértice $i \in \Gamma_{0}$ pelo qual não passe nenhum ciclo orientado de $\Gamma$.

- Para $n>0$ natural, um $\mathcal{A}$-caminho regular de comprimento $n$ é um $\mathcal{A}$-caminho $\gamma$ de comprimento $n$ em $k(\Gamma, \mathcal{A})$ tal que o caminho ordinário subjacente $\underline{\gamma}$ não seja subcaminho de nenhum ciclo orientado de $\Gamma$.

Teorema 3.2.2. ([7]) Sejam $\Gamma$ uma aljava, $\mathcal{A}=\left\{A_{i}: i \in \Gamma_{0}\right\}$ uma família de álgebras e $\Lambda=k(\Gamma, \mathcal{A})$. Seja $M$ o subespaço vetorial de $\Lambda$ gerado pelos $\mathcal{A}$-caminhos regulares. Então $M$ coincide com o radical de $\Lambda$.

Lema 3.2.3. Seja $A$ uma $k$-álgebra e sejam $J, I$ dois ideais bilaterais de $A$ tais que $J \subseteq I \subseteq A$. Se $J$ é quasirregular em $A$ e $I / J$ é quasirregular em $A / J$, então $I$ é quasirregular em $A$.

Demonstração. Seja $a \in I$. Temos de provar que $1-a$ é invertível em $A$. Temos que $\bar{a} \in I / J$. Pela hipótese, $\overline{1}-\bar{a}$ é invertível em $A / J$, logo existe $\bar{b} \in A / J$ tal que $\bar{b}(\overline{1}-\bar{a})=(\overline{1}-\bar{a}) \bar{b}=\overline{1}$. Daí $\overline{1-b+b a}=\overline{1-b+a b}=\overline{0}$, ou seja, $1-b+b a, 1-b+a b \in J$. Pela hipótese novamente, $1-b+b a=1-(b-b a)$ e $1-b+a b=1-(b-a b)$ são quasirregulares, e portanto $b-b a$ e $b-a b$ são invertíveis em $A$. Logo existem $c, d \in A$ tais que $c(b-b a)=(b-a b) d=1 \Rightarrow c b-c b a=b d-a b d=$ $1 \Rightarrow c b(1-a)=(1-a) b d=1$. Portanto $1-a$ tem inverso à esquerda e à direita, logo estes inversos coincidem e $1-a$ é invertível.

Demonstração do Teorema 3.2.2. Queremos provar que $\operatorname{rad} \Lambda=M$.

Dado $X \subseteq \Lambda$ um subconjunto, usamos a notação $(X)$ para denotar o ideal bilateral de $\Lambda$ gerado por $X$.

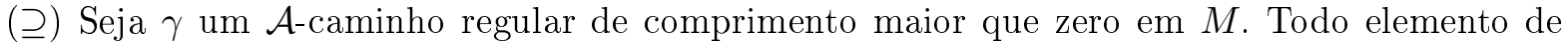
$(\gamma)^{2}$ é soma de elementos da forma $a \gamma b a^{\prime} \gamma b^{\prime}$, onde $a, b, a^{\prime}, b^{\prime}$ são todos $\mathcal{A}$-caminhos. Se $a \gamma b a^{\prime} \gamma b^{\prime} \neq 0$, então em particular $\gamma b a^{\prime} \neq 0$ e $\underline{\gamma} \underline{b a^{\prime}} \neq 0$, o que é absurdo, porque $\underline{\gamma} \underline{b a^{\prime}}$ é um ciclo orientado e $\gamma$ é regular. Portanto $(\gamma)^{2}=0$, o que significa que $(\gamma)$ é um ideal nilpotente e portanto quasirregular pela Observação 2.2.10. Pela Proposição 2.2.11, segue que $(\gamma) \subseteq \operatorname{rad} \Lambda$.

Seja agora $i$ um vértice pelo qual não passe ciclo orientado. Seja $U_{i}$ o ideal de $\Lambda$ gerado pe$\operatorname{los} \mathcal{A}$-caminhos que começam ou terminam em $i$. Então $\left(\operatorname{rad}\left(A_{i}\right)\right)=\operatorname{rad} A_{i}+U_{i}$. Note que $U_{i}$ é um ideal quasirregular, a demonstração desse fato sendo análoga à demonstração para $(\gamma)$ feita acima. Temos ainda que $\frac{\operatorname{rad} A_{i}+U_{i}}{U_{i}} \cong \operatorname{rad} A_{i}$ é quasirregular. Portanto, pelo Lema 3.2.3, segue que $\left(\operatorname{rad} A_{i}\right)$ é quasirregular e que portanto, pela Proposição 2.2.11, $\left(\operatorname{rad} A_{i}\right) \subseteq \operatorname{rad} \Lambda$. Isso completa a demonstração de que $M \subseteq \operatorname{rad} \Lambda$.

$(\subseteq)$ Suponha por absurdo que $M \varsubsetneqq \operatorname{rad} \Lambda$. Então existe $x \in \operatorname{rad} \Lambda \backslash M$. Vamos escrever $x=$ $\sum_{l=1}^{n} p_{l}$, onde cada $p_{l}$ é um $\mathcal{A}$-caminho, com $p_{l}$ não-regular para $1 \leq l \leq m$ e com $p_{l}$ regular para $m<l \leq n$. Mas daí $x-\sum_{l=m+1}^{n} p_{l}=\sum_{l=1}^{m} p_{l}$ pertence a rad $\Lambda$ e não a $M$. Portanto, trocando $x$ se necessário, podemos supor sem perda de generalidade que $p_{l}$ é não-regular para todo $l$.

Como $x \neq 0$ (pois senão $x \in M$ ), $1_{\Lambda} x 1_{\Lambda} \neq 0 \Rightarrow \sum_{r, s \in \Gamma_{0}} 1_{A_{r}} x 1_{A_{s}} \neq 0$ e logo existem $r, s \in \Gamma_{0}$ tais que $1_{A_{r}} x 1_{A_{s}} \neq 0$. Como $\operatorname{rad} \Lambda$ é um ideal bilateral, $1_{A_{r}} x 1_{A_{s}} \in \operatorname{rad} \Lambda$, e portanto $1_{A_{r}} x 1_{A_{s}}=$ $\sum_{l=1}^{n} 1_{A_{r}} p_{l} 1_{A_{s}}$ é não-nulo e pertence a $\operatorname{rad} \Lambda$. Ou seja, podemos supor sem perda de generalidade que cada $p_{l}$ é um $\mathcal{A}$-caminho que começa em $r$ e termina em $s$. 
Como $p_{l}$ não é regular, $\underline{p_{l}}$ é subcaminho de um ciclo orientado, logo existe um caminho $q: s \rightsquigarrow r$ sobre $\Gamma$. Daí $x q \in \operatorname{rad} \Lambda$ e $x q \neq 0$ e $\operatorname{logo} \sum_{l=1}^{n} p_{l} q \neq 0$ é um elemento de $\operatorname{rad} \Lambda$. Isso mostra que podemos supor sem perda de generalidade que $p_{l}: r \rightsquigarrow r$ é um ciclo orientado para todo $l$.

Seja $\gamma=\alpha \ldots \beta: r \rightsquigarrow r$ um ciclo orientado fixo sobre o vértice $r$ (já vimos que existe pelo menos um). Então $\gamma x \gamma \neq 0$ pertence a $\operatorname{rad} \Lambda$, e portanto $\sum_{l=1}^{n} \gamma p_{l} \gamma \neq 0$ e $\sum_{l=1}^{n} \gamma p_{l} \gamma \in \operatorname{rad} \Lambda$. Ou seja, finalmente, podemos supor sem perda de generalidade que cada $p_{l}$ é da forma $\gamma f_{l} \gamma$, onde cada $f_{l}: r \rightsquigarrow r$ é um ciclo orientado.

Como $x \in \operatorname{rad} \Lambda,-x \in \operatorname{rad} \Lambda$ e pela Proposição 2.2.11, $-x$ é quasirregular. Logo existe $z \in \Lambda$ tal que $(1-(-x)) z=1 \Rightarrow(1+x) z=1$. Seja $y=z-1$. Então $z=1+y \Rightarrow(1+x)(1+y)=1 \Rightarrow$ $1+x+y+x y=1 \Rightarrow x+y+x y=0$. Multiplicando dos dois lados por $1_{A_{r}}$ e lembrando que $x$ é soma de $\mathcal{A}$-caminhos que começam e terminam em $r$, temos que $1_{A_{r}}(x+y+x y) 1_{A_{r}}=1_{A_{r}} \cdot 0.1_{A_{r}} \Rightarrow 1_{A_{r}} x 1_{A_{r}}+$ $1_{A_{r}} y 1_{A_{r}}+\left(1_{A_{r}} x\right) y 1_{A_{r}}=0 \Rightarrow x+1_{A_{r}} y 1_{A_{r}}+\left(x 1_{A_{r}}\right) y 1_{A_{r}}=0 \Rightarrow x+1_{A_{r}} y 1_{A_{r}}+x\left(1_{A_{r}} y 1_{A_{r}}\right)=0$. Seja então $y^{\prime}=1_{A_{r}} y 1_{A_{r}}$. Daí $y^{\prime}=1_{A_{r}} y^{\prime} 1_{A_{r}}$ e $x+y^{\prime}+x y^{\prime}=0$. Podemos escrever $y^{\prime}=\sum_{j=1}^{t} q_{j}$, onde cada $q_{j}$ é um $\mathcal{A}$-caminho não-nulo $r \rightsquigarrow r$.

Por um lado, $x+y^{\prime}=\sum_{l=1}^{n} p_{l}+\sum_{j=1}^{t} q_{j}$, e por outro, $x y^{\prime}=\sum_{l=1}^{n} \sum_{j=1}^{t} p_{l} q_{j}$. Como $x+y^{\prime}=-x y^{\prime}$, segue que

$$
\sum_{l=1}^{n} p_{l}+\sum_{j=1}^{t} q_{j}=-\sum_{l=1}^{n} \sum_{j=1}^{t} p_{l} q_{j}
$$

Vejamos porque esta última igualdade resulta em uma contradição. Como $p_{l}=\gamma f_{l} \gamma$ para cada $l$, cada $p_{l}$ tem comprimento menor ou igual a 2. Logo cada $p_{l} q_{j}$ tem comprimento menor ou igual a 2, já que $q_{j} \neq 0$ por construção. Se todos os $q_{j}$ 's tiverem comprimento 0 ou 1, obtemos uma contradição pela Proposição 3.1.5, já que não poderá existir $l$ e $j$ tais que $\underline{q_{1}}=\underline{p_{l} q_{j}}$, sendo o comprimento de $p_{l} q_{j}$ sempre estritamente maior do que o de $\underline{q_{1}}$. Logo pelo menos algum $q_{j}$ tem comprimento maior ou igual a 2. Também pela Proposição 3.1.5, temos que:

$$
\max _{1 \leq l \leq n, 1 \leq j \leq t}\left\{l\left(p_{l}\right), l\left(q_{l}\right)\right\}=\max _{1 \leq l \leq n}\left\{l\left(p_{l}\right)\right\}+\max _{1 \leq j \leq t}\left\{l\left(q_{l}\right)\right\}
$$

E como cada máximo do lado direito vale pelo menos 2, esta última igualdade leva facilmente a um absurdo.

Exemplo 3.2.4. Vamos retomar o Exemplo 3.1.3. Naquele exemplo a aljava $\Gamma$ era

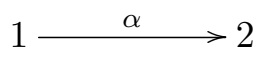

e a família de álgebras era $\mathcal{A}=\left\{T_{2}(k), k\right\}$. Vamos calcular o radical de $k(\Gamma, \mathcal{A})$ usando o Teorema 3.2.2 acima. Note que $\Gamma$ é acíclica, de modo que nenhum caminho dessa aljava é subcaminho de um ciclo orientado.

Como $\operatorname{rad} T_{2}(k)$ é o subespaço gerado por $\left(\begin{array}{ll}0 & 1 \\ 0 & 0\end{array}\right)$ e $\operatorname{rad} k=0$, os $\mathcal{A}$-caminhos regulares de comprimento zero estão todos no subespaço gerado por $\left(\begin{array}{ll}0 & 1 \\ 0 & 0\end{array}\right)$.

O único caminho de comprimento maior que zero sobre $\Gamma$ é $\alpha$, de modo que os únicos $\mathcal{A}$-caminhos regulares de comprimento maior que zero são os $\mathcal{A}$-caminhos que incluem $\alpha$, e estes por sua vez 
são combinações lineares dos elementos $\left(\begin{array}{ll}1 & 0 \\ 0 & 0\end{array}\right) \alpha,\left(\begin{array}{ll}0 & 1 \\ 0 & 0\end{array}\right) \alpha$ e $\left(\begin{array}{ll}0 & 0 \\ 0 & 1\end{array}\right) \alpha$. Portanto o radical de $k(\Gamma, \mathcal{A})$ é o subespaço gerado pelo conjunto abaixo:

$$
\left\{\left(\begin{array}{ll}
0 & 1 \\
0 & 0
\end{array}\right),\left(\begin{array}{ll}
1 & 0 \\
0 & 0
\end{array}\right) \alpha,\left(\begin{array}{ll}
0 & 1 \\
0 & 0
\end{array}\right) \alpha,\left(\begin{array}{ll}
0 & 0 \\
0 & 1
\end{array}\right) \alpha\right\}
$$




\section{Capítulo 4}

\section{Generalização: ACG's com relações}

\section{1 Álgebras de Caminhos Generalizadas com Relações}

Com o intuito de obter mais resultados posteriormente, vamos estender o conceito de álgebras de caminhos generalizadas (ACG), de modo a permitir que elas tenham relações. Ao fazer isso, essas álgebras serão chamadas de álgebras de caminhos generalizadas com relações.

A ideia de considerar o quociente de uma ACG por um ideal gerado por relações já foi estudada por Li Fang (veja [10] por exemplo, ou o Apêndice A.3). No entanto, o conceito que vamos introduzir aqui é um pouco diferente. Nossa preferência pelo conceito aqui apresentado vem da possibilidade de, a partir dele, generalizar o Teorema 4.2.3 abaixo.

Definição 4.1.1. Seja $k(\Gamma, \mathcal{A})$ uma $\mathrm{ACG}$, onde $\Gamma$ é um aljava e $\mathcal{A}=\left\{k \Sigma_{i} / \Omega_{i}: i \in \Gamma_{0}\right\}$ é uma família de álgebras de caminhos com relações (aqui $\Sigma_{i}$ denota uma aljava e $\Omega_{i}$ denota um ideal admissível de $k \Sigma_{i}$ ).

Seja $I$ um conjunto finito de relações sobre $\Gamma$ que gera um ideal admissível. Então consideramos o seguinte subconjunto de $k(\Gamma, \mathcal{A})$ :

$$
\begin{aligned}
\mathcal{A}(I) & =\left\{\sum_{i=1}^{t} \lambda_{i} \beta_{i 1} \overline{\gamma_{i 1}} \beta_{i 2} \ldots \overline{\gamma_{i\left(m_{i}-1\right)}} \beta_{i m_{i}}:\right. \\
& \left.\sum_{i=1}^{t} \lambda_{i} \beta_{i 1} \ldots \beta_{i m_{i}} \text { é uma relação em } I \text { e } \gamma_{i j} \text { é um caminho em } \Sigma_{e\left(\beta_{i j}\right)}\right\}
\end{aligned}
$$

Com isso, o quociente $\frac{k(\Gamma, \mathcal{A})}{(\mathcal{A}(I))}$ é chamado de álgebra de caminhos generalizada com relações (ACGR). Para simplificar a notação, escrevemos $\frac{k(\Gamma, \mathcal{A})}{(\mathcal{A}(I))}=k(\Gamma, \mathcal{A}, I)$. Além disso, quando estiver claro a partir do contexto, podemos denotar o conjunto $\mathcal{A}(I)$ simplesmente por $I$.

Exemplo 4.1.2. Seja $\Lambda=k(\Gamma, \mathcal{A}, I)$ uma ACGR, onde $\Gamma$ é a aljava

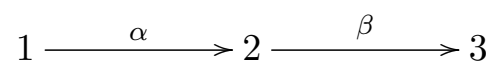

e onde $I=\{\alpha \beta\}$ e $\mathcal{A}=\left\{A_{1}, A_{2}, A_{3}\right\}$, com $A_{1}=A_{3}=k$ e $A_{2}=k \Sigma_{2} / \Omega_{2}$, onde $\Sigma_{2}$ é a aljava 


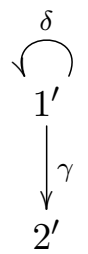

e $\Omega_{2}=\left(\delta^{2}\right)$. Note que os caminhos $\epsilon_{1^{\prime}}, \epsilon_{2^{\prime}}, \gamma, \delta$ e $\delta \gamma$, onde $\epsilon_{i}$ denota o caminho de comprimento zero no vértice $i$, são os únicos que não são nulos em $A_{2}$.

Então neste caso o conjunto $\mathcal{A}(I)$ é igual a $\left\{\alpha \overline{\epsilon_{1^{\prime}}} \beta, \alpha \overline{\epsilon_{2^{\prime}}} \beta, \alpha \bar{\gamma} \beta, \alpha \bar{\delta} \beta, \alpha \overline{\delta \gamma} \beta, \alpha \overline{\delta^{2}} \beta=0\right\}$, de modo que os elementos desse conjunto serão iguais a zero em $k(\Gamma, \mathcal{A}, I)$. Note que não precisamos incluir a relação $\delta^{2}=0 \mathrm{em} \mathcal{A}(I)$, porque o elemento $\delta^{2}$ já é zero dentro de $A_{2}$ : por definição, $\mathcal{A}(I)$ contém apenas relações obtidas a partir das relações sobre $\Gamma$.

\subsection{Realizando uma ACGR como uma álgebra de caminhos}

Já que uma ACGR é uma álgebra, faz sentido aplicar o Teorema de Gabriel (2.6.1) a ela, e assim obter uma aljava ordinária com um conjunto de relações. Isto vai ser exatamente o conteúdo do Teorema 4.2.5 abaixo, que é o resultado principal desta seção.

O Teorema 4.2.5 será uma generalização de um resultado de 2008 obtido por Ibáñez-Cobos, Navarro and López-Peña ([6]), cujos enunciado e demonstração serão apresentados a seguir. A diferença entre o teorema presente em [6] e o Teorema 4.2.5 é que o primeiro descreve a aljava ordinária de uma ACG e o outro, como já colocado, faz o mesmo para uma ACGR.

Seja $\Lambda=k(\Gamma, \mathcal{A})$ uma álgebra de caminhos generalizada, com $\mathcal{A}=\left\{A_{i}: i \in \Gamma_{0}\right\}$. Então, pelo Teorema de Gabriel (Teorema 2.6.1), existe, para cada $i$, uma aljava $\Sigma_{i}$ e um ideal admissível $\Omega_{i}$ de $k \Sigma_{i}$ tal que $A_{i} \cong k \Sigma_{i} / \Omega_{i}$.

Vamos agora construir uma aljava $Q^{\prime}$ e provar que ela coincide com a aljava de Gabriel de $\Lambda$, denotada por $Q_{\Lambda}$. A aljava $Q^{\prime}$ é definida abaixo:

- O conjunto de vértices de $Q^{\prime}$ é $\bigcup_{i \in \Gamma_{0}}\left(\Sigma_{i}\right)_{0}$.

- Se $a$ é um vértice de $\Sigma_{i}$ e $b$ é um vértice de $\Sigma_{j}$ então o número de flechas da forma $a \rightarrow b$ em $Q^{\prime}$ é igual ao número de flechas da forma $a \rightarrow b$ em $\Sigma_{i}$ se $i=j$, e é igual ao número de flechas da forma $i \rightarrow j$ em $\Gamma$ se $i \neq j$. Simbolicamente:

$$
[a, b]_{Q^{\prime}}= \begin{cases}{[a, b]_{\Sigma_{i}}} & , \text { se } i=j \\ {[i, j]_{\Gamma}} & , \text { se } i \neq j\end{cases}
$$

Exemplo 4.2.1. Vamos retomar mais uma vez o Exemplo 3.1.3. Usando as notações acima:

$$
\begin{gathered}
\Gamma=1 \longrightarrow 2 \quad \Sigma_{1}=\bullet_{e_{1}} \longrightarrow \bullet_{e_{2}} \quad \Sigma_{2}=\bullet_{f} \\
A_{1}=k \Sigma_{1} \cong T_{2}(k) \quad A_{2}=k \Sigma_{2} \cong k
\end{gathered}
$$

Vamos obter a aljava $Q^{\prime}$ tal como definida acima. Em primeiro lugar, $\left(Q^{\prime}\right)_{0}=\left(\Sigma_{1}\right)_{0} \cup\left(\Sigma_{2}\right)_{0}=$ $\left\{e_{1}, e_{2}, f\right\}$. 
Calculando as flechas entre vértices que vieram da mesma aljava:

$$
\begin{aligned}
{\left[e_{1}, e_{1}\right]_{Q^{\prime}}=\left[e_{1}, e_{1}\right]_{\Sigma_{1}}=0 } & {\left[e_{2}, e_{2}\right]_{Q^{\prime}}=\left[e_{2}, e_{2}\right]_{\Sigma_{1}}=0 } \\
{\left[e_{1}, e_{2}\right]_{Q^{\prime}}=\left[e_{1}, e_{2}\right]_{\Sigma_{1}}=1 } & {\left[e_{2}, e_{1}\right]_{Q^{\prime}}=\left[e_{2}, e_{1}\right]_{\Sigma_{1}}=0 } \\
{[f, f]_{Q^{\prime}}=[f, f]_{\Sigma_{2}}=0 } &
\end{aligned}
$$

Calculando as flechas entre vértices que vêm de aljavas diferentes:

$$
\begin{array}{ll}
{\left[e_{1}, f\right]_{Q^{\prime}}=[1,2]_{\Gamma}=1} & {\left[e_{2}, f\right]_{Q^{\prime}}=[1,2]_{\Gamma}=1} \\
{\left[f, e_{1}\right]_{Q^{\prime}}=[2,1]_{\Gamma}=0} & {\left[f, e_{2}\right]_{Q^{\prime}}=[2,1]_{\Gamma}=0}
\end{array}
$$

Juntando todas as informações, concluímos que a aljava $Q^{\prime}$ é igual a:

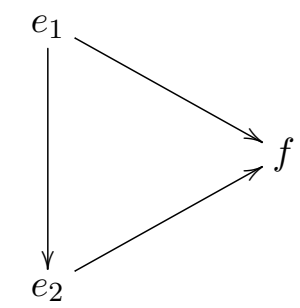

Exemplo 4.2.2. Considere a $\mathrm{ACG} k(\Gamma, \mathcal{A})$ onde $\Gamma$ é dada por:

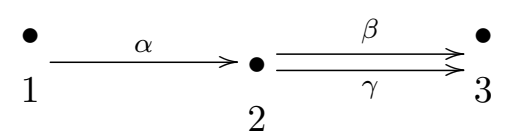

e onde também $\mathcal{A}=\left\{A_{1}=k \Sigma_{1} / \Omega_{1}, A_{2}=k \Sigma_{2} / \Omega_{2}, A_{3}=k \Sigma_{3} / \Omega_{3}\right\}$, sendo $\Sigma_{1}=\Sigma_{3}=\bullet$, $\Omega_{1}=\Omega_{3}=0$ (e portanto $A_{1}=A_{3}=k$ ), sendo $\Sigma_{2}$ a aljava

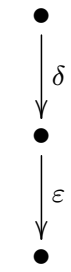

e sendo $\Omega_{2}=(\delta \varepsilon)$. Para calcular a aljava $Q^{\prime}$, podemos proceder como no Exemplo 4.2.1 acima, mas na prática fazemos um raciocínio mais breve: note que cada $\Sigma_{i}$ é uma subaljava plena de $Q^{\prime}$, e que para cada flecha $i \rightarrow j$ em $\Gamma$ corresponde uma coleção de flechas, uma ligando cada vértice de $\Sigma_{i}$ a cada vértice de $\Sigma_{j}$. Neste caso a flecha $\alpha$ dá origem a três flechas, assim como $\beta$ e $\gamma$. Com isto podemos concluir que a aljava $Q^{\prime}$ é dada por: 


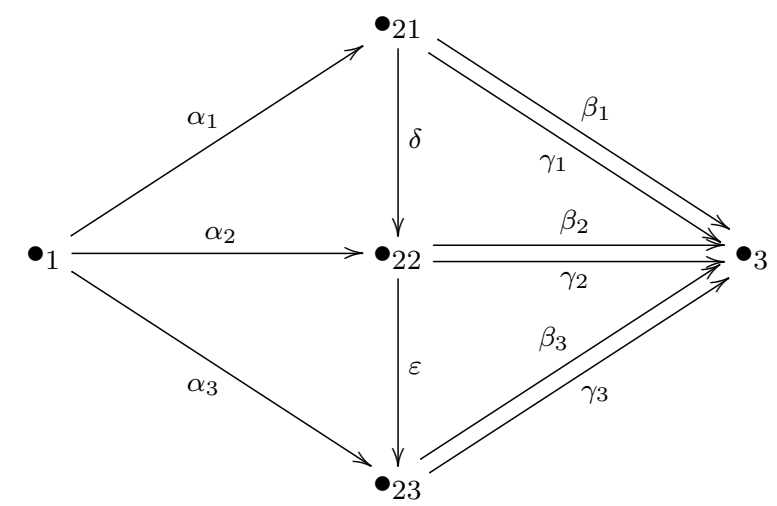

Teorema 4.2.3. ([6],3.3) Sendo $Q^{\prime}$ a aljava definida acima, se $\Gamma$ é acíclica então $Q^{\prime}$ coincide com a aljava de Gabriel $Q_{\Lambda}$ de $\Lambda$ e existe um isomorifsmo

$$
\Lambda \doteq k(\Gamma, \mathcal{A}) \cong k Q_{\Lambda} / \Omega_{\Lambda}
$$

onde $\Omega_{\Lambda}$ é o ideal gerado por $\cup_{i \in \Gamma_{0}} \Omega_{i}$.

Demonstração. Antes de tudo, precisamos verificar que o Teorema de Gabriel (2.6.1) se aplica à algebra $k(\Gamma, \mathcal{A})$. Como pelo enunciado $\Gamma$ é acíclica e estamos sempre supondo que as álgebras $A_{i} \in A$ têm dimensão finita, segue pela Proposição 3.1.8 que $k(\Gamma, \mathcal{A})$ tem dimensão finita. Além disso, também já estamos supondo que $k$ é um corpo algebricamente fechado. Portanto, para podermos aplicar o Teorema de Gabriel, resta ver que $k(\Gamma, \mathcal{A})$ é básica.

Vamos usar a caracterização da Proposição 2.2.14. Como consequência do Teorema 3.2.2, o quociente $\Lambda / \operatorname{rad} \Lambda$ é isomorfo ao produto direto $\oplus_{i \in \Gamma_{0}} A_{i} / \operatorname{rad} A_{i}$. Para cada $i \in \Gamma_{0}$, estamos supondo que $A_{i}$ é básica, portanto $A_{i} / \operatorname{rad} A_{i}$ é isomorfo ao produto direto de cópias de $k$, e portanto a própria álgebra quociente $\Lambda / \operatorname{rad} \Lambda$ é isomorfa a um produto direto de cópias de $k$. Portanto $\Lambda$ é básica.

Sendo assim, pelo Teorema de Gabriel, $\Lambda$ tem uma aljava de Gabriel, que já escolhemos denotar por $Q_{\Lambda}$. O próximo passo é mostrar que $Q^{\prime}$ é igual a $Q_{\Lambda}$.

Para cada $i \in \Gamma_{0}$, seja $E_{i}=\left\{e_{i}^{1}, \ldots, e_{i}^{c_{i}}\right\}$ um conjunto completo de idempotentes primitivos doisa-dois ortogonais em $A_{i}$. Lembramos que, pela demonstração do Teorema 2.6.1, podemos assumir que $\left(\Sigma_{i}\right)_{0}=E_{i}$.

Sabemos que $E \doteq \cup_{i \in \Gamma_{0}} E_{i}=\cup_{i \in \Gamma_{0}}\left(\Sigma_{i}\right)_{0}$ é um conjunto completo de idempotentes primitivos dois-a-dois ortogonais em $\Lambda$. Logo, pela maneira que as duas aljavas são construídas, podemos concluir que $\left(Q_{\Lambda}\right)_{0}=E=\left(Q^{\prime}\right)_{0}$.

Vamos ver que $Q_{1}=\left(Q_{\Lambda}\right)_{1}$. Para tanto, vamos descrever os elementos de $\operatorname{rad} \Lambda /(\operatorname{rad} \Lambda)^{2}$. Nas considerações abaixo, lembramos que $\Gamma$ é acíclica e temos em mente o Teorema 3.2.2.

- Um $\mathcal{A}$-caminho de comprimento 0 em $(\operatorname{rad} \Lambda)^{2}$ só pode ser um elemento de $\left(\operatorname{rad} A_{i}\right)^{2}$, para algum $i \in \Gamma_{0}$. Portanto, as classes de equivalência dos $\mathcal{A}$-caminhos de comprimento zero em $\operatorname{rad} \Lambda /(\operatorname{rad} \Lambda)^{2}$ correspondem aos elementos de $\cup_{i \in \Gamma_{0}} \operatorname{rad} A_{i} /\left(\operatorname{rad} A_{i}\right)^{2}$.

- Um $\mathcal{A}$-caminho de comprimento 1 em $(\operatorname{rad} \Lambda)^{2}$ é um $\mathcal{A}$-caminho da forma $a \alpha b$, onde $\alpha: i \rightarrow j$ é uma flecha em $\Gamma$ e ou $a \in \operatorname{rad} A_{i}$ ou $b \in \operatorname{rad} A_{j}$. Portanto, as classes de equivalência dos $\mathcal{A}$-caminhos de comprimento $1 \mathrm{em} \operatorname{rad} \Lambda /(\operatorname{rad} \Lambda)^{2}$ têm forma $a \alpha b$, onde $\alpha: i \rightarrow j$ é uma flecha em $\Gamma, a \in A_{i} / \operatorname{rad} A_{i}$ e $b \in A_{j} / \operatorname{rad} A_{j}$. Este espaço será denotado por $\mathcal{J}_{1}$. 
- Todo $\mathcal{A}$-caminho de comprimento maior do que 1 pertence $(\operatorname{rad} \Lambda)^{2}$, portanto sua classe de equivalência é nula em $\operatorname{rad} \Lambda /(\operatorname{rad} \Lambda)^{2}$.

Em suma, o quociente $\operatorname{rad} \Lambda /(\operatorname{rad} \Lambda)^{2}$ é gerado pelo conjunto $\cup_{i \in \Gamma_{0}} \operatorname{rad} A_{i} /\left(\operatorname{rad} A_{i}\right)^{2}$ unido ao conjunto das classes de $\mathcal{A}$-caminhos da forma $a \alpha b$, onde $\alpha: i \rightarrow j$ é uma flecha em $\Gamma, a \in A_{i} / \operatorname{rad} A_{i}$ e $b \in A_{j} / \operatorname{rad} A_{j}$.

Sejam $e_{i}^{j}, e_{l}^{m} \in E$ dois vérices de $Q_{\Lambda}$. Para estudar o número de flechas entre os dois, dividimos em dois casos:

- Se $i=l$, então $e_{i}^{j}$ e $e_{i}^{m}$ pertencem ambos a $\left(\Sigma_{i}\right)_{0}$. Daí, usando a demonstração do Teorema de Gabriel:

$$
\left[e_{i}^{j}, e_{i}^{m}\right]_{Q_{\Lambda}}=\operatorname{dim}_{k} e_{i}^{j}\left(\frac{\operatorname{rad} \Lambda}{\operatorname{rad} \Lambda^{2}}\right) e_{i}^{m}=\operatorname{dim}_{k} e_{i}^{j}\left(\frac{\operatorname{rad} A_{i}}{\operatorname{rad} A_{i}^{2}}\right) e_{i}^{m}=\left[e_{i}^{j}, e_{i}^{m}\right]_{\Sigma_{i}}
$$

Ou seja, o número de flechas entre $e_{i}^{j}$ e $e_{i}^{m}$ é o mesmo em $Q_{\Lambda}$ e em $\Sigma_{i}$.

- Se $i \neq l$, então, novamente usando a demonstração do Teorema de Gabriel:

$$
\begin{aligned}
{\left[e_{i}^{j}, e_{i}^{m}\right]_{Q_{\Lambda}} } & =\operatorname{dim}_{k} e_{i}^{j}\left(\frac{\operatorname{rad} \Lambda}{\operatorname{rad} \Lambda^{2}}\right) e_{l}^{m} \\
& =\operatorname{dim}_{k} e_{i}^{j}\left(\mathcal{J}_{1}\right) e_{l}^{m} \\
& =\sum_{\alpha: i \rightarrow l \in \Gamma_{1}} \operatorname{dim}_{k} e_{i}^{j}\left(\frac{A_{i}}{\operatorname{rad} A_{i}}\right) \alpha\left(\frac{A_{l}}{\operatorname{rad} A_{l}}\right) e_{l}^{m} \\
& =\sum_{\alpha: i \rightarrow l \in \Gamma_{1}} \operatorname{dim}_{k} e_{i}^{j}\left(\frac{A_{i}}{\operatorname{rad} A_{i}}\right) \cdot \operatorname{dim}_{k}\left(\frac{A_{l}}{\operatorname{rad} A_{l}}\right) e_{l}^{m} \\
& =\sum_{\alpha: i \rightarrow l \in \Gamma_{1}} \sum_{s=1}^{c_{i}} \sum_{t=1}^{c_{l}} \operatorname{dim}_{k} e_{i}^{j}\left(\frac{A_{i}}{\operatorname{rad} A_{i}}\right) e_{i}^{s} \cdot \operatorname{dim}_{k} e_{l}^{t}\left(\frac{A_{l}}{\operatorname{rad} A_{l}}\right) e_{l}^{m} \\
& =\sum_{\alpha: i \rightarrow l \in \Gamma_{1}} \sum_{s=1}^{c_{i}} \sum_{t=1}^{c_{l}} \delta_{j, s} \delta_{m, t} \\
& =\sum_{\alpha: i \rightarrow l \in \Gamma_{1}} 1=[i, l]_{\Gamma}
\end{aligned}
$$

Isso conclui a demonstração de que $Q^{\prime}=Q_{\Lambda}$. Resta provar que o quociente da álgebra de caminhos $k Q_{\Lambda}$ pelo ideal $\Omega_{\Lambda}$ do enunciado é isomorfo a $\Lambda$.

Para cada $i \in \Gamma_{0}, k \Sigma_{i} / \Omega_{i} \cong A_{i}$. Portanto existe, para cada $i$, um epimorfismo de álgebras $f_{i}: k \Sigma_{i} \rightarrow A_{i}$ tal que Ker $f_{i}=\Omega_{i}$.

O subespaço de $k Q_{\Lambda}$ gerado por $E$, denotado por $\left(k Q_{\Lambda}\right)_{0}$, é uma subálgebra de $k Q_{\Lambda}$. Além disso, podemos definir um homomorfismo de álgebras $g_{0}:\left(k Q_{\Lambda}\right)_{0} \rightarrow \Lambda$ colocando $g_{0}\left(e_{i}^{j}\right)=f_{i}\left(e_{i}^{j}\right)=e_{i}^{j}$ para todo $e_{i}^{j} \in E$. Por restrição de escalares via $g_{0}, \Lambda$ é um $\left(\left(k Q_{\Lambda}\right)_{0}-\left(k Q_{\Lambda}\right)_{0}\right)$-bimódulo.

Seja $\left(k Q_{\Lambda}\right)_{1}$ o subespaço de $k Q_{\Lambda}$ gerado pelas flechas de $Q_{\Lambda}$. Este é naturalmente um $\left(\left(k Q_{\Lambda}\right)_{0}-\right.$ $\left.\left(k Q_{\Lambda}\right)_{0}\right)$-bimódulo. Podemos definir uma aplicação $g_{1}:\left(k Q_{\Lambda}\right)_{1} \rightarrow \Lambda$ estabelecendo que, para cada flecha $\alpha: e_{i}^{j} \rightarrow e_{l}^{m}$ em $Q_{\Lambda}$, 


$$
g_{1}(\alpha)= \begin{cases}f_{i}(\alpha) & , \text { se } i=l \\ e_{l}^{m} \alpha e_{i}^{j} & , \text { se } i \neq l\end{cases}
$$

Note ainda que $g_{1}$ é um morfismo de $\left(\left(k Q_{\Lambda}\right)_{0}-\left(k Q_{\Lambda}\right)_{0}\right)$-bimódulos. Dessa forma, usando o fato de que $k Q_{\Lambda} \cong T\left(\left(k Q_{\Lambda}\right)_{0},\left(k Q_{\Lambda}\right)_{1}\right)$ (vide Observação 3.1.9) e a Propriedade Universal das Álgebras Tensoriais (Proposição 2.2.4) com as aplicações $g_{0}$ e $g_{1}$, existe um homomorfismo de álgebras $g: k Q_{\Lambda} \rightarrow k(\Gamma, \mathcal{A})$ tal que $\left.g\right|_{\left(k Q_{\Lambda}\right)_{0}}=g_{0}$ e $\left.g\right|_{\left(k Q_{\Lambda}\right)_{1}}=g_{1}$. Resumidamente, $g$ é unicamente determinado pelas seguintes informações:

- $g(\gamma)=f_{i}(\gamma)$ para cada caminho $\gamma$ sobre $\Sigma_{i}$. (Observe que $\Sigma_{i}$ é uma subaljava plena de $Q_{\Lambda}$ ).

- $g(\alpha)=e_{i}^{l} \alpha e_{j}^{m}$ para cada flecha $\alpha: e_{i}^{l} \rightarrow e_{j}^{m}$ tal que $i \neq j$.

Não é difícil se convencer de que $g$ é um epimorfismo e que Ker $g=\Omega_{\Lambda}$. Dessa forma, pelo Primeiro Teorema do Isomorfismo, $g$ induz um isomofismo de álgebras $\tilde{g}: k Q_{\Lambda} / \Omega_{\Lambda} \rightarrow \Lambda$.

Conforme já antecipado acima, vamos estender o Teorema 4.2.3, de forma a contemplar não só as ACG's, como também as ACGR's. Primeiro precisamos introduzir algumas notações. Seja $\Gamma$ uma aljava acíclica. Para simplificar as notações, podemos assumir (sem perda de generalidade) que $\Gamma_{0}=\{1, \ldots, n\}$ e que $\Gamma_{1}=\left\{\alpha_{1}, \ldots, \alpha_{m}\right\}$. Seja $\mathcal{A}=\left\{A_{1}, \ldots, A_{n}\right\}$, onde $A_{i}=k \Sigma_{i} / \Omega_{i}$ é uma ACR para todo $i$, sendo $\Sigma_{i}$ uma aljava e $\Omega_{i}$ um ideal admissível de $k \Sigma_{i}$.

Recuperando a notação da demonstração do Teorema 4.2.3, $f_{i}: k \Sigma_{i} \rightarrow \frac{k \Sigma_{i}}{\Omega_{i}}$ será a projeção canônica para todo $i$. Também existe um epimorfismo de álgebras

$$
g: k Q \rightarrow k(\Gamma, \mathcal{A})
$$

onde $Q$ é a aljava obtida a partir de $\Gamma, \Sigma_{1}, \ldots, \Sigma_{n}$, tal como no enunciado do mesmo teorema. (A aljava $Q$ também é a aljava de Gabriel de $k(\Gamma, \mathcal{A})$, mas para simplificar a notação vamos denotar essa aljava apenas por $Q)$. Além disso, Ker $g=\Omega \doteq\left(\Omega_{1}, \ldots, \Omega_{n}\right)$. Definimos ainda $c_{i}$ como o número de vértices de $\Sigma_{i}$, e $c_{i j}=c_{i} . c_{j}$.

Agora rotulamos os vértices de $\Sigma_{i}$ da seguinte forma: $\left(\Sigma_{i}\right)_{0}=\left\{e_{i}^{1}, \ldots, e_{i}^{c_{i}}\right\}$. Novamente, lembrando a demonstração do Teorema de Gabriel 2.6.1, este conjunto pode ser entendido como um conjunto completo de idempotentes primitivos dois-a-dois ortogonais em $A_{i}$. Dessa forma, pelo Teorema 4.2.3, se $\alpha_{l}: i \rightarrow j$ é uma flecha de $\Gamma$, existem $c_{i j}$ flechas correspondentes em $Q$, as quais serão denotadas por $\alpha_{l p q}: e_{i}^{p} \rightarrow e_{j}^{q}$, com $1 \leq p \leq c_{i}, 1 \leq q \leq c_{j}$. Com essa notação,

$$
Q_{1}=\left(\Sigma_{1}\right)_{1} \cup \ldots \cup\left(\Sigma_{n}\right)_{1} \cup\left\{\alpha_{l p q}: 1 \leq l \leq m, 1 \leq p \leq s\left(\alpha_{l}\right), 1 \leq q \leq e\left(\alpha_{l}\right)\right\}
$$

Precisamos agora ver como serão as relações. Suponha que a aljava $\Gamma$ está equipada com um conjunto finito $I$ de relações. Então, seguindo a Definição 4.1.1, isso significa que vamos considerar o quociente de $k(\Gamma, \mathcal{A})$ pelo ideal gerado pelo conjunto $\mathcal{A}(I)$ abaixo: 


$$
\begin{aligned}
\mathcal{A}(I) \doteq & \left\{\sum_{1 \leq i \leq s} \lambda_{i} \beta_{i 1} \overline{\gamma_{i 1}} \beta_{i 2} \ldots \overline{\gamma_{i(r-1)}} \beta_{i r}:\right. \\
& \left.\gamma_{i j} \text { é um caminho em } \Sigma_{e\left(\beta_{i j}\right)}, \text { e } \sum_{1 \leq i \leq s} \lambda_{i} \beta_{i 1} \ldots \beta_{i r} \text { é uma relação de } I\right\}
\end{aligned}
$$

Defina ainda, em $k Q$,

$$
\begin{aligned}
R \doteq & \left\{\sum _ { 1 \leq i \leq s } \lambda _ { i } ( \beta _ { i 1 , e _ { l } ^ { p } , s ( \gamma _ { i 1 } ) } ) \gamma _ { i 1 } ( \beta _ { i 2 , e ( \gamma _ { i 1 } ) , s ( \gamma _ { i 2 } ) } ) \gamma _ { i 2 } \ldots \gamma _ { i ( r - 1 ) } \left(\beta_{\left.i r, e\left(\gamma_{i(r-1)}\right), e_{l^{\prime}}^{q}\right):}\right.\right. \\
& \forall i, \lambda_{i} \in k, \gamma_{i j} \text { é um caminho em } \Sigma_{e\left(\beta_{i j}\right)} \text { para } j \geq 1, \text { e } \sum_{1 \leq i \leq s} \lambda_{i} \beta_{i 1} \ldots \beta_{i r} \\
& \text { é uma relação em } \left.I \text { entre os vértices } l \text { e } l^{\prime}, 1 \leq p \leq c_{l}, 1 \leq q \leq c_{l^{\prime}}\right\}
\end{aligned}
$$

E seja $L$ o ideal gerado por $R$.

Lema 4.2.4. Com as notações acima, vale que $g(L)=(\mathcal{A}(I))$.

Demonstração. Tendo em mente como o epimorfismo $g$ foi definido na demonstração do Teorema 4.2.3, vale que:

$$
\begin{aligned}
& g\left(\sum_{1 \leq i \leq s} \lambda_{i}\left(\beta_{i 1, e_{l}^{p}, s\left(\gamma_{i 1}\right)}\right) \gamma_{i 1}\left(\beta_{i 2, e\left(\gamma_{i 1}\right), s\left(\gamma_{i 2}\right)}\right) \gamma_{i 2} \ldots \gamma_{i(r-1)}\left(\beta_{\left.i r, e\left(\gamma_{i(r-1)}\right), e_{l^{\prime}}^{q}\right)}\right)\right. \\
& =\sum_{1 \leq i \leq s} \lambda_{i} g\left(\beta_{i 1, e_{l}^{p}, s\left(\gamma_{i 1}\right)}\right) f_{e\left(\beta_{i 1}\right)}\left(\gamma_{i 1}\right) g\left(\beta_{i 2, e\left(\gamma_{i 1}\right), s\left(\gamma_{i 2}\right)}\right) f_{e\left(\beta_{i 2}\right)}\left(\gamma_{i 2}\right) \ldots \\
& f_{e\left(\beta_{i(r-1)}\right)}\left(\gamma_{i(r-1)}\right) g\left(\beta_{\left.i r, e\left(\gamma_{i(r-1)}\right), e_{l^{\prime}}^{q}\right)}\right. \\
& =\sum_{1 \leq i \leq s} \lambda_{i} e_{l}^{p} \beta_{i 1} e_{e\left(\beta_{i 1}\right)}^{s\left(\gamma_{i 1}\right)} \overline{\gamma_{i 1}} e_{s\left(\beta_{i 2}\right)}^{e\left(\gamma_{i 1}\right)} \beta_{i 2} e_{e\left(\beta_{i 2}\right)}^{s\left(\gamma_{i 2}\right)} \overline{\gamma_{i 2}} \ldots \overline{\gamma_{i(r-1)}} e_{s\left(\beta_{i r}\right)}^{e\left(\gamma_{i(r-1)}\right)} \beta_{i r} e_{l^{\prime}}^{q} \\
& =\sum_{1 \leq i \leq s} \lambda_{i} e_{l}^{p} \beta_{i 1} \overline{\gamma_{i 1}} \beta_{i 2} \overline{\gamma_{i 2}} \ldots \overline{\gamma_{i(r-1)}} \beta_{i r} e_{l^{\prime}}^{q} \\
& =e_{l}^{p}\left(\sum_{1 \leq i \leq s} \lambda_{i} e_{l}^{p} \beta_{i 1} \overline{\gamma_{i 1}} \beta_{i 2} \overline{\gamma_{i 2}} \ldots \overline{\gamma_{i(r-1)}} \beta_{i r}\right) e_{l^{\prime}}^{q}
\end{aligned}
$$

A primeira conclusão que tiramos do raciocínio acima é de que $g(R) \subseteq(\mathcal{A}(I))$. E como $g$ é um homomorfismo de álgebras, isso implica que $g(L) \subseteq(\mathcal{A}(I))$.

Para a recíproca, lembre que $\sum_{p} e_{l}^{p}=1_{k \Sigma_{l}}$ e que $\sum_{q} e_{l^{\prime}}^{q}=1_{k \Sigma_{l^{\prime}}}$. Com isto, o cálculo feito acima também mostra que $\mathcal{A}(I)$ está contido no ideal gerado por $g(R)$, e portanto no ideal gerado por $g(L)$, já que $R \subset L$. Mas de novo, $g$ é sobrejetor, logo pelo Teorema da Correspondência $g(L)$ já é um ideal. Isso implica que $(\mathcal{A}(I)) \subseteq g(L)$.

Teorema 4.2.5. Seja $k(\Gamma, \mathcal{A})$ uma álgebra de caminhos generalizada, onde $\Gamma$ é uma aljava acíclica com $\Gamma_{0}=\{1, \ldots, n\}$, e $\mathcal{A}=\left\{A_{1}, \ldots, A_{n}\right\}$ é uma família de álgebras, tal que, para cada $i \in \Gamma_{0}$, 
$A_{i}=k \Sigma_{i} / \Omega_{i}$, onde $\Sigma_{i}$ é uma aljava e $\Omega_{i}$ é um ideal admissível de $k \Sigma_{i}$. Sejam ainda $Q$ a aljava obtida a partir de $\Gamma, \Sigma_{1}, \ldots, \Sigma_{n}$ tal como no enunciado do Teorema 4.2.3, $\Omega=\left(\Omega_{1}, \ldots, \Omega_{n}\right), I$ um conjunto finito de realções sobre $\Gamma$ e sejam $\mathcal{A}(I) \subseteq k(\Gamma, \mathcal{A})$ tal como na Definição 4.1.1 e $L \subseteq k Q$ o ideal gerado pelo conjunto $R$ definido na equação 4.1 acima. Então temos que $\Omega+L$ é um ideal admissível de $k Q$ e que existe um isomorfismo:

$$
\frac{k Q}{\Omega+L} \cong \frac{k(\Gamma, \mathcal{A})}{(\mathcal{A}(I))} \doteq k(\Gamma, \mathcal{A}, I)
$$

Demonstração. Já sabemos que $\Omega$ é admissível pelo Teorema 4.2.3. Além disso $L$ é admissível porque é gerado por $R$, um conjunto de relações que são somas de caminhos de comprimento pelo menos dois, e assumimos que $\Gamma$ é acíclica e finita. Disso segue que $\Omega+L$ é admissível.

Denote $J=(\mathcal{A}(I))$. Seja

$$
\pi: k(\Gamma, \mathcal{A}) \rightarrow \frac{k(\Gamma, \mathcal{A})}{J}
$$

a projeção canônica. Defina

$$
\tilde{\phi} \doteq \pi \circ g: k Q \stackrel{g}{\rightarrow} k(\Gamma, \mathcal{A}) \stackrel{\pi}{\rightarrow} \frac{k(\Gamma, \mathcal{A})}{J}
$$

Como $\tilde{\phi}$ é um epimorfismo, pelo Primeiro Teorema do Isomorfismo ela induz um isomorfismo

$$
\phi: \frac{k Q}{\operatorname{Ker} \tilde{\phi}} \rightarrow \frac{k(\Gamma, \mathcal{A})}{J}
$$

Afirmamos que $\operatorname{Ker} \tilde{\phi}=g^{-1}(J)$. De fato,

$$
x \in \operatorname{Ker} \tilde{\phi} \Leftrightarrow \tilde{\phi}(x)=0 \Leftrightarrow \pi \circ g(x)=0 \Leftrightarrow g(x) \in J \Leftrightarrow x \in g^{-1}(J)
$$

Portanto resta provar que $g^{-1}(J)=\Omega+L$.

$(\supseteq)$ Como $\Omega=\operatorname{Ker} g, g(\Omega)=0 \subseteq J \Rightarrow \Omega \subseteq g^{-1}(J)$. Pelo Lema 4.2.4, $g(L)=(\mathcal{A}(I))=J$, e $\operatorname{assim} L \subseteq g^{-1}(J)$. Portanto $\Omega+L \subseteq g^{-1}(J)$, já que $g^{-1}(J)=\operatorname{Ker} \tilde{\phi}$ é um ideal e logo fechado para soma.

$(\subseteq)$ Seja $x \in g^{-1}(J)$. Então $g(x) \in J$ e, pelo Lema 4.2.4, existe um $l \in L$ tal que $g(x)=g(l)$. Daí $x-l \in \operatorname{Ker} g=\Omega$, e logo existe $\omega \in \Omega$ tal que $x-l=\omega$. Portanto $x=\omega+l$, com $\omega \in \Omega \mathrm{e}$ $l \in L$. Isso mostra que $x \in \Omega+L$.

Exemplo 4.2.6. Seja $\Lambda$ a ACGR dada pela aljava

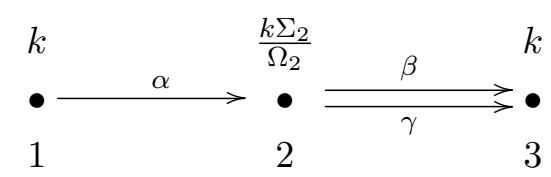

com uma relação $\alpha \beta=0$, onde $\Sigma_{2}$ é a aljava 


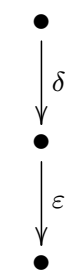

e $\Omega_{2}=(\delta \varepsilon)$. Aplicando o Teorema 4.2.5, concluímos que a aljava de Gabriel $Q$ de $\Lambda$ é dada por

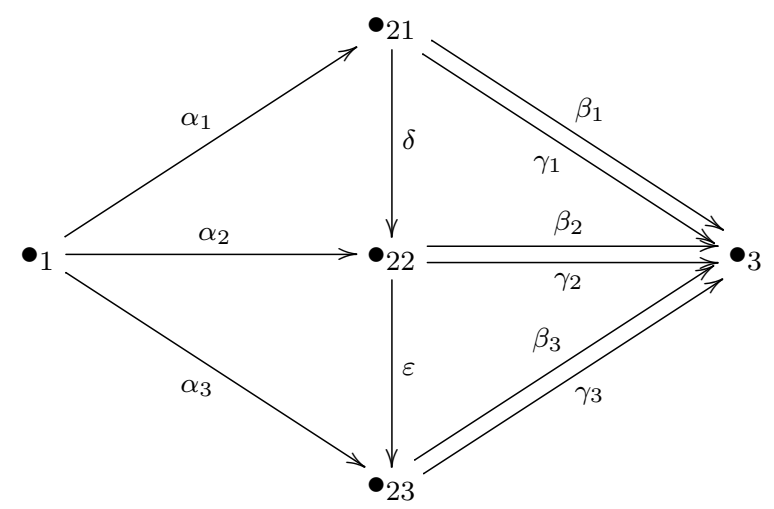

e que $\Lambda \cong k Q /(\Omega+L)$, onde $\Omega=(\delta \varepsilon)$ e

$$
L=\left(\alpha_{1} \beta_{1}, \alpha_{1} \delta \beta_{2}, \alpha_{1} \delta \varepsilon \beta_{3}, \alpha_{2} \beta_{2}, \alpha_{2} \epsilon \beta_{3}, \alpha_{3} \beta_{3}\right)
$$

\subsection{Simplificações}

Nesta seção nosso interesse está em obter consequências do Teorema 4.2.5. Aquele teorema mostrou como obter uma álgebra de caminhos com relações (ACR) isomorfa a uma ACGR dada. O que queremos fazer aqui é o processo inverso: decidir quando existe uma ACGR isomorfa a uma ACR dada. Ou seja, queremos realizar uma ACR como uma ACGR.

Observação 4.3.1. Para tornar as ideias mais claras, desde já citamos que sempre há duas maneiras de realizar uma ACR como uma ACGR, as quais serão chamadas de triviais. Seja $A$ uma álgebra. Então:

- Se $\Gamma=\bullet$, i.e., $\Gamma$ é uma aljava com apenas vértice e nenhuma flecha, e se $\mathcal{A}=\{A\}$, então obviamente $A \cong k(\Gamma, \mathcal{A})$.

- Pelo Teorema de Gabriel 2.6.1, existem uma aljava $Q$ e um ideal admissível $I$ de $k Q$ tais que $A=k Q / I$. Seja $\mathcal{A}=\left\{k: i \in Q_{0}\right\}$. Então claramente $A \cong k(Q, \mathcal{A}, I)$.

Definição 4.3.2. Seja $A$ uma álgebra. Dizemos que uma $\operatorname{ACGR~} k(\Gamma, \mathcal{A}, I)$ é uma simplificação de $A$ se $A \cong k(\Gamma, \mathcal{A}, I)$. As duas simplificações acima são as simplificações triviais. Se $I=0$, dizemos que a simplificação é sem relações. Se $\Gamma$ é acíclica, dizemos que a simplificação é sem ciclos.

Definição 4.3.3. Sejam $k(\Gamma, \mathcal{A}, I)$ e $k(\Delta, \mathcal{B}, J)$ duas simplificações de $A$, com $\mathcal{A}=\left\{A_{i}: i \in \Gamma_{0}\right\}$ e $\mathcal{B}=\left\{B_{i}: i \in \Delta_{0}\right\}$. Dizemos que elas são equivalentes se existir um isomorfismo de aljavas (ou 
seja, com a terminologia da Seção 2.4, um morfismo de aljavas que tem inverso) $\phi: \Gamma \rightarrow \Delta$ tal que $A_{i} \cong B_{\phi(i)}$ para cada $i \in \Gamma_{0}$ e se existir um isomorfismo de álgebras $k \Gamma / I \cong k \Delta / J$.

Definição 4.3.4. Dizemos que uma álgebra $A$ é simplificável se ela admite uma simplificação que não é equivalente a uma das duas simplificações triviais listadas em 4.3.1. Também usamos os termos simplificável sem relações ou sem ciclos no caso em que essa simplificação não-trivial é sem relações ou sem ciclos respectivamente.

Um exemplo de álgebra simplificável foi dado no Exemplo 4.2.6, sendo a versão ACGR da álgebra $\Lambda$ a simplificação não-trivial. (Para ver que é não-trivial, basta observar que o número de vértices é diferente do número das simplificações triviais). Neste caso, a simplificação é ainda sem ciclos.

\subsection{Um critério para simplificabilidade}

O objetivo desta seção é fornecer um critério para decidir se uma álgebra é ou não é simplificável. Porém, como os Teoremas 4.2.3 e 4.2.5 só tratam do caso em que $\Gamma$ é acíclica, nos limitamos a analisar apenas as simplificações sem ciclos.

\subsubsection{Relações de equivalência sobre vértices}

Vamos primeiro estabelecer algumas definições úteis:

Definição 4.4.1. Sejam $Q$ uma aljava e $\sim \in Q_{0} \times Q_{0}$ uma relação de equivalência sobre os vértices de $Q$.

- A aljava reduzida de $Q$ por $\sim$, denotada por $Q^{\sim}$, é definida como a subaljava obtida de $Q$ ao deletar todas as flechas contidas em uma classe de equivalência. Ou seja, $\left(Q^{\sim}\right)_{0}=Q_{0}$ e $\left(Q^{\sim}\right)_{1}=\left\{\alpha \in Q_{1}: s(\alpha) \nsim e(\alpha)\right\}$.

- Dizemos que $\sim$ é coerente com as flechas de $Q$ se:

1. Para cada flecha da forma $i \rightarrow j$ contida em um ciclo orientado de $Q$, temos que $i \sim j$.

2. Se $i \sim j$ e $j \nsim k$, então $[i, k]=[j, k]$ e $[k, i]=[k, j]$.

- Suponha que $\sim$ é coerente com as flechas. Então uma rotulagem de $Q$ será um morfismo de álgebras $z: Q^{\sim} \rightarrow \frac{Q^{\sim}}{\sim}$ tal que $z(x)=\bar{x}$ para cada vértice $x \in Q_{0}$, e tal que, para cada par de vértices $x, y \in Q_{0}$, a restrição $\left.z\right|_{Q^{\sim}(x, y)} Q^{\sim}(x, y) \rightarrow \frac{Q^{\sim}}{\sim}(x, y)$ é bijetora. (Como $\sim$ é coerente, uma rotulagem $z$ com essa forma vai sempre existir). No resto desta definição, sempre assumimos que $Q$ tem uma rotulagem denotada por $z$.

- Seja $\gamma$ um caminho ordinário sobre $Q$. Então sempre é possível escrever $\gamma=\delta_{0} \alpha_{1} \delta_{1} \ldots \alpha_{m} \delta_{m}$, onde, para cada $i, \delta_{i}$ é um caminho cujos vértices estão todos identificados por $\sim$, e $\alpha_{i}: j_{1} \rightarrow j_{2}$ é uma flecha tal que $j_{1} \nsim j_{2}$. Daí o caminho induzido por $\gamma$ na aljava quociente é definido como sendo o caminho $z(\gamma)=z\left(\alpha_{1}\right) z\left(\alpha_{2}\right) \ldots z\left(\alpha_{m}\right)$.

- Seja $\gamma=\sum_{t=1}^{r} \lambda_{t} \gamma_{t}$ uma relação sobre $Q$, com $\lambda_{t} \in k$ e $\gamma_{t}$ um caminho sobre $Q$ para todo $t$, sendo os caminhos $\gamma_{t}$ dois a dois distintos. 
1. Dizemos que $\gamma$ é uma relação interna se $l\left(z\left(\gamma_{t}\right)\right)=0$ para todo $t$.

2. Se $\gamma$ não é interna e $z\left(\gamma_{t}\right) \neq z\left(\gamma_{s}\right)$ quando $t$ e $s$ são distintos, dizemos que $\gamma$ é uma relação externa .

- Seja $R$ um conjunto finito de relações sobre $Q$. Dizemos que $R$ e $\sim$ são compatíveis se:

1. Toda relação em $R$ é ou interna ou externa em relação a $\sim$.

2. Se $\bar{x} \subseteq Q_{0}$ é uma classe de equivalência em relação a $\sim$ e $\Sigma_{\bar{x}}$ denota a subaljava plena de $Q_{0}$ determinada por $\bar{x}$, então as relações internas contidas em $\bar{x}$ geram um ideal admissível em $k \Sigma_{\bar{x}}$.

3. Se $\gamma=\sum_{t=1}^{r} \lambda_{t} \gamma_{t} \in R$ é externa, onde $\lambda_{t} \in k$ e $\gamma_{t}$ é um caminho sobre $Q$ para todo $t$, então para toda família de caminhos $\left\{\eta_{t}: 1 \leq t \leq r\right\}$ sobre $Q$ tal que para cada $t$, $z\left(\gamma_{t}\right)=z\left(\eta_{t}\right)$, deve valer que $\sum_{t=1}^{r} \lambda_{t} \eta_{t} \in R$.

Exemplo 4.4.2. Seja $A$ a ACR dada pela aljava $Q$ abaixo:

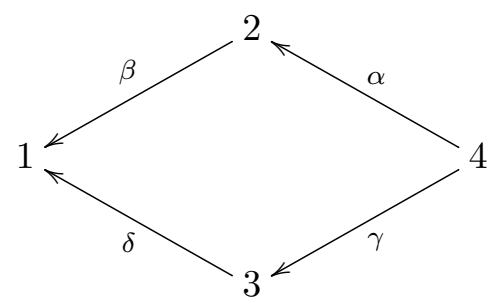

tendo um conjunto de relações $R$. Seja $\sim$ a menor relação de equivalência sobre $Q_{0}$ tal que $2 \sim 3$. Então $\sim$ é coerente com as flechas de $Q$. O quociente é dado por:

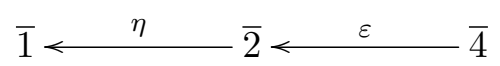

Neste caso, só existe uma única rotulagem $z$ de $Q$. Seja $R=\{\alpha \beta-\gamma \delta\}$. Temos que $z(\alpha \beta)=$ $z(\gamma \delta)=\varepsilon \eta$. Isso significa que $\alpha \beta-\gamma \delta$ não é nem interna nem externa, e portanto $R$ não é compatível com $\sim$.

No entanto, é fácil verificar que $R=\{\alpha \beta, \gamma \delta\}$ é compatível com $\sim$. Este conjunto possui duas relações externas.

Observação 4.4.3. Note que não há ambiguidade na definição de compatibilidade, pois a condição dada é sobre o conjunto de geradores e não sobre o que eles geram.

A necessidade de rotular as aljavas reside no fato de elas poderem ter flechas múltiplas entre dois vértices. Se uma aljava não tem flechas múltiplas, então só há uma rotulagem possível e portanto a noção de compatibilidade depende apenas do conjunto de relações.

Veremos abaixo (Teoremas 4.4.4 e 4.4.5) que há uma certa conexão entre simplificações e relações de equivalência compatíveis. A grosso modo, é possível obter uma através da outra. Analisar esta conexão é o que nos levará ao critério de simplificabilidade prometido acima. Este estudo será dividido nas duas subseções abaixo. 


\subsubsection{Simplificações a partir de relações de equivalência}

Começamos com uma álgebra $A=k Q /(R)$, onde $Q$ é uma aljava e $R$ é um conjunto finito de relações sobre $Q$ tal que $(R)$ é admissível. Consideramos uma relação de equivalência $\sim \subseteq Q_{0} \times Q_{0}$, coerente com as flechas de $Q$ e compatível com $R$ em relação a uma rotulagem $z$ de $Q$. Nosso objetivo é produzir uma simplificação sem $\operatorname{ciclos} k(\Gamma, \mathcal{A}, L)$ de $A$.

A aljava $\Gamma$ será a aljava quociente $\Gamma \doteq \frac{Q^{\sim}}{\sim}$, e portanto a rotulagem $z$ é um morfismo de aljavas $z: Q^{\sim} \rightarrow \Gamma$ que induz bijeções $Q^{\sim}(x, y) \leftrightarrow \Gamma(x, y)$ para cada par de vértices $x, y \in Q_{0}$ tal que $x \nsim y$.

Observamos que $\Gamma$ definida deste modo é acíclica; de fato, se $\Gamma$ contém um ciclo orientado, então este precisa vir de um ciclo orientado de $Q$, o que é absurdo, já que todo ciclo orientado de $Q$ está contido em uma mesma classe de equivalência, por força da Definição 4.4.1.

Agora escreva $\Gamma_{0}=\left\{\overline{x_{1}}, \ldots, \overline{x_{n}}\right\}$, onde os $x_{i} \in Q_{0}$ estão todos em classes de equivalência distintas. Denotamos por $\Sigma_{i}$ a subaljava plena de $Q$ cujos vértices são aqueles de $\overline{x_{i}}$.

Pela nossa hipótese, podemos escrever $R=R^{\text {int }} \sqcup R^{\text {ext }}$, onde $R^{\text {int }}$ é composto apenas por relações internas, e $R^{\text {ext }}$, por externas. Além disso, $R^{\text {int }}=R_{1} \sqcup \ldots \sqcup R_{n}$, onde $R_{i}$ denota o conjunto de relações internas contidas em $\Sigma_{i}$, para cada $i$. Note que, pela Definição $4.4 .1, R_{i}$ gera um ideal admissível em $\Sigma_{i}$.

Definimos portanto $A_{i}=k \Sigma_{i} / R_{i}$ e $\mathcal{A}=\left\{A_{1}, \ldots, A_{n}\right\}$.

Seja $\gamma \in R^{e x t}$. Podemos então escrever

$$
\gamma=\sum_{t=1}^{r} \lambda_{t} \delta_{t 0} \alpha_{t 1} \delta_{t 1} \ldots \alpha_{t n_{t}} \delta_{t n_{t}}
$$

onde, para cada $t$ e $i, \lambda_{t} \in k, \delta_{t i}$ é um caminho cujos vértices estão todos em uma mesma classe de equivalência, e $\alpha_{t i}$ é uma flecha entre vértices que pertencem a classes de equivalência diferentes. Daí definimos uma relação sobre $\Gamma$ da seguinte forma:

$$
z(\gamma) \doteq \sum_{t=1}^{r} \lambda_{t} z\left(\delta_{t 0} \alpha_{t 1} \delta_{t 1} \ldots \alpha_{t n_{t}} \delta_{t n_{t}}\right)=\sum_{t=1}^{r} \lambda_{t} z\left(\alpha_{t 1}\right) \ldots z\left(\alpha_{t n_{t}}\right)
$$

Finalmente defina $L=\left\{z(\gamma): \gamma \in R^{e x t}\right\}$.

Resta provar que esta é de fato uma simplificação de $A$, isto é, devemos provar que $A=$ $k Q /(R) \cong k(\Gamma, \mathcal{A}, L)$.

Se aplicarmos o Teorema 4.2.5 à ACGR $k(\Gamma, \mathcal{A}, L)$, obtemos uma aljava que facilmente se constata ser isomorfa à aljava $Q$. Também se obtém um conjunto de relações $M$ sobre $Q$ que facilmente se verifica ser igual a $R^{\text {int }} \bigsqcup N$, onde $N$ é um conjunto de relações obtido de $L$. Resumindo, nós temos

$$
k(\Gamma, \mathcal{A}, L) \cong \frac{k Q}{(M)}=\frac{k Q}{\left(R^{\text {int }} \bigsqcup N\right)}, \mathrm{e} \frac{k Q}{(R)}=\frac{k Q}{\left(R^{\text {int }} \bigsqcup R^{\text {ext }}\right)}
$$

Portanto resta provar que $R^{e x t}=N$.

$(\subseteq)$ Seja

$$
\gamma=\sum_{t=1}^{r} \lambda_{t} \delta_{t 0} \alpha_{t 1} \delta_{t 1} \ldots \alpha_{t n_{t}} \delta_{t n_{t}} \in R^{e x t}
$$


onde usamos a mesma notação de antes. Então

$$
z(\gamma)=\sum_{t=1}^{r} \lambda_{t} z\left(\alpha_{t 1}\right) \ldots z\left(\alpha_{t n_{t}}\right) \in L
$$

Também vale que $\delta_{t 0}$ é um caminho em $\Sigma_{s\left(\alpha_{t 1}\right)}$ e que $\delta_{t i}$ é um caminho em $\Sigma_{e\left(\alpha_{t i}\right)}$ para todos $i>0$. Consequentemente, pelo Teorema 4.2.5,

$$
\gamma=\sum_{t=1}^{r} \lambda_{t} \delta_{t 0} \alpha_{t 1} \delta_{t 1} \ldots \alpha_{t n_{t}} \delta_{t n_{t}} \in N
$$

() Lembramos novamente do Teorema 4.2.5. Todo elemento de $N$ tem forma

$$
\gamma=\sum_{t=1}^{r} \lambda_{t} \eta_{t 0} \alpha_{t 1} \eta_{t 1} \ldots \alpha_{t n_{t}} \eta_{t n_{t}}
$$

onde $\lambda_{t} \in k, \eta_{t 0}$ é um caminho sobre $\Sigma_{s\left(\alpha_{t 1}\right)}, \eta_{t i}$ é um caminho sobre $\Sigma_{e\left(\alpha_{t i}\right)}$ para cada $i>0$, e

$$
\sum_{t=1}^{r} \lambda_{t} z\left(\alpha_{t 1}\right) \ldots z\left(\alpha_{t n_{t}}\right)
$$

é uma relação em $L$. Mas, pela definição de $L$, isso significa que temos uma relação

$$
\gamma^{\prime}=\sum_{t=1}^{r} \lambda_{t} \delta_{t 0} \beta_{t 1} \delta_{t 1} \ldots \beta_{t n_{t}} \delta_{t n_{t}} \in R^{e x t}
$$

onde $\delta_{t 0}$ é um caminho sobre $\Sigma_{s\left(\alpha_{t 1}\right)}, \delta_{t i}$ é um caminho sobre $\Sigma_{e\left(\alpha_{t i}\right)}$ para cada $i>0$, e para cada $t, i, z\left(\beta_{t i}\right)=z\left(\alpha_{t i}\right)$.

Como, para cada $t$, os $t$-ésimos termos da soma em $\gamma$ e $\gamma^{\prime}$ têm ambos a mesma imagem via $z$, $\gamma^{\prime} \in R^{e x t}$ e $R$ é compatível com $\sim$, temos que $\gamma \in R^{e x t}$. Isso conclui a demonstração.

Para concluir a presente subseção, vamos resumir nossas conclusões sob o enunciado abaixo:

Teorema 4.4.4. Seja $A=k Q /(R)$ uma álgebra, onde $Q$ é uma aljava com uma rotulagem $z$ fixada e $R$ é um conjunto finito de relações sobre $Q$ tal que $(R)$ é admissível. Suponha que $\sim \subseteq Q_{0} \times Q_{0}$ é uma relação de equivalência coerente com as flechas de $Q$ e compatível com $R$. Então temos uma simplificação sem ciclos $k(\Gamma, \mathcal{A}, L)$ de $A$, que é definida da seguinte forma:

- $\Gamma_{0}=Q_{0} / \sim$

- Se $\bar{x}, \bar{y} \in \Gamma_{0}$, então $[\bar{x}, \bar{y}]_{\Gamma}= \begin{cases}{[x, y]_{Q}} & \text { if } x \nsim y \\ 0 & \text { if } x \sim y\end{cases}$

- $\mathcal{A}=\left\{A_{\bar{x}}: \bar{x} \in \Gamma_{0}\right\}$, onde $A_{\bar{x}}$ é a álgebra definida como $k \Sigma_{\bar{x}} / R_{\bar{x}}$, onde $\Sigma_{\bar{x}}$ é a subaljava plena de $Q$ cujos vértices são os de $\bar{x}$ e $R_{\bar{x}}$ é o subconjunto de $R$ das relações contidas em $\Sigma_{\bar{x}}$.

- $L=\left\{z(\gamma): \gamma \in R \backslash\left(\cup_{\left.\bar{x} \in \Gamma_{0}\right)} R_{\bar{x}}\right\}\right.$.

\subsubsection{Relações de equivalência a partir de simplificações}

Começamos com uma álgebra $A=k Q /(R)$, onde $Q$ é uma aljava, $R$ é um conjunto finito de relações sobre $Q$ tal que $(R)$ é admissível, e com uma simplificação sem ciclos $k(\Gamma, \mathcal{A}, L)$. O objetivo 
é construir uma relação de equivalência $\sim \subseteq Q_{0} \times Q_{0}$ coerente com as flechas de $Q$, uma rotulagem de $Q$, e um conjunto finito de relações $R^{\prime}$ sobre $Q$ compatível com $\sim$ tal que $\left(R^{\prime}\right)$ é admissível e $k Q /(R) \cong k Q /\left(R^{\prime}\right)$.

Novamente podemos supor que $\Gamma_{0}=\{1, \ldots, n\}$ e denotamos $\mathcal{A}=\left\{A_{1}, \ldots, A_{n}\right\}$, onde $A_{i}=$ $k \Sigma_{i} /\left(R_{i}\right)$ para todo $i$, sendo $\Sigma_{i}$ uma aljava e $R_{i}$ um conjunto finito de relações sobre $\Sigma_{i}$ tal que $\left(R_{i}\right)$ é admissível. Aplicando o Teorema 4.2.5 à ACGR $k(\Gamma, \mathcal{A}, L)$, obtemos uma aljava que é igual a $Q$ por causa da unicidade no Teorema de Gabriel. Assim $Q_{0}=\bigsqcup_{i=1}^{n}\left(\Sigma_{i}\right)_{0}$. Como essa união é disjunta, ela é uma partição de $Q_{0}$ e portanto define uma relação de equivalência $\sim \subseteq Q_{0} \times Q_{0}$. Recuperando a notação da Seção 4.2, existe uma rotulagem $z: Q^{\sim} \rightarrow \frac{Q^{\sim}}{\sim}$ tal que $z\left(\alpha_{l p q}\right)=\alpha_{l}$ para cada $\alpha_{l}: i \rightarrow j \in \Gamma_{1}, 1 \leq p \leq c_{i}$, e $1 \leq q \leq c_{j}$. Também do Teorema 4.2.5, nós obtemos um conjunto finito $R^{\prime}$ de relações sobre $Q$ tal que $\left(R^{\prime}\right)$ é admissível e $k(\Gamma, \mathcal{A}, L) \cong k Q /\left(R^{\prime}\right)$. Como por hipótese $k Q /(R) \cong k(\Gamma, \mathcal{A}, L)$, temos que $k Q /(R) \cong k Q /\left(R^{\prime}\right)$. Resta portanto verificar os seguintes itens:

- é coerente com as flechas de $Q$ :

Como $\Gamma$ é acíclica, todos os vértices de um eventual ciclo orientado de $Q$ são identificados por $\sim$. Vejamos a segunda condição.

Fixe $x, y, z \in Q_{0} \operatorname{com} x \sim y, y \nsim z$. Daí

$$
[x, z]_{Q}=[\bar{x}, \bar{z}]_{\Gamma}=[\bar{y}, \bar{z}]_{\Gamma}=[y, z]_{Q}
$$

A verificação de que $[z, x]_{Q}=[z, y]_{Q}$ é análoga.

- $R^{\prime}$ é compatível com :

Seja

$$
\gamma=\sum_{t=1}^{r} \lambda_{t} \delta_{t 0} \alpha_{t 1} \delta_{t 1} \ldots \alpha_{t n_{t}} \delta_{t n_{t}} \in R^{\prime}
$$

onde mantemos a notação utilizada na Subseção 4.4.2. Se $n_{t}=0$ para todo $t$, então $\gamma$ é interna, ok. Suponha que isto não acontece. Como $\gamma \in R^{\prime}$, precisa valer que

$$
\sum_{t=1}^{r} \lambda_{t} z\left(\alpha_{t 1}\right) \ldots z\left(\alpha_{t n_{t}}\right)
$$

é uma relação em $L$, onde os caminhos $z\left(\alpha_{t 1}\right) \ldots z\left(\alpha_{t n_{t}}\right)$ podem ser assumidos como dois a dois distintos (já que sempre podemos denotar as relações de $L$ de uma maneira em que isto seja válido). Mas isso simplesmente significa que a relação $\gamma$ é externa.

Para todo $i$ entre 1 e $n$, o fato de que $R_{i}$ gera um ideal admissível em $k \Sigma_{i}$ vem das próprias definições. A última condição na Definição 4.4.1 é verificada ao se observar que o conjunto de relações externas em $R^{\prime}$ é igual ao conjunto

$$
\left\{\sum_{t=1}^{r} \lambda_{t} \gamma_{t}: \sum_{t=1}^{r} \lambda_{t} z\left(\gamma_{t}\right) \text { é uma relação em } L\right\}
$$

Este fato, por sua vez, é uma consequência direta da forma como $R^{\prime}$ foi obtido a partir de $L$. 
(Note que, também pela maneira como $R^{\prime}$ está definido, os caminhos $\delta_{t 0}$ e $\delta_{t n_{t}}$ acima serão sempre caminhos de comprimento zero.

Tal como fizemos na Subseção 4.4.2, vamos resumir nossa discussão com o seguinte teorema:

Teorema 4.4.5. Seja $A=k Q /(R)$ uma álgebra, onde $Q$ é uma aljava e $R$ é o um conjunto finito de relações sobre $Q$. Suponha que $A$ tem uma simplificação sem $\operatorname{ciclos} k(\Gamma, \mathcal{A}, L), \operatorname{com} \Gamma_{0}=\{1, \ldots, n\}$ e com $A_{i}=k \Sigma_{i} /\left(R_{i}\right)$ para todo $i$, sendo $\Sigma_{i}$ uma aljava e $R_{i}$ um conjunto de relações sobre $\Sigma_{i}$ tal que $\left(R_{i}\right)$ é admissível. Então $Q_{0}=\sqcup_{i=1}^{n}\left(\Sigma_{i}\right)_{0}$ e esta partição de $Q_{0}$ define uma relação de equivalência $\sim$ sobre $Q_{0}$ que é coerente com as flechas de $Q$. Além disso, existe um conjunto finito de relações $R^{\prime}$ sobre $Q$ obtido a partir do Teorema 4.2.5 tal que $\left(R^{\prime}\right)$ é admissível e vale o isomorfismo $k(\Gamma, \mathcal{A}, L) \cong k Q /\left(R^{\prime}\right)$, e existe uma rotulagem $z$ de $Q$ tal que $R^{\prime}$ é compatível (em relação a $z$ ) com a relação de equivalência $\sim$.

\subsection{Exemplos e aplicações}

Vamos dedicar este espaço para discutir algumas aplicações dos critérios discutidos na Seção 4.4, e também mostrar alguns exemplos práticos.

Observação 4.5.1. Seja $A$ uma álgebra, e suponha que $A=k Q /(R)$, onde $Q$ é a aljava de Gabriel de $A$ e $R$ é um conjunto finito de relações sobre $Q$. Se tomamos $\sim \subseteq Q_{0} \times Q_{0}$ como sendo o próprio conjunto $Q_{0} \times Q_{0}$ e aplicamos o Teorema 4.4.4, nós obtemos uma das simplificações triviais apresentadas na Observação 4.3.1. Se além disso $Q$ não tiver loops, então considerar $\sim=\{(x, x)$ : $\left.x \in Q_{0}\right\}$ nos levará à outra simplificação trivial.

O próximo corolário é inspirado na ideia presente em [11], p. 119. Na referência citada, o autor usa ACG's para eliminar loops da aljava de Gabriel de uma álgebra, porém sem usar a terminologia introduzida aqui.

Corolário 4.5.2. Seja $A=k Q /(R)$, onde $Q$ é uma aljava e $R$ é um conjunto finito de relações, sendo que cada relação de $R$ está contida em algum loop de $Q$. Com essa hipótese, podemos escrever $R=\cup_{i \in \Gamma_{0}} R_{i}$, onde $R_{i}$ é o conjunto de relações de $R$ que envolvem apenas o vértice $i$, e definir $\Sigma_{i}$ como a subaljava plena de $Q$ determinada pelo vértice $i$. Então $A$ tem uma simplificação sem relações $k(\Gamma, \mathcal{A})$, onde $\Gamma$ é a aljava obtida de $Q$ ao deletar todos os loops (isto é, $\Gamma_{0}=Q_{0}$ e $\left.\Gamma_{1}=Q_{1} \backslash \cup_{i \in Q_{0}}\left(\Sigma_{i}\right)_{1}\right)$, e $\mathcal{A}=\left\{k \Sigma_{i} / R_{i}: i \in \Gamma_{0}\right\}$. Além disso, $\Gamma$ tem o mesmo número de vértices de $Q$, e a simplificação que obtemos só poderá ser trivial quando $Q$ tiver apenas um vértice ou se não tiver loops.

Demonstração. Seja $\sim$ a relação de igualdade em $Q_{0}$, ou seja, a relação na qual todo vértice é identificado apenas consigo mesmo. De novo, esta relação de equivalência é coerente com as flechas e é compatível com $R$, sendo todas as relações internas. Daí as afirmações do enunciado seguem aplicando o Teorema 4.4.4.

Exemplo 4.5.3. Vamos analisar uma variação do Exemplo 3.1.2, discutido anteriormente. Considere a álgebra de caminhos sobre a aljava 


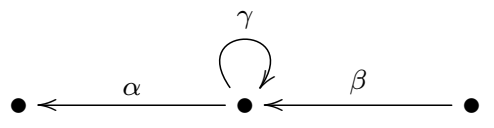

com a relação $\gamma^{n}=0$, onde $n$ é um número natural fixado. Aplicando o Corolário 4.5.2, esta álgebra tem uma simplificação, que é dada pela ACG

$$
k<\quad \alpha[t] /\left(t^{n}\right)<\beta \quad k
$$

Comparando com o Exemplo 3.1.2, aqui a álgebra que aparece no vértice do meio é $k[t] /\left(t^{n}\right)$, em vez de $k[t]$ como aparece naquele exemplo. Cabe lembrar, porém, que não devemos aplicar diretamente o Corolário 4.5.2 no Exemplo 3.1.2, já que $k[t]$ tem dimensão infinita, o que viola a suposição implícita que aqui fazemos de que as álgebras sempre satisfazem o Teorema de Gabriel.

Exemplo 4.5.4. Recuperemos a álgebra do Exemplo 4.4.2. Usando o Teorema 4.4.4, se $R=$ $\{\alpha \beta, \gamma \delta\}$ então a álgebra tem uma simplificação

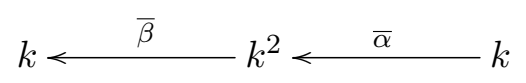

com a relação $\bar{\alpha} \bar{\beta}=0$.

Exemplo 4.5.5. Considere a álgebra dada pela aljava

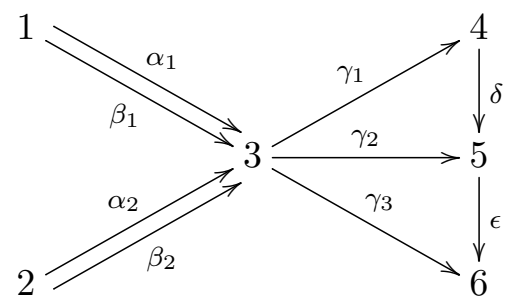

com relações $\alpha_{1} \gamma_{1}=0, \alpha_{1} \gamma_{2}=0, \alpha_{1} \gamma_{3}=0, \alpha_{2} \gamma_{1}=0, \alpha_{2} \gamma_{2}=0, \alpha_{2} \gamma_{3}=0$, e $\delta \epsilon=0$.

Seja $\sim$ a menor relação de equivalência tal que $1 \sim 2$ e $4 \sim 5 \sim 6$. Então $\sim$ é coerente com as flechas da aljava. O quociente da aljava reduzida por $\sim$ é dado por:

$$
a \stackrel{\alpha}{\beta} b \underset{\gamma}{\longrightarrow} c
$$

Então podemos definir uma rotulagem $z$ estabelecendo que $z(1)=z(2)=a, z(3)=b, z(4)=$ $z(5)=z(6)=c, z\left(\alpha_{1}\right)=z\left(\alpha_{2}\right)=\alpha, z\left(\beta_{1}\right)=z\left(\beta_{2}\right)=\beta$ e $z\left(\gamma_{1}\right)=z\left(\gamma_{2}\right)=z\left(\gamma_{3}\right)=\gamma$.

Com esta rotulagem, o conjunto de relações acima é compatível com $\sim$. De fato, $\delta \epsilon$ é uma relação interna e as relações da forma $\alpha_{i} \gamma_{j}$ são relações externas, todas induzindo o mesmo caminho $\alpha \gamma$ no quociente. Além disso, todo caminho cujo caminho induzido é $\alpha \gamma$ é um dos $\alpha_{i} \gamma_{j}$. Portanto esta álgebra é isomorfa à ACGR

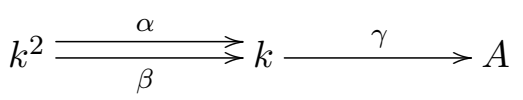

com a relação $\alpha \gamma=0$, onde $A$ é a álgebra dada pela aljava 


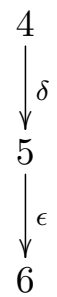

com a relação $\delta \epsilon=0$.

Exemplo 4.5.6. Neste exemplo vamos discutir uma conexão entre as ACGs e o conceito de blowingup, introduzido por Thomas Brüstle em [5].

Seja $\Gamma$ uma aljava e seja $I$ um ideal admissível de $k \Gamma$. Denote $A=k \Gamma / I$ e seja $v$ um vértice de $\Gamma$. Considere ainda um conjunto finito $F$. Então o blowing-up de $A$ via $F$ sobre o vértice $v$ é a ACR $k \tilde{\Gamma} / \tilde{I}$, onde $\tilde{\Gamma}$ e $\tilde{I}$ são definidos abaixo:

- A aljava $\tilde{\Gamma}$ é obtida de $\Gamma$ trocando o vértice $v$ pelos elementos de $F$. Além disso, toda flecha da forma $\alpha: v \rightarrow x$ dá origem a uma família de flechas da forma $\alpha_{f}: f \rightarrow x$, para todo $f \in F$. Vale o dual para as flechas que terminam em $v$. As flechas que não envolvem $v$ são mantidas.

- Note que existe um epimorfismo de aljavas $\tilde{\Gamma} \rightarrow \Gamma$, que por sua vez induz um epimorfismo de álgebras $\pi: k \tilde{\Gamma} \rightarrow k \Gamma$. Daí $\tilde{I}$ é definido como sendo a imagem inversa de $I$ via $\pi$.

Segue um exemplo prático: considere a aljava

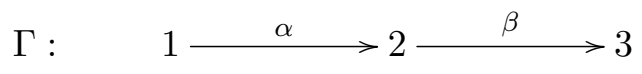

e considere $I=0$. Então o blowing-up de $k \Gamma / I$ via o conjunto $F=\{a, b\}$ sobre o vértice 2 é a álgebra $k \tilde{\Gamma} / \tilde{I}$, onde

$\tilde{\Gamma}:$

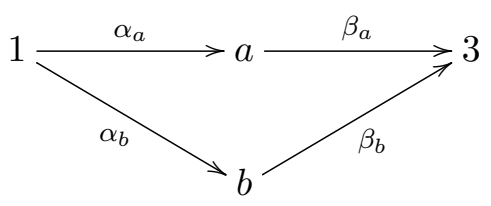

e $\tilde{I}=\left(\alpha_{a} \beta_{a}-\alpha_{b} \beta_{b}\right)$.

Em geral, usando o Teorema 4.2.5, temos que $k \tilde{\Gamma}$ é isomorfa à ACG $k(\Gamma, \mathcal{A})$, onde $\mathcal{A}=\left\{A_{i}\right.$ : $\left.i \in \Gamma_{0}\right\}$ é tal que

$$
A_{i}= \begin{cases}k, & \text { se } i \neq v \\ k^{F}, & \text { se } i=v\end{cases}
$$

Mas nem sempre vai valer que $k \tilde{\Gamma} / \tilde{I} \cong k(\Gamma, \mathcal{A}, I)$. O exemplo acima é uma prova disso, porque pelo Teorema $4.2 .5, k(\Gamma, \mathcal{A}, I)=k(\Gamma, \mathcal{A}) \cong k \tilde{\Gamma} \not k \tilde{\Gamma} / \tilde{I}$

No entanto, quando as relações sobre $\Gamma$ são todas monomiais (isto é, da forma $\gamma=0$, onde $\gamma$ é um caminho), então vale que $k \tilde{\Gamma} / \tilde{I} \cong k(\Gamma, \mathcal{A}, I)$. Isto porque as relações que obtemos pelo Teorema 4.2.5 geram os elementos que estão na imagem inversa via $\pi$ de cada relação monomial. 


\section{Capítulo 5}

\section{Representações de Álgebras de Caminhos Generalizadas}

Nosso interesse agora se volta ao estudo da categoria dos módulos sobre uma álgebra de caminhos generalizada. Quando consideramos álgebras de caminhos, o Teorema 2.6.5 de Gabriel permite trocar o estudo dos módulos pelo estudo das representações sobre as aljavas correspondentes. Para ACG's, precisaremos de um resultado análogo, como por exemplo o Teorema 2.4 de [6]. Vamos apresentar abaixo uma versão generalizada deste último resultado, que inclui também o caso em que há relações na aljava.

A ideia é estudar os módulos sobre $k(\Gamma, \mathcal{A})$ a partir dos módulos sobre as álgebras que pertencem a $\mathcal{A}$. Nesse sentido, queremos descrever módulos simples, projetivos e injetivos.

Daqui para frente, $\Gamma$ sempre denotará uma aljava, $\mathcal{A}=\left\{A_{i}: i \in \Gamma_{0}\right\}$ denotará uma família de álgebras, e $I$ denotará um conjunto de relações em $\Gamma$. Graças ao Teorema de Gabriel, podemos ainda supor que cada $A_{i}$ é da forma $k \Sigma_{i} / \Omega_{i}$, onde $\Sigma_{i}$ é uma aljava e $\Omega_{i}$ é um ideal admissível de $k \Sigma_{i}$. Vamos ainda denotar a ACGR correspondente por $\Lambda=k(\Gamma, \mathcal{A}, I)$. Por simplicidade, vamos denotar o elemento unidade das álgebras $A_{i}$ por $1_{i}$ em vez de $1_{A_{i}}$.

\subsection{Equivalência entre módulos e representações}

O objetivo desta Seção é provar o Teorema 5.1.3 abaixo, que é uma generalização do Teorema 2.4 de [6]. Como já observado acima, este vai ser um resultado essencial aqui, às vezes sendo usado sem necessidade de menção explícita.

Inspirando-se em [6], começamos definindo o que são representações generalizadas. Antes, porém, vamos fazer uma observação quanto à notação:

Observação 5.1.1. Em geral, se $A$ é uma álgebra e $M$ é um espaço vetorial, uma ação de $A$ em $M$ que faz de $M$ um $A$-módulo é equivalente a um homomorfismo de álgebras $\phi: A \rightarrow \operatorname{End}_{k} M$. (Essa correspondência é dada pela relação $\phi(a)(m)=m$.a para todo $a \in A$ e $m \in M$ ). Sendo assim, entendendo essa correspondência como canônica, nos conceitos a serem descritos abaixo, um elemento $a$ de $A$ pode denotar tanto o elemento em si quanto sua imagem $\phi(a)$, que é o endomorfismo dado pela translação à direita por $a: m \mapsto m$. $a$ para todo $m \in M$. Isso é feito para não carregar a notação. 
Definição 5.1.2. • Uma $(\Gamma, \mathcal{A})$-representação generalizada é um par

$$
M=\left(\left(M_{i}\right)_{i \in \Gamma_{0}},\left(M_{\alpha}\right)_{\alpha \in \Gamma_{1}}\right)
$$

Para cada vértice $i \in \Gamma_{0}, M_{i}$ é um $A_{i}$-módulo, e para cada flecha $\alpha \in \Gamma_{1}, M_{\alpha}: M_{s(\alpha)} \rightarrow M_{e(\alpha)}$ é uma transformação $k$-linear. Além disso, $M$ é chamada de finitamente gerada se $M_{i}$ é finitamente gerada para cada vértice $i \in \Gamma_{0}$.

- Seja $\gamma=\sum_{i=1}^{t} \lambda_{t} \alpha_{i 1} \alpha_{i 2} \ldots \alpha_{i n_{1}}$ uma relação em $\Gamma$, onde $\lambda_{i} \in k$ e $\alpha_{i j} \in \Gamma_{1}$. Dizemos que uma representação generalizada $M$ tal como no item anterior satisfaz $\gamma$ se

$$
\sum_{i=1}^{t} \lambda_{t} M_{\alpha_{i n_{i}}} \circ \overline{\gamma_{i n_{i}}} \circ \ldots \circ M_{\alpha_{i 2}} \circ \overline{\gamma_{i 2}} \circ M_{\alpha_{i 1}}=0
$$

para toda escolha de caminhos $\gamma_{i j}$ sobre $\Sigma_{s\left(\alpha_{i j}\right)}$, com $1 \leq i \leq t, 2 \leq j \leq n_{i}$.

Dizemos ainda que $M$ satisfaz o conjunto de relações $I$ se satisfizer cada relação de $I$.

- Sejam $M=\left(\left(M_{i}\right)_{i \in \Gamma_{0}},\left(M_{\alpha}\right)_{\alpha \in \Gamma_{1}}\right)$ e $N=\left(\left(N_{i}\right)_{i \in \Gamma_{0}},\left(N_{\alpha}\right)_{\alpha \in \Gamma_{1}}\right)$ duas $(\Gamma, \mathcal{A})$-representações generalizadas. Daí um morfismo de representações generalizadas $f: M \rightarrow N$ é uma tupla $f=\left(f_{i}\right)_{i \in \Gamma_{0}}$, tal que, para cada $i \in \Gamma_{0}, f_{i}: M_{i} \rightarrow N_{i}$ é um morfismo de $A_{i}$-módulos; e tal que, para cada flecha $\alpha: i \rightarrow j \in \Gamma_{1}$, vale que $f_{j} M_{\alpha}=N_{\alpha} f_{i}$, ou seja, o seguinte diagrama comuta:

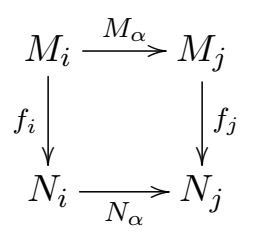

- A categoria das $(\Gamma, \mathcal{A})$-representações generalizadas que satisfazem $I$ será denotada por $\operatorname{Rep}_{k}(\Gamma, \mathcal{A}, I)$ (ou por $\operatorname{Rep}_{k}(\Gamma, \mathcal{A})$, se $I$ é vazio). A subcategoria plena das $(\Gamma, \mathcal{A}$ )-representações generalizadas finitamente geradas que satisfazem $I$ será denotada por $\operatorname{rep}_{k}(\Gamma, \mathcal{A}, I)$ (ou por $\operatorname{rep}_{k}(\Gamma, \mathcal{A})$, se $I$ é vazio $)$.

Apenas por brevidade, por vezes vamos nos referir às representações generalizadas simplesmente como representações, ainda que sejam conceitos diferentes.

O próximo passo será estabelecer a prometida equivalência entre $(\Gamma, \mathcal{A})$-representações que satisfazem $I$ e entre $\Lambda$-módulos, generalizando assim o Teorema 2.6.5 e o Teorema 2.4 de [6], onde a equivalência foi estabelecida apenas no caso $I=\emptyset$.

Teorema 5.1.3 (compare com [6],2.4). Existe um funtor

$$
F: \operatorname{Rep}_{k}(\Gamma, \mathcal{A}, I) \rightarrow \operatorname{Mod} k(\Gamma, \mathcal{A}, I)
$$

que é uma equivalênica $k$-linear entre a categoria das representações generalizadas sobre o par $(\Gamma, \mathcal{A})$ que satisfazem $I$ e entre a categoria $\operatorname{dos} k(\Gamma, \mathcal{A}, I)$-módulos.

Além disso, $F$ se restringe a uma equivalência 


$$
F: \operatorname{rep}_{k}(\Gamma, \mathcal{A}, I) \rightarrow \bmod k(\Gamma, \mathcal{A}, I)
$$

entre a categoria das representações generalizadas finitamente geradas sobre o par $(\Gamma, \mathcal{A})$ que satisfazem $I$ e entre a categoria $\operatorname{dos} k(\Gamma, \mathcal{A}, I)$-módulos finitamente gerados.

Demonstração. Vamos definir como $F$ age nos objetos, no caso, as representações.

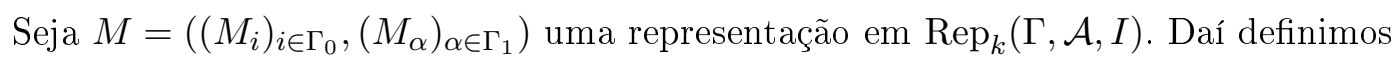

$$
F(M)=\bigoplus_{i \in \Gamma_{0}} M_{i}
$$

Resta definir a ação de $\Lambda$ sobre $F(M)$ de forma que $F(M)$ seja um objeto de $\operatorname{Mod} k(\Gamma, \mathcal{A}, I)$. Isso equivale a construir um homomorfismo de álgebras $\Phi: \Lambda \rightarrow$ End $F(M)$. A ideia é usar a Propriedade Universal das Álgebras Tensoriais (2.2.4). Sejam portanto $A_{\mathcal{A}}$ e $M_{\mathcal{A}}$ como na Observação 3.1.9.

Primeiro definimos a aplicação

$$
\phi_{0}: A_{\mathcal{A}} \rightarrow \operatorname{End}_{k} F(M)
$$

que é determinada por

$$
\forall i \in \Gamma_{0}, \forall a_{i} \in A_{i}, \forall\left(x_{l}\right)_{l \in \Gamma_{0}} \in F(M), \phi_{0}\left(a_{i}\right)\left(\left(x_{l}\right)_{l \in \Gamma_{0}}\right)=\left(\delta_{l i} x_{i} a_{i}\right)_{l \in \Gamma_{0}}
$$

onde $\delta_{l i}$ é um delta de Kronecker. Note que $\phi_{0}$ é um homomorfismo de álgebras.

Também definimos

$$
\phi_{1}: M_{\mathcal{A}} \rightarrow \operatorname{End}_{k} F(M)
$$

que é determinada pelo seguinte: para todo $\mathcal{A}$-caminho de comprimento $1 a_{i} \alpha a_{j}$, onde $\alpha: i \rightarrow j$ é uma flecha de $\Gamma$, para todos $a_{i} \in A_{i}, a_{j} \in A_{j}$, e toda tupla $\left(x_{l}\right)_{l \in \Gamma_{0}} \in F(M)$,

$$
\phi_{1}\left(a_{i} \alpha a_{j}\right)=\left(\delta_{l j} M_{\alpha}\left(x_{i} a_{i}\right) a_{j}\right)_{l \in \Gamma_{0}}
$$

Verifica-se que $\phi_{1}$ é um morfismo de $\left(A_{\mathcal{A}}-A_{\mathcal{A}}\right)$-bimódulos.

(Resumidamente, se $\imath_{l}: M_{l} \rightarrow F(M)$ e $\pi_{l}: F(M) \rightarrow M_{l}$ denotam, respectivamente, a inclusão canônica e a projeção canônica na $l$-ésima coordenada em relação à soma direta $F(M)=\oplus_{i \in \Gamma_{0}} M_{i}$, temos que $\phi_{0}\left(a_{i}\right)=\imath_{i} \circ a_{i} \circ \pi_{i}$ e $\left.\phi_{1}\left(a_{i} \alpha a_{j}\right)=\imath_{j} \circ a_{j} \circ M_{\alpha} \circ a_{i} \circ \pi_{i}\right)$.

Como $k(\Gamma, \mathcal{A})=T\left(A_{\mathcal{A}}, M_{\mathcal{A}}\right)$, pela Proposição 2.2.4, existe um homomorfismo de álgebras

$$
\phi: k(\Gamma, \mathcal{A}) \rightarrow \operatorname{End}_{k} F(M)
$$

unicamente determinado pela propriedade de que $\left.\phi\right|_{A_{\mathcal{A}}}=\phi_{0}$ e $\left.\phi\right|_{M_{\mathcal{A}}}=\phi_{1}$. Isso mostra que $F(M)$ é um $k(\Gamma, \mathcal{A})$-módulo. Para verificar que $F(M)$ é um módulo sobre $\Lambda=k(\Gamma, \mathcal{A}, I)$, é suficiente verificar que $\phi(I)=0$, pois disso segue pelo Teorema do Homomorfismo que $\phi$ induz um homomorfismo de álgebras $\Phi: k(\Gamma, \mathcal{A}) / I \rightarrow \operatorname{End}_{k} F(M)$.

Então vamos verificar que $\phi(I)=0$. Seja $\rho=\sum_{r=1}^{t} \lambda_{r} \alpha_{r 1} \ldots \alpha_{r n_{r}}$ uma relação de $I$, onde $\lambda_{r} \in k$ e as sequências $\alpha_{r 1} \ldots \alpha_{r n_{r}}$ são caminhos sobre $\Gamma$ que começam e terminam no mesmo vértice. Seja também, para cada $1 \leq r \leq t$ e $1 \leq j \leq n_{r}, \gamma_{r j}$ um caminho sobre $\Sigma_{s\left(\alpha_{r j}\right)}$. Daí: 


$$
\begin{aligned}
& \phi\left(\sum_{r=1}^{t} \lambda_{r} \alpha_{r 1} \overline{\gamma_{r 2}} \alpha_{r 2} \ldots \overline{\gamma_{r n_{r}}} \alpha_{r n_{r}}\right) \\
& \quad=\sum_{r=1}^{t} \lambda_{r} \phi\left(\alpha_{r 1} \overline{\gamma_{r 2}} \alpha_{r 2} \ldots \overline{\gamma_{r n_{r}}} \alpha_{r n_{r}}\right) \\
& =\sum_{r=1}^{t} \lambda_{r} \imath_{e\left(\alpha_{r n_{r}}\right)} \circ M_{\alpha_{r n_{r}}} \circ \overline{\gamma_{r n_{r}}} \circ \ldots M_{\alpha_{r 2}} \circ \overline{\gamma_{r 2}} \circ M_{\alpha_{r 1}} \circ \pi_{s\left(\alpha_{r 1}\right)} \\
& =\imath_{e\left(\alpha_{1 n_{1}}\right)} \circ\left(\sum_{r=1}^{t} \lambda_{r} M_{\alpha_{r n_{r}}} \circ \overline{\gamma_{r n_{r}}} \circ \ldots M_{\alpha_{r 2}} \circ \overline{\gamma_{r 2}} \circ M_{\alpha_{r 1}}\right) \circ \pi_{s\left(\alpha_{11}\right)} \\
& \quad=0
\end{aligned}
$$

A última igualdade vale porque $M$ satisfaz $\rho$. Isso conclui a exposição de como $F$ age nos objetos. Vejamos como age nos morfismos.

Seja $f=\left(f_{i}\right)_{i \in \Gamma_{0}}: M \rightarrow N$ um morfismo de representações, onde $M=\left(\left(M_{i}\right)_{i \in \Gamma_{0}},\left(M_{\alpha}\right)_{\alpha \in \Gamma_{1}}\right)$ e $N=\left(\left(N_{i}\right)_{i \in \Gamma_{0}},\left(N_{\alpha}\right)_{\alpha \in \Gamma_{1}}\right)$ são representações que satisfazem $I$. Então cada $f_{i}: M_{i} \rightarrow N_{i}$ é um morfismo de $A_{i}$-módulos, e portanto podemos definir uma aplicação linear

$$
F(f)=F(M)=\bigoplus_{i \in \Gamma_{0}} M_{i} \rightarrow F(N)=\bigoplus_{j \in \Gamma_{0}} N_{i}
$$

ao estabelecer que a coordenada $(i, j)$ de $F(f)$ é $\delta_{i j} f_{i}$. Verifica-se que $F(f)$ é um morfismo de $\Lambda$-módulos e que $F$ assim definido é um funtor.

Agora definimos aquele que vai ser o funtor quase-inverso de $F$ :

$$
G: \operatorname{Mod} k(\Gamma, \mathcal{A}) \rightarrow \operatorname{Rep}_{k}(\Gamma, \mathcal{A})
$$

Seja $M$ um $\Lambda$-módulo. Precisamos definir uma $(\Gamma, \mathcal{A})$-representação $G(M)=\left(\left(M_{i}\right)_{i \in \Gamma_{0}},\left(\phi_{\alpha}\right)_{\alpha \in \Gamma_{1}}\right)$ que satisfaz $I$.

- Para cada $i \in \Gamma_{0}, M_{i}$ é definido por $M_{i} \doteq M .1_{i}$. Note que este é um $A_{i}$-módulo, pois $M_{i} \cdot A_{i}=\left(M \cdot 1_{i}\right) \cdot A_{i}=M \cdot A_{i}=M \cdot\left(A_{i} \cdot 1_{i}\right)=\left(M \cdot A_{i}\right) \cdot 1_{i} \subseteq M \cdot 1_{i}=M_{i}$.

- Se $\alpha: i \rightarrow j \in \Gamma_{1}$ é uma flecha, então definimos uma aplicação $\phi_{\alpha}: M_{i} \rightarrow M_{j}$ por $\phi_{\alpha}(m)=$ $m . \alpha$. Note que ela está bem definida, pois se $m \in M_{i}, m . \alpha=m \cdot\left(\alpha \cdot 1_{j}\right)=(m . \alpha) \cdot 1_{j} \in M \cdot 1_{j}=$ $M_{j}$. E de fato $M_{\alpha}$ é $k$-linear, pois se $\lambda \in k$ e $m \in M, M_{\alpha}\left(\lambda m .1_{i}\right)=\left(\lambda m .1_{i}\right) \alpha=\lambda\left(\left(m .1_{i}\right) \alpha\right)=$ $\lambda M_{\alpha}\left(m \cdot 1_{i}\right)$.

Resta ver que $G(M)$ assim definida satisfaz $I$. Seja novamente $\rho=\sum_{r=1}^{t} \lambda_{r} \alpha_{r 1} \ldots \alpha_{r n_{r}}$ uma relação de $I$, onde $\lambda_{r} \in k$ e as sequências $\alpha_{r 1} \ldots \alpha_{r n_{r}}$ são caminhos sobre $\Gamma$ que começam e terminam no mesmo vértice. Seja também, para cada $1 \leq r \leq t$ e $1 \leq j \leq n_{r}, \gamma_{r j}$ um caminho sobre $\Sigma_{s\left(\alpha_{r j}\right)}$. Daí, para $m \in M_{s\left(\alpha_{r 1}\right)}$, 


$$
\begin{aligned}
& \left(\sum_{r=1}^{t} \lambda_{r} M_{\alpha_{r n_{r}}} \circ \overline{\gamma_{r n_{r}}} \circ \ldots M_{\alpha_{r 2}} \circ \overline{\gamma_{\alpha_{r 2}}} \circ M_{\alpha_{r 1}}\right)(m) \\
& =\left(\sum_{r=1}^{t} \lambda_{r} M_{\alpha_{r n_{r}}} \circ \overline{\gamma_{r n_{r}}} \circ \ldots M_{\alpha_{r 2}} \circ \overline{\gamma_{r 2}}\right)\left(m \alpha_{r 1}\right) \\
& =\left(\sum_{r=1}^{t} \lambda_{r} M_{\alpha_{r n_{r}}} \circ \overline{\gamma_{r n_{r}}} \circ \ldots M_{\alpha_{r 2}}\right)\left(m \alpha_{r 1} \overline{\gamma_{r 2}}\right) \\
& =\ldots=\sum_{r=1}^{t} \lambda_{r} m \alpha_{r 1} \overline{\gamma_{r 2}} \ldots \overline{\gamma_{r n_{r}}} \alpha_{r n_{r}} \\
& =m\left(\sum_{r=1}^{t} \lambda_{r} \alpha_{r 1} \overline{\gamma_{r 2}} \ldots \overline{\gamma_{r n_{r}}} \alpha_{r n_{r}}\right) \\
& =0
\end{aligned}
$$

A última igualdade vale porque a expressão que multiplica $m$ é igual a $0 \mathrm{em} \Lambda$. Provamos portanto que $G(M)$ é um objeto em $\operatorname{Rep}_{k}(\Gamma, \mathcal{A}, I)$.

Seja $g: M \rightarrow N$ um morfismo em $\operatorname{Mod} \Lambda$. Então definimos sua imagem por $G$ :

$$
\begin{aligned}
& G(g)=\left(G(g)_{i}\right)_{i \in \Gamma_{0}} \\
& G(g)_{i}: M_{i} \rightarrow N_{i},\left.G(g)_{i} \doteq g\right|_{M_{i}}
\end{aligned}
$$

É imediato constatar que $G(g)_{i}$ está bem definido e é um morfismo de $A_{i}$-módulos para cada $i \in \Gamma_{0}$. Vamos verificar que $G(g)$ é um morfismo de representações. Seja $\alpha: i \rightarrow j$ uma flecha em $\Gamma$. Então, para todo $m \in M, G(g)_{j} \circ M_{\alpha}\left(m .1_{i}\right)=G(g)_{j}(m \alpha)=g(m \alpha)=g(m) \alpha=G(g)_{i}\left(m .1_{i}\right) \alpha=$ $N_{\alpha} \circ G(g)_{i}\left(m .1_{i}\right)$. Portanto $G(g)_{j} \circ M_{\alpha}=N_{\alpha} \circ G(g)_{i}$, ou seja, o seguinte diagrama comuta:

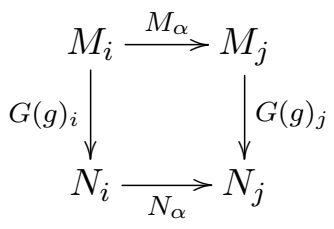

Logo $G(g)$ é um morfismo de representações. É direto verificar que $G$ definido dessa forma é funtor.

Também verifica-se diretamente que:

- F e $G$ são quase-inversos e portanto são equivalências.

- F leva representações finitamente geradas em módulos finitamente gerados, enquanto que $G$ faz o contrário. Logo as restrições destes funtores a essas subcategorias continuam sendo equivalências quase-inversas.

Tendo como ferramenta a equivalência entre categorias discutida acima, temos condições de estudar, nas próximas seções, as representações associadas aos módulos simples, projetivos e injetivos 
sobre uma ACGR, generalizando assim a bem conhecida descrição que é feita no caso de álgebra de caminhos ordinárias.

\subsection{Módulos simples}

Nesta seção apresentamos uma proposição que descreve completamente como são as representações associadas aos $k(\Gamma, \mathcal{A}, I)$-módulos simples.

Proposição 5.2.1. Seja $i \in \Gamma_{0}$ e seja $S$ um $A_{i}$-módulo simples. Então a representação generalizada dada por

$$
\begin{gathered}
\bar{S}=\left(\left(M_{j}\right)_{j \in \Gamma_{0}},\left(\phi_{\alpha}\right)_{\alpha \in \Gamma_{1}}\right), \text { com } \\
M_{j}= \begin{cases}S & \text { se } j=i \\
0 & \text { se } j \neq i\end{cases} \\
\phi_{\alpha}=0 \forall \alpha \in \Gamma_{1}
\end{gathered}
$$

é simples. Além disso, toda representação generalizada simples sobre $(\Gamma, \mathcal{A}, I)$ é isomorfa a uma tendo a forma acima.

Demonstração. Note que $\bar{S}$ satisfaz $I$, porque todas as transformações lineares que integram $\bar{S}$ são nulas.

Agora verificamos que a representação dada acima é simples. Seja $N=\left(\left(N_{j}\right)_{j \in \Gamma_{0}},\left(\psi_{\alpha}\right)_{\alpha \in \Gamma_{1}}\right)$ uma subrepresentação de $\bar{S}$. Então, para cada $j \in \Gamma_{0}, N_{j}$ é isomorfo a um $A_{j}$-submódulo de $M_{j}$. Isso implica que $N_{j}=0$ para $j \neq i$. Além disso, como $M_{i}=S$ é simples sobre $A_{i}$, devemos ter $N_{i}=0$ ou $N_{i}=S$. No primeiro caso, $N=0$, e no segundo, $N=\bar{S}$. Isso prova que $\bar{S}$ é simples.

Note que temos, em cada vértice $i$, um total de $\operatorname{rk} K_{0}\left(A_{i}\right)$ representações tendo a forma do enunciado, graças aos Teoremas 2.2.12 e 2.2.19. Logo já temos $\sum_{i \in \Gamma_{0}} \mathrm{rk} K_{0}\left(A_{i}\right)$ representações simples, e elas não são isomorfas entre si. Como, pela Observação 3.1.7,

$$
\operatorname{rk} K_{0}(k(\Gamma, \mathcal{A}))=\sum_{i \in \Gamma_{0}} \operatorname{rk} K_{0}\left(A_{i}\right)
$$

concluímos que essas representações formam um conjunto completo de representações simples sobre $(\Gamma, \mathcal{A}, I)$.

\subsection{Realizando um $A_{i}$-módulo como um $\Lambda$-módulo}

Seja $i \in \Gamma_{0}$, e seja $M_{A_{i}}$ um $A_{i}$-módulo (à direita). Nós vamos estudar nesta Seção duas maneiras de enxergar $M$ como um $\Lambda$-módulo (haverá uma terceira depois). A primeira é bastante natural,e a segunda consiste essencialmente na conhecida técnica de extensão de escalares. É interessante dedicar notações distintas para cada maneira.

No primeiro caso, considere a $(\Gamma, \mathcal{A}, I)$-representação generalizada abaixo: 


$$
\begin{gathered}
I(M)=\left(\left(M_{j}\right)_{j \in \Gamma_{0}},\left(\phi_{\alpha}\right)_{\alpha \in \Gamma_{1}}\right), \mathrm{com} \\
M_{j}= \begin{cases}M & \text { se } j=i \\
0 & \text { se } j \neq i\end{cases} \\
\phi_{\alpha}=0 \forall \alpha \in \Gamma_{1}
\end{gathered}
$$

Então, pela equivalência de que trata o Teorema 5.1.3, esta representação nos fornece um $\Lambda$ módulo. Como $I(M)$ e $M$ têm o mesmo espaço vetorial subjacente, podemos, por um certo abuso de notação, denotar $I(M)=M$.

Na verdade, para cada vértice $i$ temos um funtor $I_{i}: \bmod A_{i} \rightarrow \bmod \Lambda$ que vamos chamar de funtor inclusão. (Podemos ainda denotá-lo apenas por $I$ se estiver claro de qual vértice se trata). Nós já definimos a imagem dos objetos acima, e a imagem de morfismo é definida de forma óbvia. Também é fácil ver que $I$ de fato merece ser chamado de funtor inclusão, porque ele é covariante, fiel e pleno.

Daqui para frente, se não houver menção em contrário, estaremos assumindo que estamos vendo $M$ como um $\Lambda$-módulo desta forma. Se lembrarmos a Seção 5.2, notamos que para obter os $\Lambda$ módulos simples, tudo o que fizemos foi realizar os $A_{j}$-módulos simples como $\Lambda$-módulos para todo $j$.

Vamos introduzir a outra forma. Denote $A_{\mathcal{A}}=\prod_{j \in \Gamma_{0}} A_{j}$. Então para cada $i \in \Gamma_{0}$ temos um epimorfismo de álgebras canônico $\pi_{i}: A_{\mathcal{A}}=\prod_{j \in \Gamma_{0}} A_{j} \rightarrow A_{i}$. Se $M$ é um $A_{i}$-módulo, por restrição de escalares via $\pi_{i}$, ele é também um $A_{\mathcal{A}}$-módulo. Ou seja, se $m \in M$ e $\left(a_{j}\right)_{j \in \Gamma_{0}} \in A_{\mathcal{A}}$, temos que $m .\left(a_{j}\right)_{j}=m \cdot a_{i}$.

Agora lembrando a definição de ACG's (mais especificamente, a Observação 3.1.9), temos que $k(\Gamma, \mathcal{A})$ é uma $A_{\mathcal{A}^{-}}$álgebra tensorial sobre um certo $\left(A_{\mathcal{A}}-A_{\mathcal{A}}\right)$-bimódulo aqui denotado por $M_{\mathcal{A}}$, ou seja, $k(\Gamma, \mathcal{A})=T\left(A_{\mathcal{A}}, M_{\mathcal{A}}\right)$. E mais, como $\Lambda$ é o quociente $k(\Gamma, \mathcal{A}) / I, \Lambda$ é também um $\left(A_{\mathcal{A}}-A_{\mathcal{A}}\right)$ bimódulo que contém $A_{\mathcal{A}}$ como subálgebra. Portanto faz sentido considerar a extensão de escalares de $M$ a $\Lambda$. Vamos denotá-la por $\mathscr{C}_{i}(M)=M \otimes_{A_{\mathcal{A}}} \Lambda$. Apenas enfatizando, como $\Lambda$ é um $\Lambda$-módulo à direita, também $\mathscr{C}_{i}(M)$ é um $\Lambda$-módulo à direita. Esta é uma outra maneira de realizar $M$ como um $\Lambda$-módulo, contudo, diferentemente da anterior, o espaço vetorial subjacente pode ser diferente do de $M$.

Definição 5.3.1. $\mathscr{C}_{i}(M)$ é chamado de cone sobre $M$.

A razão pela qual damos o nome de cone é o formato que a representação associada a $\mathscr{C}_{i}(M)$ tem, conforme vai ficar mais aparente com a descrição que faremos.

Proposição 5.3.2. Se $M$ e $N$ são ambos $A_{i}$-módulos, então $\mathscr{C}_{i}(M \oplus N) \cong \mathscr{C}_{i}(M) \oplus \mathscr{C}_{i}(N)$ como $\Lambda$-módulos.

Demonstração. $\mathscr{C}_{i}(M \oplus N)=(M \oplus N) \otimes_{A_{\mathcal{A}}} \Lambda \cong\left(M \otimes_{A_{\mathcal{A}}} \Lambda\right) \oplus\left(N \otimes_{A_{\mathcal{A}}} \Lambda\right)=\mathscr{C}_{i}(M) \oplus \mathscr{C}_{i}(N)$.

Proposição 5.3.3. Se $P$ é um $A_{i}$-módulo projetivo, então $\mathscr{C}_{i}(P)$ é um $\Lambda$-módulo projetivo.

Demonstração. Seja $g: M \rightarrow N$ um epimorfismo entre $\Lambda$-módulos. Como $\Lambda$ é um $\Lambda$-módulo projetivo, 


$$
\operatorname{Hom}_{\Lambda}(\Lambda, g): \operatorname{Hom}_{\Lambda}(\Lambda, M) \rightarrow \operatorname{Hom}_{\Lambda}(\Lambda, N)
$$

é um epimorfismo. Como $A_{i}=1_{i} A_{\mathcal{A}}$ e $1_{i}$ é um elemento idempotente de $A_{\mathcal{A}}, A_{i}$ é um $A_{\mathcal{A}^{-}}$ módulo projetivo. Pela hipótese, $P$ é um somando direto de algum $A_{i}$-módulo da forma $A_{i}^{m}, m \in \mathbb{N}$, e portanto também $P$ é projetivo como $A_{\mathcal{A}}$-módulo. Segue que

$$
\operatorname{Hom}_{A_{\mathcal{A}}}\left(P, \operatorname{Hom}_{\Lambda}(\Lambda, g)\right): \operatorname{Hom}_{A_{\mathcal{A}}}\left(P, \operatorname{Hom}_{\Lambda}(\Lambda, M)\right) \rightarrow \operatorname{Hom}_{A_{\mathcal{A}}}\left(P, \operatorname{Hom}_{\Lambda}(\Lambda, N)\right)
$$

é um epimorfismo. Finalmente, pelo Teorema da Adjunção,

$$
\operatorname{Hom}_{\Lambda}\left(P \otimes_{A_{\mathcal{A}}} \Lambda, g\right): \operatorname{Hom}_{\Lambda}\left(P \otimes_{A_{\mathcal{A}}} \Lambda, M\right) \rightarrow \operatorname{Hom}_{\Lambda}\left(P \otimes_{A_{\mathcal{A}}} \Lambda, N\right)
$$

é um epimorfismo. Isso prova que $P \otimes_{A_{\mathcal{A}}} \Lambda$ é um $\Lambda$-módulo projetivo.

Observação 5.3.4. É útil observar que

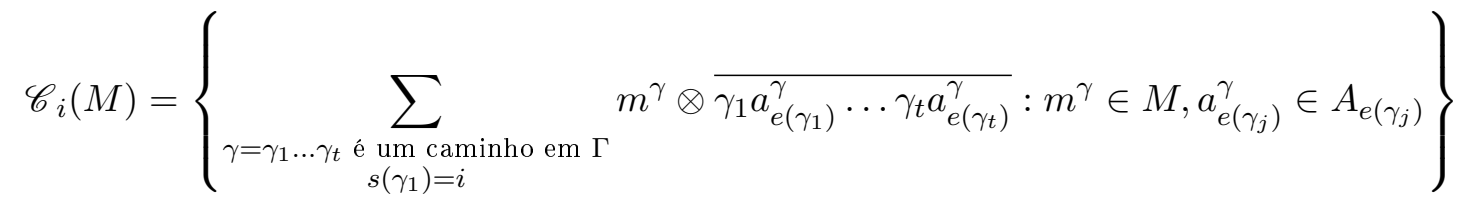

Essa igualdade segue da constatação de que $\mathscr{C}_{i}(M)=M \otimes_{A_{\mathcal{A}}} \Lambda=M .1_{i} \otimes_{A_{\mathcal{A}}} \Lambda=M \otimes_{A_{\mathcal{A}}} 1_{i} . \Lambda$.

O objetivo final desta seção é descrever as representações generalizadas associadas a um cone.

Proposição 5.3.5. Suponha que $\Gamma$ é acíclica. Seja $M$ um $A_{i}$-módulo. Pelo Teorema 5.1.3 acima, $\mathscr{C}_{i}(M)$ corresponde a uma representação que denotaremos por $\left(\left(M_{j}\right)_{j \in \Gamma_{0}},\left(\phi_{\alpha}\right)_{\alpha \in \Gamma_{1}}\right)$.

Seja $\left\{a_{1}^{l}, \ldots, a_{\operatorname{dim}_{\mathrm{k}} A_{l}}^{l}\right\}$ uma base de $A_{l}$ sobre $k$ para cada vértice $l \in \Gamma_{0}$, e seja $\left\{m_{1}, \ldots, m_{\operatorname{dim}_{\mathrm{k}} M}\right\}$ uma base de $M$ sobre $k$.

Com essas notações, vale que $M_{i}=M$ e que, se $j \in \Gamma_{0}$ é distinto de $i$, então $M_{j}$ é isomorfo ao $A_{j}$-módulo livre tendo como base as classes de equivalência das sequências formais da forma

$$
m_{p} \gamma_{1} a_{i_{2}}^{s\left(\gamma_{2}\right)} \ldots a_{i_{r}}^{s\left(\gamma_{r}\right)} \gamma_{r}
$$

onde $\gamma_{1} \ldots \gamma_{r}$ é um caminho que vai de $i$ a $j, 1 \leq p \leq \operatorname{dim}_{\mathrm{k}} M$ e $1 \leq i_{l} \leq \operatorname{dim}_{\mathrm{k}} A_{s\left(\gamma_{l}\right)}$ para todo $l<r$.

Além disso, se $\alpha: j \rightarrow j^{\prime}$ é uma flecha, então $\phi_{\alpha}$ é única transformação linear que satisfaz $\phi_{\alpha}\left(\overline{m_{p} \gamma_{1} a_{i_{2}}^{s\left(\gamma_{2}\right)} \ldots a_{i_{r}}^{s\left(\gamma_{r}\right)} \gamma_{r}} a_{i_{r+1}}^{j}\right)=\overline{m_{p} \gamma_{1} a_{i_{2}}^{s\left(\gamma_{2}\right)} \ldots a_{i_{r}}^{s\left(\gamma_{r}\right)} \gamma_{r} a_{i_{r+1}}^{j} \alpha .}$

Observação 5.3.6. Se $I=0$, então é mais fácil ver como fica a representação de $\mathscr{C}_{i}(M)$ : vale que $M_{i}=M$ e se $j \neq i, M_{j} \cong A_{j}^{n_{j}}$, onde

$$
n_{j}=\sum_{\gamma: i=i_{0} \rightarrow i_{1} \rightarrow \ldots \rightarrow i_{r+1}=j \text { é caminho } i \rightsquigarrow j}\left(\operatorname{dim}_{\mathrm{k}} M\right) \cdot\left(\operatorname{dim}_{\mathrm{k}} A_{i_{1}}\right) \ldots .\left(\operatorname{dim}_{\mathrm{k}} A_{i_{r}}\right)
$$

Em particular, se não existe caminho de $i$ para $j, M_{j}=0$. 
Demonstração. Para entender a demonstração, é necessário lembrar-se da equivalência $G$ descrita na demonstração do Teorema 5.1.3.

Pela Observação 5.3.4 acima, e pelo fato de $\Gamma$ ser acíclica,

$$
M_{i}=\mathscr{C}_{i}(M) \cdot 1_{i} \cong\left\{\sum_{\gamma: i \rightsquigarrow i} m^{\gamma}: m^{\gamma} \in M\right\}=\{m: m \in M\}=M
$$

Para $j \neq i$, temos que

$$
\begin{gathered}
M_{j}=\mathscr{C}_{i}(M) \cdot 1_{j}=\left\{\sum_{\gamma=\gamma_{1} \ldots \gamma_{r-1}: i \rightsquigarrow j} m^{\gamma} \otimes \overline{\gamma_{1} a_{2}^{\gamma} \gamma_{2} \ldots a_{r}^{\gamma} \gamma_{r} a_{r+1}^{\gamma}}:\right. \\
\left.m^{\gamma} \in M, a_{l}^{\gamma} \in A_{s\left(\gamma_{l}\right)} \forall l \leq r, \text { e } a_{r+1}^{\gamma} \in A_{j}\right\}
\end{gathered}
$$

Como $\left\{a_{1}^{l}, \ldots, a_{\operatorname{dim}_{\mathrm{k}} A_{l}}^{l}\right\}$ é uma base de $A_{l}$ sobre $k$ para cada $l$, e como $\left\{m_{1}, \ldots, m_{\operatorname{dim}_{\mathrm{k}} M}\right\}$ é uma base de $M$ sobre $k$, a expressão acima é igual a

$$
\begin{gathered}
\operatorname{span}_{k}\left\{m_{p} \otimes \overline{\gamma_{1} a_{i_{2}}^{s\left(\gamma_{2}\right)} \ldots a_{i_{r}}^{s\left(\gamma_{r}\right)} \gamma_{r}} a_{r+1}: \gamma_{1} \ldots \gamma_{r} \text { é um caminho } i \rightsquigarrow j,\right. \\
\left.1 \leq p \leq \operatorname{dim}_{\mathrm{k}} M, 1 \leq i_{l} \leq \operatorname{dim}_{\mathrm{k}} A_{s\left(\gamma_{l}\right)} \forall l \leq r, \text { e } a_{r+1} \in A_{j}\right\}
\end{gathered}
$$

Para facilitar a notação, denote $\left\{\theta_{1}, \ldots, \theta_{n_{j}}\right\}=\left\{m_{p} \otimes \overline{\gamma_{1} a_{i_{2}}^{s\left(\gamma_{2}\right)} \ldots a_{i_{r}}^{s\left(\gamma_{r}\right)} \gamma_{r}}\right\}$. Então a expressão 5.1 é igual a

$$
\operatorname{span}_{k}\left\{\theta_{l} a: 1 \leq l \leq n_{j}, a \in A_{j}\right\}
$$

e é direto verificar que este, por sua vez, é isomorfo ao $A_{j}$-módulo livre tendo como base $\theta_{1}, \ldots, \theta_{n_{j}}$, que é o que queríamos provar.

Seja $\alpha: j \rightarrow j^{\prime}$ uma flecha de $\Gamma_{1}$. De novo, pelo Teorema 5.1.3, $\phi_{\alpha}$ é dada por

$$
\begin{aligned}
\phi_{\alpha}: & M_{l} \rightarrow M_{j} \\
& \mathscr{C}_{i}(M) 1_{l} \rightarrow \mathscr{C}_{i}(M) 1_{j} \\
& m 1_{l} \mapsto m \alpha
\end{aligned}
$$

com $m \in \mathscr{C}_{i}(M)$. Portanto $\phi_{\alpha}$ tem a forma descrita no enunciado, concluindo a demonstração.

\subsection{Módulos projetivos}

Nesta seção aplicamos as ideias introduzidas na Seção 5.3 para descrever os $\Lambda$-módulos projetivos indecomponíveis. Isso já havia sido feito para as ACGs em [12], porém aqui vamos aprofundar essa discussão, incluindo o contexto mais geral tratado aqui, que é o das ACGRs.

Se lembrarmos a Seção 5.2, sabemos que os $\Lambda$-módulos simples foram obtidos vendo os $A_{j}$ módulos simples como $\Lambda$-módulos para cada $j \in \Gamma_{0}$. Este também será o caso para os projetivos, 
exceto que em vez de ver os $A_{j}$-módulos projetivos como $\Lambda$-módulos do jeito mais natural, vamos usar o conceito de cone.

Aqui o Teorema 2.2.12 será fundamental. Vamos recuperar a notação já usada antes: para cada $i \in \Gamma_{0}$, seja $E_{i}=\left\{e_{i 1}, \ldots, e_{i s_{i}}\right\}$ um conjunto completo de idempotentes primitivos dois a dois ortogonais em $A_{i}$. Então todo $A_{i}$-módulo projetivo indecomponível é isomorfo a $P_{i}^{j} \doteq e_{i j} A_{i}$ para algum $1 \leq j \leq s_{i}$. Além disso, $E=\left\{\overline{e_{i j}}: i \in \Gamma_{0}, 1 \leq j \leq s_{i}\right\}$ é um conjunto completo de idempotentes primitivos dois a dois ortogonais em $\Lambda$. Portanto todo $\Lambda$-módulo projetivo indecomponível é isomorfo a $P(i, j) \doteq e_{i j} \Lambda$ para algum par de índices $i \in \Gamma_{0}$ e $1 \leq j \leq s_{i}$.

Proposição 5.4.1. Para cada $i \in \Gamma_{0}$ e $1 \leq j \leq s_{i}, P(i, j)=\mathscr{C}_{i}\left(P_{i}^{j}\right)$.

Demonstração. Lembrando a Observação 5.3.4, temos que

$$
\begin{aligned}
& \mathscr{C}_{i}\left(P_{i}^{j}\right)=\left\{\sum_{\substack{\gamma=\gamma_{1} \ldots \gamma_{t} \\
\text { é um caminho em } \Gamma \\
s\left(\gamma_{1}\right)=i}} m^{\gamma} \otimes \overline{\gamma_{1} a_{e\left(\gamma_{1}\right)}^{\gamma} \cdots \gamma_{t} a_{e\left(\gamma_{t}\right)}^{\gamma}}: m^{\gamma} \in P_{i}^{j}, a_{e\left(\gamma_{j}\right)}^{\gamma} \in A_{e\left(\gamma_{j}\right)}\right\}
\end{aligned}
$$

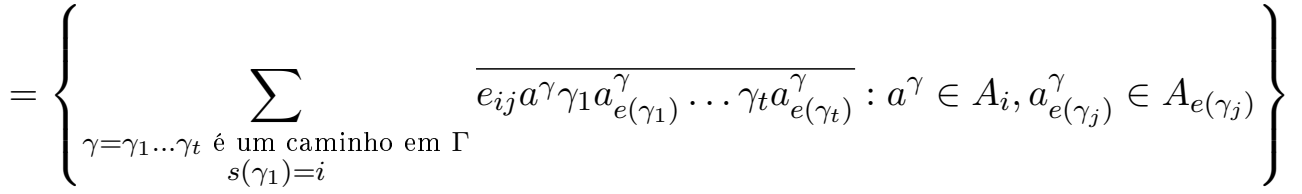

$$
\begin{aligned}
& =\overline{e_{i j}} \Lambda=P(i, j)
\end{aligned}
$$

Graças à última proposição e à Proposição 5.3.5, já conseguimos calcular as representações associadas a módulos projetivos indecomponíveis.

Nos exemplos práticos, no entanto, podem surgir problemas porque as matrizes das transformações $k$-lineares $\phi_{\alpha}$ podem ser muito grandes, e, dada sua dependência na escolha da $k$-base das álgebras $A_{i}$ ou de $P_{i}^{j}$, pode haver confusão. Para evitar isso, é conveniente lançar mão de matrizes em blocos. Vamos dar mais detalhes disso na observação abaixo e nos exemplos.

Observação 5.4.2. Seja $V$ um espaço vetorial com dimensão 1 e base fixada $\{v\}$ sobre $k$ e seja $A$ uma $k$-álgebra. Daí existe uma aplicação linear que será tratada como canônica daqui para frente: ela é definida por $\mu: V \rightarrow A, \mu(\lambda . v)=\lambda .1_{A}$, onde $\lambda \in k$.

Embora o espaço vetorial $V$ possa variar, a letra $\mu$ será sempre usada para essa aplicação.

Exemplo 5.4.3. Seja $A$ a álgebra de caminhos dada pela aljava

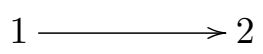

Então há dois $A$-módulos projetivos indecomponíveis, a saber,

$$
P_{1}: k \stackrel{i d}{\longrightarrow} k \quad P_{2}: 0 \longrightarrow k
$$

Seja agora $\Lambda$ a ACG dada por

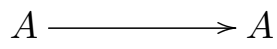


De acordo com as discussões acima, existem $4 \Lambda$-módulos projetivos indecomponíveis, que são:

$$
\begin{array}{ll}
P(1,1): P_{1} \stackrel{\left[\begin{array}{ll}
\mu & 0 \\
0 & \mu
\end{array}\right]}{\longrightarrow} A^{2} & P(1,2): P_{2} \stackrel{[\mu]}{\longrightarrow} A \\
P(2,1): 0 \longrightarrow P_{1} & P(2,2): 0 \stackrel{ }{\longrightarrow} P_{2}
\end{array}
$$

Estamos também em condições de descrever as representações associadas aos radicais dos módulos projetvos, conforme expresso na proposição abaixo.

Proposição 5.4.4. Com as mesmas notações de antes, seja $i \in \Gamma_{0}$ e $1 \leq j \leq s_{i}$. Denote $P(i, j)=\left(\left(M_{l}\right)_{l \in \Gamma_{0}},\left(\phi_{\alpha}\right)_{\alpha \in \Gamma_{1}}\right)$. Então o radical de $P(i, j)$ é dado pela representação $\operatorname{rad} P(i, j)=$ $\left(\left(N_{l}\right)_{l \in \Gamma_{0}},\left(\psi_{\alpha}\right)_{\alpha \in \Gamma_{1}}\right)$, onde $N_{i}=\operatorname{rad} P_{i}^{j}$, para cada $l \in \Gamma_{0} \operatorname{com} l \neq i, N_{l}=M_{l}$, e para cada $\alpha \in \Gamma_{1}$, $\psi_{\alpha}=\left.\phi_{\alpha}\right|_{M_{s(\alpha)}}$.

Demonstração. Seja $N=\left(\left(N_{l}\right)_{l \in \Gamma_{0}},\left(\psi_{\alpha}\right)_{\alpha \in \Gamma_{1}}\right)$. Note que $N$ satisfaz $I$ porque $M$ satisfaz. Queremos provar que $N=\operatorname{rad} P(i, j)$. Note que, se $l \neq i, N_{l}=M_{l}$, então $M_{l} / N_{l}=0$. Além disso, $M_{i}=P_{i}^{j}$ e $N_{i}=\operatorname{rad} P_{i}^{j}, \operatorname{logo} M_{i} / N_{i}=P_{i}^{j} / \operatorname{rad} P_{i}^{j}$. Isso implica que $P(i, j) / N$ é isomorfo ao $A_{i}$-módulo $P_{i}^{j} / \operatorname{rad} P_{i}^{j}$ realizado como $\Lambda$-módulo. Como $P_{i}^{j}$ é um $A_{i}$-módulo projetivo indecomponível, $P_{i}^{j} / \operatorname{rad} P_{i}^{j}$ é um $A_{i}$-módulo simples, e é também um $\Lambda$-módulo simples quando visto como $\Lambda$-módulo, vide Seção 5.2. Isso significa que $P(i, j) / N$ é um $\Lambda$-módulo simples. Provamos que $N$ é um ideal maximal de $P(i, j)$, e como $P(i, j)$ é projetivo indecomponível, ele tem um único ideal maximal, que é $\operatorname{rad} P(i, j)$. Isso conclui a demonstração de que $N=\operatorname{rad} P(i, j)$.

Exemplo 5.4.5. Continuamos o Exemplo 5.4.3 para aplicar a Proposição 5.4.4 e assim obter o radical dos 4 projetivos indecomponíveis. Temos portanto:

$$
\operatorname{rad} P(1,1): \operatorname{rad} P_{1} \stackrel{\left[\begin{array}{l}
0 \\
\mu
\end{array}\right]}{\longrightarrow} A^{2}
$$

$\operatorname{rad} P(2,1): 0 \longrightarrow \operatorname{rad} P_{1}$ $\operatorname{rad} P(1,2): 0 \longrightarrow A$

$\operatorname{rad} P(2,2): 0 \longrightarrow 0$

\section{5 Álgebra Oposta e Dualidade}

O objetivo desta seção é obter alguns lemas úteis relacionando álgebras opostas, aljavas opostas e o funtor dualidade. Lembramos que estes conceitos já foram lembrados no Capítulo 2.

Proposição 5.5.1. Seja $\Lambda=k(\Gamma, \mathcal{A})$ uma $\mathrm{ACG}$, onde $\Gamma$ é uma aljava e $\mathcal{A}=\left\{A_{i}: i \in \Gamma_{0}\right\}$ é uma família de álgebras. Denote por $\Gamma^{o p}$ a aljava oposta de $\Gamma$ e denote $\mathcal{A}^{o p}=\left\{A_{i}^{o p}: i \in \Gamma_{0}\right\}$. Então $\Lambda^{o p} \cong k\left(\Gamma^{o p}, \mathcal{A}^{o p}\right)$. Além disso, se $\Gamma$ tem um conjunto de relações $I$, então, se $I^{o p}$ é o conjunto de relações em $\Gamma^{o p}$ obtido invertendo-se as flechas das relações em $I$, vale o isomorfismo $(\Lambda / I)^{o p} \cong k\left(\Gamma^{o p}, \mathcal{A}^{o p}\right) / I^{o p}$, ou seja, $(k(\Gamma, \mathcal{A}, I))^{o p} \cong k\left(\Gamma^{o p}, \mathcal{A}^{o p}, I^{o p}\right)$.

Demonstração. Lembramos que na definição de ACG's nós definimos $k(\Gamma, \mathcal{A})$ como um quociente do espaço vetorial denotado por $k[\Gamma, \mathcal{A}]$. Vamos então usar a notação auxiliar $k(\Gamma, \mathcal{A}) \doteq k[\Gamma, \mathcal{A}] / \sim$. 
Por questão de clareza, vamos também denotar a classe de equivalência (em relação a $\sim$ ) de um $\mathcal{A}$-caminho $x$ por $[x]$. Com estas notações podemos definir uma transformação $k$-linear

$$
\bar{\phi}: k[\Gamma, \mathcal{A}] \rightarrow k\left(\Gamma^{o p}, \mathcal{A}^{o p}\right)
$$

ao definir a imagem dos elementos da base natural de $k[\Gamma, \mathcal{A}]$, que é o conjunto dos $\mathcal{A}$-caminhos:

$$
\bar{\phi}\left(a_{0} \beta_{1} a_{1} \ldots a_{r-1} \beta_{r} a_{r}\right) \doteq\left[a_{r} \beta_{r} a_{r-1} \ldots a_{1} \beta_{1} a_{0}\right]
$$

para cada $\mathcal{A}$-caminho $a_{0} \beta_{1} a_{1} \ldots a_{r-1} \beta_{r} a_{r}$. Agora observamos que $\sim \subseteq \operatorname{ker} \bar{\phi}$. De fato:

$$
\begin{aligned}
& \bar{\phi}\left(a_{0} \beta_{1} a_{1} \ldots\left(a_{i}^{1}+\ldots+a_{i}^{s}\right) \ldots a_{r-1} \beta_{r} a_{r}-\sum_{j=1}^{s} a_{0} \beta_{1} a_{1} \ldots a_{i}^{j} \ldots a_{r-1} \beta_{r} a_{r}\right) \\
& =\bar{\phi}\left(a_{0} \beta_{1} a_{1} \ldots\left(a_{i}^{1}+\ldots+a_{i}^{s}\right) \ldots a_{r-1} \beta_{r} a_{r}\right)-\sum_{j=1}^{s} \bar{\phi}\left(a_{0} \beta_{1} a_{1} \ldots a_{i}^{j} \ldots a_{r-1} \beta_{r} a_{r}\right) \\
& \quad=\left[a_{r} \beta_{r} a_{r-1} \ldots\left(a_{i}^{1}+\ldots+a_{i}^{s}\right) \ldots a_{1} \beta_{1} a_{0}\right]-\sum_{j=1}^{s}\left[a_{r} \beta_{r} a_{r-1} \ldots a_{i}^{j} \ldots a_{1} \beta_{1} a_{0}\right]=0
\end{aligned}
$$

$\mathrm{e}$, para $\lambda \in k$,

$$
\begin{aligned}
\bar{\phi} & \left(a_{0} \beta_{1} a_{1} \ldots \lambda a_{i} \ldots a_{r-1} \beta_{r} a_{r}-\lambda\left(a_{0} \beta_{1} a_{1} \ldots a_{i} \ldots a_{r-1} \beta_{r} a_{r}\right)\right) \\
& =\bar{\phi}\left(a_{0} \beta_{1} a_{1} \ldots \lambda a_{i} \ldots a_{r-1} \beta_{r} a_{r}\right)-\lambda \bar{\phi}\left(a_{0} \beta_{1} a_{1} \ldots a_{i} \ldots a_{r-1} \beta_{r} a_{r}\right) \\
& =\left[a_{r} \beta_{r} a_{r-1} \ldots \lambda a_{i} \ldots a_{1} \beta_{1} a_{0}\right]-\lambda\left[a_{r} \beta_{r} a_{r-1} \ldots a_{i} \ldots a_{1} \beta_{1} a_{0}\right]=0
\end{aligned}
$$

Usando o Teorema do Homomorfismo, nós acabamos de provar que existe uma transformação $k$-linear

$$
\phi: k(\Gamma, \mathcal{A}) \rightarrow k\left(\Gamma^{o p}, \mathcal{A}^{o p}\right)
$$

que satisfaz

$$
\phi\left(\left[a_{0} \beta_{1} a_{1} \ldots a_{r-1} \beta_{r} a_{r}\right]\right)=\left[a_{r} \beta_{r} a_{r-1} \ldots a_{1} \beta_{1} a_{0}\right]
$$

É fácil se convencer de que $\phi$ é bijetora. Para concluir a demonstração da primeira parte do enunciado, resta mostrar que $\phi$ é um anti-homomorfismo de álgebras. É fácil ver que $\phi$ preserva o elemento identidade. Mostremos que ela antipreserva a multiplicação. Sejam $a=\left[a_{0} \beta_{1} a_{1} \ldots a_{r-1} \beta_{r} a_{r}\right]$ e $b=\left[b_{0} \gamma_{1} b_{1} \ldots b_{s-1} \gamma_{s} b_{s}\right]$ as classes de dois $\mathcal{A}$-caminhos. Se $e\left(\beta_{r}\right) \neq s\left(\gamma_{1}\right)$, é direto provar que $\phi(a b)=0=\phi(b) \phi(a)$. Então suponha que $e\left(\beta_{r}\right)=s\left(\gamma_{1}\right)$. Neste caso, 


$$
\begin{aligned}
\phi(a b) & =\phi\left(\left[a_{0} \beta_{1} a_{1} \ldots a_{r-1} \beta_{r} a_{r}\right]\left[b_{0} \gamma_{1} b_{1} \ldots b_{s-1} \gamma_{s} b_{s}\right]\right) \\
& =\phi\left(\left[a_{0} \beta_{1} a_{1} \ldots a_{r-1} \beta_{r}\left(a_{r} . b_{0}\right) \gamma_{1} b_{1} \ldots b_{s-1} \gamma_{s} b_{s}\right]\right) \\
& =\left[b_{s} \gamma_{s} b_{s-1} \ldots b_{1} \gamma_{1}\left(a_{r} . b_{0}\right) \beta_{r} a_{r-1} \ldots a_{1} \beta_{1} a_{0}\right] \\
& =\left[b_{s} \gamma_{s} b_{s-1} \ldots b_{1} \gamma_{1}\left(b_{0} \cdot{ }^{\prime} a_{r}\right) \beta_{r} a_{r-1} \ldots a_{1} \beta_{1} a_{0}\right] \\
& =\left[b_{s} \gamma_{s} b_{s-1} \ldots b_{1} \gamma_{1} b_{0}\right]\left[a_{r} \beta_{r} a_{r-1} \ldots a_{1} \beta_{1} a_{0}\right] \\
& =\phi\left(\left[b_{0} \gamma_{1} b_{1} \ldots b_{s-1} \gamma_{s} b_{s}\right]\right) \phi\left(\left[a_{0} \beta_{1} a_{1} \ldots a_{r-1} \beta_{r} a_{r}\right]\right)=\phi(b) \phi(a)
\end{aligned}
$$

Isso prova que $k(\Gamma, \mathcal{A})$ é anti-isomorfa a $k\left(\Gamma^{o p}, \mathcal{A}^{o p}\right)$ via $\phi$, o que equivale a dizer que $k(\Gamma, \mathcal{A})^{o p}$ é isomorfa a $k\left(\Gamma^{o p}, \mathcal{A}^{o p}\right)$, concluindo a primeira parte.

A segunda parte do enunciado segue diretamente da primeira, basta observar que a função $\phi$ definida acima satifaz $\phi(I)=I^{o p}$.

Proposição 5.5.2. Seja $\Lambda=k(\Gamma, \mathcal{A})$ como na Proposição 5.5.1. Denote por $D=\operatorname{Hom}_{k}(-, k)$ o funtor dualidade. Seja $M=\left(\left(M_{i}\right)_{i \in \Gamma_{0}},(\phi)_{\alpha \in \Gamma_{1}}\right)$ um $\Lambda$-módulo. Então $D M$ é um $\Lambda^{o p}$-módulo que corresponde a uma representação generalizada denotada por $D M=\left(\left((D M)_{i}\right)_{i \in \Gamma_{0}},\left(\psi_{\alpha}\right)_{\alpha \in \Gamma_{1}}\right)$. Daí existe, para cada $i \in \Gamma_{0}$, um isomorfismo de $A_{i}^{o p}$-módulos $f_{i}:(D M)_{i} \rightarrow D\left(M_{i}\right)$. Além disso, para cada flecha $\alpha: i \rightarrow j \in \Gamma_{1}$, o seguinte diagrama comuta:

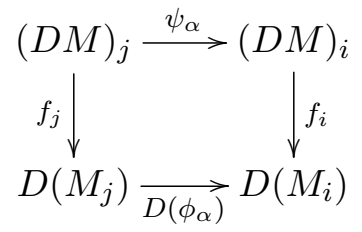

(isso significa que podemos fazer a identificação $D\left(\phi_{\alpha}\right)=\psi_{\alpha}$ ). Além disso, se $\Gamma$ tem um conjunto de relações $I$, então $D M$ satisfaz $I^{o p}$ se e somente se $M$ satisfaz $I$.

Demonstração. É útil lembrar como eram as equivalências quase inversas $F$ e $G$ discutidas na demonstração do Teorema 5.1.3.

Seja $i \in \Gamma_{0}$. Primeiro de tudo, note que

$$
\begin{gathered}
D M=\operatorname{hom}_{k}(M, k) \Rightarrow(D M)_{i}=1_{A_{i}}\left(\operatorname{hom}_{k}(M, k)\right) \\
D\left(M_{i}\right)=\operatorname{hom}_{k}\left(M_{i}, k\right)=\operatorname{hom}_{k}\left(M .1_{A_{i}}, k\right)
\end{gathered}
$$

Podemos definir

$$
\begin{gathered}
f_{i}: 1_{A_{i}} \operatorname{Hom}_{k}(M, k) \rightarrow \operatorname{Hom}_{k}\left(M .1_{A_{i}}, k\right) \\
1_{A_{i}} .\left.g \mapsto g\right|_{M .1_{A_{i}}}
\end{gathered}
$$

Agora verificamos que $f_{i}$ é um isomorfismo:

- É fácil ver que $f_{i}$ está bem definida e é $k$-linear. 
- $f_{i}$ é um morfismo de $A_{i}^{o p}$-módulos: sejam $g \in \operatorname{Hom}_{k}(M, k), a \in A_{i}^{o p}$ e $x \in M .1_{A_{i}}$. Temos que: $f_{i}\left(a .1_{A_{i}} g\right)(x)=\left.(a . g)\right|_{M .1_{A_{i}}}(x)=(a . g)(x)=g(x a)=g\left(x a 1_{A_{i}}\right)=\left.g\right|_{M .1_{A_{i}}}(x a)=$ $f_{i}\left(1_{A_{i}} g\right)(x a)=\left(a . f_{i}\left(1_{A_{i}} g\right)\right)(x) \Rightarrow f_{i}\left(a \cdot 1_{A_{i}} g\right)=a \cdot f_{i}\left(1_{A_{i}} g\right)$.

- $f_{i}$ é injetora: Suponha que $f_{i}\left(1_{A_{i}} g\right)=0$. Então $\left(1_{A_{i}} g\right)(x)=0$ para todo $x \in M .1_{A_{i}} \Rightarrow$ $\left(1_{A_{i}} \cdot g\right)\left(x \cdot 1_{A_{i}}\right)=0$ para todo $x \in M \Rightarrow g\left(x \cdot 1_{A_{i}}\right)=0$ para todo $x \in M \Rightarrow\left(1_{A_{i}} \cdot g\right)(x)=0$ para todo $x \in M \Rightarrow 1_{A_{i}} . g=0$.

- $f_{i}$ é sobrejetora: Seja $h \in \operatorname{Hom}_{k}\left(M .1_{A_{i}}, k\right)$. Sabemos que $M \cong \oplus_{j \in \Gamma_{0}} M .1_{A_{j}}$. Podemos assim definir uma transformação $k$-linear $g \in \operatorname{Hom}_{k}(M, k), g: \oplus_{j \in \Gamma_{0}} M .1_{A_{j}} \rightarrow k, g=\left(\delta_{j i} h\right)_{j \in \Gamma_{0}}$, onde $\delta_{j i}$ é um delta de Kronecker. Então, se $x \in M .1_{A_{i}}, f_{i}\left(1_{A_{i}} . g\right)(x)=\left.g\right|_{M .1_{A_{i}}}(x)=h(x)$. Assim $f_{i}\left(1_{A_{i}} \cdot g\right)=h$.

Provamos portanto que $f_{i}$ é um isomorfismo de $A_{i}$-módulos. O próximo passo é mostrar a comutatividade do diagrama do enunciado. Sejam $g \in \operatorname{Hom}_{k}(M, k)$ e $x \in M$. Então:

$$
\begin{aligned}
\left(f_{i} \circ \psi_{\alpha}\right)\left(1_{A_{j}} . g\right) & \left(x .1_{A_{i}}\right)=f_{i}\left(\psi_{\alpha}\left(1_{A_{j}} . g\right)\right)\left(x .1_{A_{i}}\right)=f_{i}\left(1_{A_{i}} \alpha g\right)\left(x .1_{A_{i}}\right)=\left.(\alpha g)\right|_{M .1_{A_{i}}}\left(x .1_{A_{i}}\right) \\
& =(\alpha g)\left(x .1_{A_{i}}\right)=g(x \alpha)=\left.g\right|_{M .1_{A_{j}}}\left(x \alpha 1_{A_{j}}\right)=\left.g\right|_{M .1_{A_{j}}}(x \alpha)=\left.g\right|_{M .1_{A_{j}}}\left(\phi_{\alpha}\left(x .1_{A_{i}}\right)\right) \\
& \left.=D\left(\phi_{\alpha}\right)\left(\left.g\right|_{M .1_{A_{j}}}\right)\left(x .1_{A_{i}}\right)=D\left(\phi_{\alpha}\right)\left(f_{j}\left(1_{A_{i}} \cdot g\right)\right)\left(x .1_{A_{i}}\right)\right) \\
& =\left(D\left(\phi_{\alpha}\right) \circ f_{j}\right)\left(1_{A_{i}} \cdot g\right)\left(x .1_{A_{i}}\right)
\end{aligned}
$$

Logo $\left(f_{i} \circ \psi_{\alpha}\right)=\left(D\left(\phi_{\alpha}\right) \circ f_{j}\right)$, como queríamos.

A última afirmação do enunciado é verificada diretamente, lembrando que $D$ é um funtor $k$-linear fiel e pleno.

Voltemos a denotar por $\Lambda$ uma ACGR $k(\Gamma, \mathcal{A}, I)$. Os resultados desta subseção nos permitem obter uma terceira forma de realizar um $A_{i}$-módulo como um $\Lambda$-módulo, onde $i \in \Gamma_{0}$. (As outras duas foram discutidas na Seção 5.3).

Definição 5.5.3. Seja $i \in \Gamma_{0}$, e seja $M$ um $A_{i}$-módulo. Então $D(M)$ é um $A_{i}^{o p}$-módulo, e portanto o cone $\mathscr{C}_{i}(D M)$ é um $\Lambda^{o p}$-módulo. Finalmente, $D\left(\mathscr{C}_{i}(D M)\right)$ é um $\Lambda$-módulo, que chamamos de cone dual de $M$. Vamos usar a notação $\mathscr{C}^{*}{ }_{i}(M) \doteq D\left(\mathscr{C}_{i}(D M)\right)$.

Proposição 5.5.4. Dados dois $A_{i}$-módulos $M$ e $N, \mathscr{C}^{*}{ }_{i}(M \oplus N) \cong \mathscr{C}^{*}{ }_{i}(M) \oplus \mathscr{C}^{*}{ }_{i}(N)$.

Demonstração. Isso segue porque o funtor dualidade preserva somas diretas e porque $\mathscr{C}_{i}$ também preserva somas diretas por causa da Proposição 5.3.2.

Proposição 5.5.5. Se $I$ é um $A_{i}$-módulo injetivo, então $\mathscr{C}^{*}{ }_{i}(I)$ é um $\Lambda$-módulo injetivo.

Demonstração. Como $I$ é um $A_{i}$-módulo injetivo e $D$ é uma dualidade, $D I$ é um $A_{i}^{o p}$-módulo projetivo. Por causa da Proposição 5.3.3, $\mathscr{C}_{i}(D I)$ é um $\Lambda^{o p}$-módulo projetivo, e de novo porque $D$ é uma dualidade, $\mathscr{C}^{*}{ }_{i}(I)=D\left(\mathscr{C}_{i}(D I)\right)$ é um $\Lambda$-módulo injetivo.

Observação 5.5.6. Na Seção 5.3, demos uma descrição das representações associadas a cones. Ou seja, já sabemos como calcular cones. Graças à Proposição 5.5.2, calcular cones duais não trará uma dificuldade maior que a de calcular cones: dado $M$ um $A_{i}$-módulo, nós calculamos o cone de $D M$ 
sobre $\left(\Gamma^{o p}, \mathcal{A}^{o p}, I^{o p}\right)$ e daí obtemos o cone dual de $M$ sobre $(\Gamma, \mathcal{A}, I)$ usando a Proposição 5.5.2. Esta proposição nos diz que o que precisamos fazer é tomar os duais dos módulos em cada vértice e tomar a transformação linear transposta em cada flecha, o que na prática é feito transpondo matrizes. Exemplos práticos disso vão surgir na Seção 5.6.

\subsection{Módulos injetivos}

Nesta seção vamos dar uma descrição das representações associadas a módulos injetivos. Como vamos ver, os módulos injetivos serão casos particulares de cones duais, em analogia com os projetivos, que eram casos particulares de cones, conforme vimos na Seção 5.4.

Para cada $i \in \Gamma_{0}$, seja $E_{i}=\left\{e_{i 1}, \ldots, e_{i s_{i}}\right\}$ um conjunto completo de idempotentes primitivos dois a dois ortogonais em $A_{i}$. Se $D: \bmod A_{i}^{o p} \rightarrow \bmod A_{i}$ é o funtor dualidade, então conforme o Teorema 2.2.12, um conjunto completo de classes de isomorfismo distintas de $A_{i}$-módulos injetivos indecomponíveis é dado por $I_{i}^{1}=D\left(A_{i} e_{i 1}\right), \ldots, I_{i}^{s_{i}}=D\left(A_{i} e_{i s_{i}}\right)$.

Por outro lado, se $E=\left\{\overline{e_{i j}}: i \in \Gamma_{0}, 1 \leq j \leq s_{i}\right\}$, então $E$ é um conjunto completo de idempotentes primitivos dois a dois ortogonais em $\Lambda$. Isso significa que um conjunto completo de classes de isomorfismo distintas de $\Lambda$-módulos injetivos indecomponíveis é dado por $\{I(i, j): i \in$ $\left.\Gamma_{0}, 1 \leq j \leq s_{i}\right\}$, onde $I(i, j)=D\left(\Lambda \overline{e_{i j}}\right)$.

Proposição 5.6.1. Com as notações acima, $\mathscr{C}^{*}{ }_{i}\left(I_{i}^{j}\right) \cong I(i, j)$.

Demonstração.

$$
\mathscr{C}^{*}{ }_{i}\left(I_{i}^{j}\right)=D\left(\mathscr{C}_{i}\left(D\left(I_{i}^{j}\right)\right)\right)=D\left(\mathscr{C}_{i}\left(D\left(D\left(A_{i} e_{i j}\right)\right)\right)\right) \cong D\left(\mathscr{C}_{i}\left(A_{i} e_{i j}\right)\right)=D\left(\Lambda \overline{e_{i j}}\right)=I(i, j)
$$

onde a penúltima igualdade segue da Proposição 5.4.1.

A Proposição 5.6.1 dá uma descrição completa $\Lambda$-módulos injetivos indecomponíveis. Para efetuar os cálculos desses módulos em exemplos práticos, precisamos combinar essa descrição com a Observação 5.5.6 acima.

Com isso é direto verificar a próxima proposição, que reflete o caso particular desta construção quando não há relações:

Proposição 5.6.2. Suponha que $I=0$. Seja $I(i, j)=\left(\left(M_{i}\right)_{i \in \Gamma_{0}},\left(\phi_{\alpha}\right)_{\alpha \in \Gamma_{0}}\right)$ a representação associada a $I(i, j)$. Então, para $l \in \Gamma_{0}$,

Se $l=i$, então $M_{l}=M_{i}=I_{i}^{j}$.

Se $l \neq i$, denote

$$
n_{l}=\sum_{\gamma: l=i_{0} \rightarrow i_{1} \rightarrow \ldots \rightarrow i_{r}=i}\left(\operatorname{dim}_{\mathrm{k}} A_{i_{1}}\right) \ldots\left(\operatorname{dim}_{\mathrm{k}} A_{i_{r-1}}\right)\left(\operatorname{dim}_{\mathrm{k}} I_{i}^{j}\right) .
$$

onde $\gamma$ percorre todos os possíveis caminhos $l \rightsquigarrow i$. Então $M_{l} \cong\left(A_{l}^{*}\right)^{n_{l}}$ como $A_{l}$-módulos, onde denotamos $A_{l}^{*}=D\left(A_{l}\right)$ por brevidade. Em particular, se não há caminhos $l \rightsquigarrow i$, então $M_{l}=0$.

Exemplo 5.6.3. Seja $A$ a álgebra de caminhos dada pela aljava

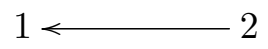


Então há dois $A$-módulos injetivos indecomponíveis, a saber,

$$
I_{1}: k \longleftarrow i d=k
$$

Agora seja $\Lambda$ a ACG dada por

$$
A \longleftarrow A
$$

Queremos calcular os $\Lambda$-módulos injetivos indecomponíveis. De acordo com as discussões feitas acima, primeiro calculamos os projetivos indecomponíveis sobre a ACG dada por

$$
A^{o p} \longrightarrow A^{o p}
$$

e notamos que $A^{o p}$ é a álgebra de caminhos sobre a aljava

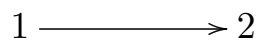

Neste caso, o cálculo já foi feito no Exemplo 5.4.3. Portanto só resta aplicar a Proposição 5.5.2. Portanto os $\Lambda$-módulos injetivos indecomponíveis são:

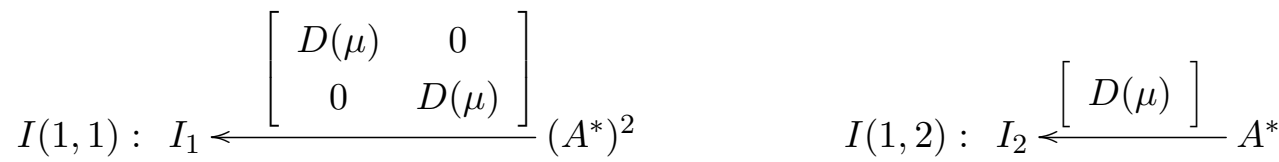

$$
\begin{aligned}
& I(2,1): 0 \longleftarrow I_{1} \quad I(2,2): 0 \longleftarrow I_{2}
\end{aligned}
$$




\section{Apêndice A}

\section{Conceitos equivalentes aos de ACG}

Dedicamos esta parte para mencionar alguns conceitos similares a, equivalentes a ou generalizações de álgebras de caminhos generalizadas que foram encontrados na literatura.

\section{A.1 Pró-espécies}

As pró-espécies foram introduzidas por Julian Külshammer em [9]. Este conceito é uma generalização de outro mais conhecido, que é o de espécies, introduzidas por P. Gabriel em [8].

As pró-espécies têm com as espécies uma relação análoga à das álgebras de caminhos generalizadas com as álgebras de caminhos ordinárias: os anéis de divisão das espécies são trocados por álgebras em geral, e os bimódulos entre os anéis de divisão são trocados por bimódulos projetivos em ambos os lados, conforme enunciaremos abaixo.

Outro comentário antes de colocar a definição é que no artigo original [9], o autor recorre à linguagem das bicategorias. Preferimos abordar o conceito com uma linguagem mais simples para tornar mais próximo com as notações aqui empregadas.

Definição A.1.1. Dada uma aljava $\Gamma$, uma pró-espécie de álgebras $\Lambda$ sobre $\Gamma$ consiste nas seguintes informações:

- Para cada vértice $i \in \Gamma_{0}$ associamos uma álgebra $\Lambda(i)$.

- Para cada flecha $\alpha: i \rightarrow j \in \Gamma_{1}$, associamos um $\Lambda(i)-\Lambda(j)$-bimódulo que é projetivo e finitamente gerado em ambos os lados, denotado por $\Lambda(\alpha)$.

Além disso, se todos os $\Lambda(\alpha)$ forem livres de posto finito em ambos os lados, $\Lambda$ é chamada de espécie de álgebras.

Definição A.1.2. Seja $\Gamma$ uma aljava e seja $\Lambda$ uma pró-espécie sobre $\Gamma$. Então uma representação $M$ de $\Lambda$ consiste nas seguintes informações:

- Para cada vértice $i \in \Gamma_{0}$ associamos um $\Lambda(i)$-módulo, denotado por $M(i)$.

- Para cada flecha $\alpha: i \rightarrow j \in \Gamma_{1}$ associamos um morfismo de $\Lambda(j)$-módulos denotado por $M(\alpha): M(i) \otimes_{\Lambda(i)} \Lambda(\alpha) \rightarrow M(j)$. 
Além disso, se $M$ e $N$ são representações de $\Lambda$, um morfismo de representações consiste em uma tupla $f=(f(i))_{i \in \Gamma_{0}}$, onde cada $f(i): M(i) \rightarrow N(i)$ é um morfismo de $\Lambda(i)$-módulos, tal que para toda flecha $\alpha: i \rightarrow j \in \Gamma_{1}$ o seguinte diagrama é comutativo:

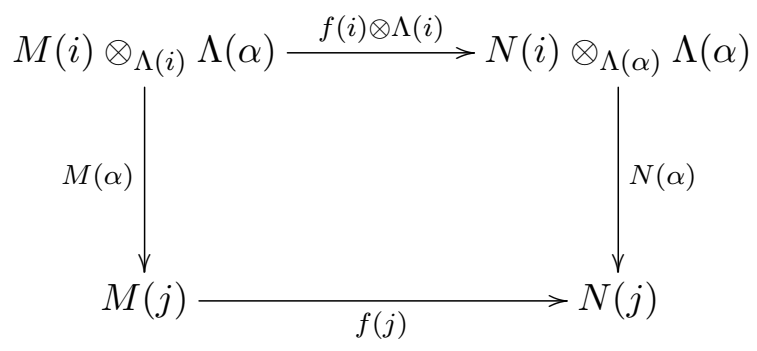

Definição A.1.3. Seja $\Lambda$ uma pró-espécie sobre $\Gamma$. Então, dado um caminho $\gamma=\alpha_{1} \ldots \alpha_{t}$ de comprimento não nulo sobre $\Gamma$, denotamos $\Lambda(\gamma)=\Lambda\left(\alpha_{1}\right) \otimes_{s\left(\alpha_{2}\right)} \Lambda\left(\alpha_{2}\right) \ldots \otimes_{s\left(\alpha_{t}\right)} \Lambda\left(\alpha_{t}\right)$. Daí definimos a álgebra tensorial de $\Lambda$ por

$$
T(\Lambda)=\left(\prod_{i \in \Gamma_{0}} \Lambda(i)\right) \oplus\left(\bigoplus_{\gamma \text { caminho sobre } \Gamma} \Lambda(\gamma)\right)
$$

A multiplicação de $T(\Lambda)$ fica determinada pelas seguintes informações:

- A multiplicação dentro de $\prod_{i \in \Gamma_{0}} \Lambda(i)$ é a usual.

- A multiplicação de um elemento de $\Lambda(i)$ por um de $\Lambda(\gamma)$ é a induzida da estrutura de módulo se $s(\gamma)=i$, e é zero caso contrário. Simétrico para o outro lado.

- Se $x \in \Lambda(\gamma)$ e $y \in \Lambda(\eta)$ então $x y=x \otimes y$ se $e(\gamma)=s(\eta)$ e $x y=0$ caso contrário.

Observação A.1.4. Note que, se $\Lambda$ é uma pró-espécie sobre $Q$ tal que $\Lambda(\alpha)$ é um bimódulo livre de posto 1 para cada flecha $\alpha$, então $T(\Lambda)$ é uma ACG. De fato, $T(\Lambda) \cong k\left(\Gamma,\{\Lambda(i)\}_{i}\right)$.

O próximo teorema é um equivalente do Teorema 5.1.3 para pro-espécies:

Teorema A.1.5. Seja $\Lambda$ uma pró-espécie sobre $\Gamma$. Então as categorias de representações sobre $\Lambda$ e de módulos sobre $T(\Lambda)$ são equivalentes.

Para terminar esta seção, mencionamos que o artigo [9] também discute um equivalente do Teorema 4.2.3 para pró-espécies.

\section{A.2 Álgebras de matrizes triangulares}

A conexão entre as álgebras de caminhos generalizadas e as álgebras de matrizes triangulares é discutida, por exemplo, em [12] e [13]. Vamos definir aqui o que se entende por álgebras de matrizes triangulares e indicar qual é a relação desse conceito com o de ACG's.

Definição A.2.1. Seja $n$ um número natural. Para cada $i$ entre 1 e $n$, seja $A_{i}$ uma álgebra. Para cada par de índices $1 \leq i<j \leq n$, seja $B_{i j}$ um $\left(A_{i}-A_{j}\right)$-bimódulo. Considere, para $1 \leq i<j \leq n$, o seguinte bimódulo: 


$$
A_{i j}=B_{i j} \oplus\left(\oplus_{l=1}^{j-i-1} \oplus_{i<k_{1}<k_{2}<\ldots<k_{l}<j} B_{i k_{1}} \otimes_{A_{k_{1}}} B_{k_{1} k_{2}} \otimes_{A_{k_{2}}} \ldots \otimes_{A_{k_{l}}} B_{k_{l} j}\right)
$$

Para cada tripla de índices $1 \leq i<j<q \leq n$, temos uma inclusão natural $\mu_{i j q}: A_{i j} \otimes A_{j q} \rightarrow A_{i q}$, portanto fica definida, de modo evidente, a seguinte álgebra:

$$
\Lambda=\left(\begin{array}{cccc}
A_{1} & A_{12} & \cdots & A_{1 n} \\
0 & A_{2} & \cdots & A_{2 n} \\
\vdots & \vdots & \ddots & \vdots \\
0 & 0 & \cdots & A_{n}
\end{array}\right)
$$

Considere ainda as seguintes propriedades:

1. Para cada par de índices $1 \leq i<j \leq n, B_{i j}$ é livre como $A_{i}$-módulo à esquerda e livre como $A_{j}$-módulo à direita.

2. Para cada $i<j, \operatorname{Hom}_{A_{i}}\left(B_{i j}, A_{i}\right)$ é projetivo ou injetivo como $A_{j}$-módulo.

3. Para cada $i<j$, temos um isomorfismo de $\left(A_{j}-A_{i}\right)$-bimódulos $\operatorname{Hom}_{A_{i}}\left(B_{i j}, A_{i}\right) \cong \operatorname{Hom}_{A_{j}}\left(B_{i j}, A_{j}\right)$.

4. Para cada $i$ entre 1 e $n$, a álgebra $A_{i}$ é de Frobenius. (Isso significa que $A$ e $D A$ são isomorfos como $A$-módulos à esquerda, onde $D$ denota o funtor dualidade).

Então, se $\Lambda$ satisfaz (1) e (2), seguindo [12], dizemos que $\Lambda$ é uma álgebra de matrizes triangulares superiores normalmente generalizada. Se $\Lambda$ satisfaz (1),(3) e (4), seguindo [13], dizemos que $\Lambda$ é uma álgebra de matrizes triangulares de tipo Frobenius.

Exemplo A.2.2. Seja $\Gamma$ uma aljava acíclica. Podemos então supor, sem perda de generalidade, que $\Gamma_{0}=\{1, \ldots, n\}$ e que $i<j$ toda vez que existir uma flecha da forma $i \rightarrow j$. Seja $\mathcal{A}=\left\{A_{1}, \ldots, A_{n}\right\}$ uma família de álgebras. Seja, para cada par de vértices $1 \leq i<j \leq n, B_{i j}$ o $k$-espaço vetorial gerado pelo seguinte conjunto:

$$
\left\{a \alpha b \in k(\Gamma, \mathcal{A}): a \in A_{i}, b \in A_{j} \text { e } \alpha: i \rightarrow j \text { é uma flecha em } \Gamma_{1}\right\}
$$

Então a álgebra de matrizes triangulares obtida a partir dos $A_{i}$ 's e dos $B_{i j}$ 's tal como na Definição A.2.1 acima é isomorfa a $k(\Gamma, \mathcal{A})$. Além disso, $k(\Gamma, \mathcal{A})$ é normalmente generalizada. Se, ainda, cada $A_{i}$ é uma álgebra de Frobenius, então $k(\Gamma, \mathcal{A})$ é de tipo Frobenius.

Observação A.2.3. Segundo os autores de [12], a principal motivação para introduzir as álgebras de matrizes triangulares superiores normalmente generalizadas é poder dar um tratamento unificado entre as álgebras de caminhos generalizadas e as álgebras de aljavas sobre uma álgebra, um conceito não incluído aqui mas que é relembrado no referido artigo.

Para encerrar esta seção, vamos dizer como podemos definir as representações neste contexto de álgebras de matrizes triangulares.

Definição A.2.4. Sejam $A_{i}, B_{i j}, A_{i j}, \mu_{i j q}, \Lambda$ como na Definição A.2.1. (Para simplificar a notação a seguir, defina $\left.A_{i i}=A_{i}\right)$. Uma representação sobre $\Lambda$ é uma $n$-upla 


$$
X=\left(\begin{array}{c}
X_{1} \\
X_{2} \\
\vdots \\
X_{n}
\end{array}\right)
$$

onde cada $X_{i}$ é um $A_{i}$-módulo à esquerda, junto com uma família de morfismos de $A_{i}$-módulos $\phi_{i j}: A_{i j} \otimes_{A_{j}} X_{j} \rightarrow X_{i}$ para cada par de índices $1 \leq i<j \leq n$, tal que cada diagrama como abaixo é comutativo:

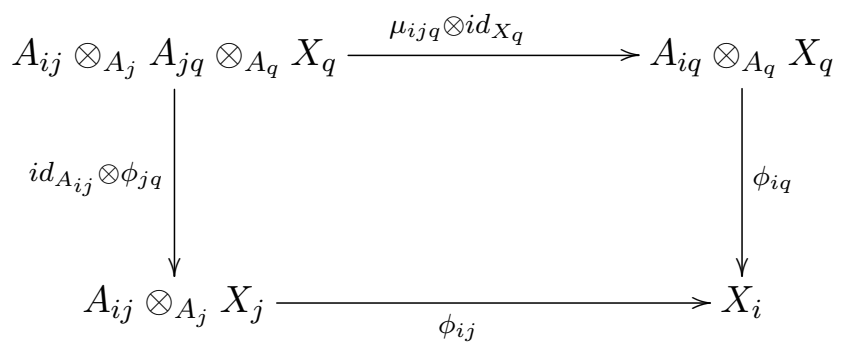

Além disso, um morfismo entre duas representações $X$ e $Y$ é uma $n$-upla $f=\left(f_{i}\right)_{1 \leq i \leq n}$ : $X \rightarrow Y$, onde cada $f_{i}: X_{i} \rightarrow Y_{i}$ é um morfismo de $A_{i}$-módulos, e tal que para cada par $i<j$ vale a relação $f_{i} \phi_{i j}^{X}=\phi_{i j}^{Y}\left(i d_{A_{i j}} \otimes f_{j}\right)$.

\section{A.3 Álgebras de pseudocaminhos (ou pseudotensoriais)}

As álgebras de pseudocaminhos foram introduzidas por Fang Li em [10]. O que motivou a introdução desse conceito foi a tentativa de estabelecer um teorema análogo ao de Gabriel (Teorema 2.6.1) para álgebras sobre corpos não necessariamente algebricamente fechados. Como mostra Fang Li no artigo citado acima, tal resultado análogo não existe em geral para álgebras de caminhos generalizadas, mas existe para álgebras de pseudocaminhos.

Assim como as álgebras de caminhos generalizadas podem ser vistas como álgebras tensoriais, também as álgebras de pseudocaminhos, que generalizam as ACG's, podem ser vistas como álgebras pseudotensoriais, no sentido definido por F. Li.

Definição A.3.1. Sejam $\Gamma$ uma aljava e $\mathcal{A}=\left\{A_{i}: i \in \Gamma_{0}\right\}$ uma família de álgebras. Então um $\mathcal{A}$-pseudocaminho de comprimento 0 é um elemento do conjunto $\cup_{i \in \Gamma_{0}} A_{i}$.

Um $\mathcal{A}$-pseudocaminho puro de comprimento de $n$ sobre $\Gamma$ é uma sequência formal da forma

$$
\left(a_{1} \beta_{1} b_{1}\right) \cdot\left(a_{2} \beta_{2} b_{2}\right) \ldots\left(a_{n} \beta b_{n}\right)
$$

onde $\beta_{1} \beta_{2} \ldots \beta_{n}$ é um caminho ordinário sobre $\Gamma$, e para todo $1 \leq j \leq n, a_{j} \in A_{s\left(\beta_{j}\right)}$ e $b_{j} \in A_{e\left(\beta_{j}\right)}$. Um $\mathcal{A}$-pseudocaminho geral de comprimento $n$ sobre $\Gamma$ é uma sequência formal que tem uma das seguintes formas: 


$$
\begin{aligned}
& \alpha_{1} \cdot c_{1} \cdot \alpha_{2} \cdot c_{2} \ldots \ldots c_{k} \cdot \alpha_{k} \\
& c_{0} \cdot \alpha_{1} \cdot c_{1} \cdot \alpha_{2} \cdot c_{2} \ldots \ldots c_{k} \cdot \alpha_{k} \\
& \alpha_{1} \cdot c_{1} \cdot \alpha_{2} \cdot c_{2} \ldots \ldots c_{k} \cdot \alpha_{k} \cdot c_{k+1} \\
& c_{0} \cdot \alpha_{1} \cdot c_{1} \cdot \alpha_{2} \cdot c_{2} \ldots . c_{k} \cdot \alpha_{k} \cdot c_{k+1}
\end{aligned}
$$

onde, para cada $j, \alpha_{j}$ é um $\mathcal{A}$-pseudocaminho puro, $c_{j} \in A_{e\left(\alpha_{j}\right)}, c_{0} \in A_{s\left(\alpha_{j}\right)}$ e $\alpha_{j}$ termina onde $\alpha_{j+1}$ começa. Além disso, $n=\sum_{j} l\left(\alpha_{j}\right)$.

O $k$-espaço vetorial tendo como base todos os $\mathcal{A}$-pseudocaminhos gerais sobre $\Gamma$ é denotado por $\operatorname{PSE}_{k}[\Gamma, \mathcal{A}]$. A álgebra de pseudocaminhos sobre o par $(\Gamma, \mathcal{A})$, denotada por $\operatorname{PSE}_{k}(\Gamma, \mathcal{A})$, é o espaço vetorial quociente de $\operatorname{PSE}_{k}[\Gamma, \mathcal{A}]$ por relações de linearidade e homogeneidade análogas às da definição de ACG. Também como as $A C G$ 's, a multiplicação de $\operatorname{PSE}_{k}(\Gamma, \mathcal{A})$ é definida por concatenação de pseudocaminhos.

Definição A.3.2. Seja $A$ uma álgebra (não necessariamente de dimensão finita sobre um corpo algebricamente fechado). Denote por $\pi: A \rightarrow A / \operatorname{rad} A$ a projeção canônica. Dizemos que o quociente $A / \operatorname{rad} A$ tem um levantamento se existe um homomorfismo $\epsilon: A / \operatorname{rad} A \rightarrow A$ tal que $\pi \epsilon=i d$. (Intuitivamente, é como se o Teorema de Wedderburn-Malcev fosse válido para $A$ ).

Como já mencionado, no artigo [10], o autor traz um exemplo, devido a W. Crawley-Boevey, de uma álgebra $A$ de dimensão finita sobre um corpo $k$ tal que $A / \operatorname{rad} A$ tem um levantamento mas tal que não existe um análogo do Teorema de Gabriel para ACG's que valha para $A$, ou seja, tal que $A$ não é isomorfa a um quociente da álgebra tensorial $T\left(A / \mathrm{rad} A, \operatorname{rad} A / \operatorname{rad}^{2} A\right)$. É relevante mencionar que o corpo $k$ considerado neste exemplo é o corpo $\mathbb{Z}_{2}(t)$ das funções racionais com coeficientes sobre o corpo de dois elementos $\mathbb{Z}_{2}$.

No sentido de generalizar o Teorema de Gabriel para corpos que não sejam algebricamente fechados, as álgebras de pseudocaminhos são um pouco mais eficazes do que as de caminhos generalizadas, conforme mostra o Teorema abaixo, que é o resultado principal de [10]. (É importante mencionar que o conceito de relações no teorema é um pouco diferente do conceito tratado aqui).

Teorema A.3.3. Seja $A$ uma álgebra artiniana sobre um corpo $k$ tal que o quociente $A / \operatorname{rad} A$ tem um levantamento.

- Existem uma aljava $\Gamma$, uma família de álgebras $\mathcal{A}=\left\{A_{i}: i \in \Gamma_{0}\right\}$ e um conjunto de relações $\rho$ tais que $A \cong \operatorname{PSE}_{k}(\Gamma, \mathcal{A}, \rho), \operatorname{com} \operatorname{rad}^{s} A \subseteq(\rho) \subseteq \operatorname{rad} A$ para algum $s \geq 1$.

- Se além disso $A$ é de dimensão finita e $\operatorname{rad}^{2} A=0$, então existem uma aljava $\Gamma$, uma família de álgebras $\mathcal{A}=\left\{A_{i}: i \in \Gamma_{0}\right\}$ e um conjunto de relações $\rho$ tais que $A \cong k(\Gamma, \mathcal{A}, \rho)$. (E vale também uma condição análoga a $(\rho)$ ser um ideal admissível). 


\section{Índice}

$\mathcal{A}$-caminho, 23

oposta, 13

álgebra, 3

básica, 5,12

ordinária, 18

quociente, 15

de Kronecker, 5

reduzida, 40

de matrizes, 5

de matrizes triangulares

de tipo Frobenius, 67

normalmente generalizada, 67

de polinômios, 5

de pseudocaminhos, 69

dimensão finita, 4

epimorfismo, 5

homomorfismo, 5

isomorfismo, 5

monomorfismo, 5

oposta, 7

tensorial, 66

álgebra de caminhos, 16, 25

com relações, 18

ordinária, 16

álgebra de caminhos com relações, 39

álgebra de caminhos generalizada, 23

com relações, 31

bimódulo, 8

blowing-up, 47

caminho, 13

induzido no quociente, 40

começo, 13

composição, 16

ordinário, 13

subjacente, 25

término, 13

ciclo orientado, 13

coerente com flechas, 40

compatível, 41

completo, 11

cone, 55

dual, 62

dimensão

finita, 4

dualidade, 8

$\mathrm{AC}$, ver álgebra de caminhos

ACG, ver álgebra de caminhos generalizada

espécie, 65

ACGR, ver álgebra de caminhos generalizada com relações

ACR, ver álgebra de caminhos com relações

algebricamente fechado, 4

aljava, 13

de Gabriel, 18

acíclica, 13

conexa, 14

finita, 13

morfismos, 15

flecha, 13

começo, 13

múltipla, 13

término, 13

fonte, 13

funtor

dualidade, 8

inclusão, 55

grupo de Grothendieck, 12 
ideal

admissível, 17

idempotente, 11

inclusão, 55

injetivo, 9

levantamento, 69

loop, 13

módulo, 5

epimorfismo, 7

indecomponível, 11

isomorfismo, 7

monomorfismo, 7

morfismo, 6

quociente, 7

simples, 11

submódulo, 7

multiplicação, 3,5

ortogonal, 11

passeio, 14

poço, 13

pró-espécie, 65

primitivo, 11

projetivo, 9

pseudocaminho, 68

quasirregular, 11

quiver, 13

radical, 10

relação, 17, 31

compatível, 41

externa, 41

interna, 41

representação(ões), 20, 50

de álgebras de matrizes triangulares, 67

de pró-espécie, 65

finitamente gerada, 20, 50

generalizada, 50

morfismos entre, 20, 50, 66

satisfazendo relações, 20,50

rotulagem, 40

simplificável, 40 sem relações, 40

simplificação, 39

equivalente, 39

sem ciclos, 39

sem relações, 39

trivial, 39

soma, 3,5

subaljava, 14

plena, 14

submódulo, 7

vértice, 13

fonte, 13

poço, 13 


\section{Bibliografia}

[1] Frank W Anderson and Kent R Fuller. Rings and Categories of Modules, volume 13 of Graduate Texts in Mathematics. Springer, 1992.

[2] Ibrahim Assem. Algèbres et modules. Les Presses de l'Université d'Ottawa, Masson, 1997.

[3] Ibrahim Assem and Flávio U Coelho. Basic Representation Theory of Algebras, volume 283 of Graduate Texts in Mathematics. Springer, 2020.

[4] Maurice Auslander, Idun Reiten, and Sverre Smalø. Representation Theory of Artin Algebras, volume 36 of Cambridge Studies in Advanced Mathematics. Cambridge University Press, jan 1995.

[5] Thomas Brüstle. Derived-tame Tree Algebras. Compositio Mathematica, 129(3):301-323, 2001.

[6] Rosa M Ibáñez Cobos, Gabriel Navarro, and Javier López Peña. A note on generalized path algebras. Rev. Roumaine Math. Pures Appl., 53(1):25-36, 2008.

[7] Flávio U Coelho and Shao-Xue Liu. Generalized path algebras. In Interaction between ring theory and representations of algebras. Marcel Dekker, 2000.

[8] Peter Gabriel. Indecomposable representations II. In Symposia Mathematica, Vol XI. Convegno di Algebra Comutativa, INDAM, Rome, 1971, pages 81-104. Academic Press, London, 1973.

[9] Julian Külshammer. Pro-Species of Algebras I: Basic Properties. Algebr. Represent. Theor., $20: 1215-1238,2017$.

[10] Fang Li. Characterization of left artinian algebras through pseudo path algebras. J. Aust. Math. Soc., 83(3):385-416, 2007.

[11] Fang Li. Modulation and natural valued quiver of an algebra. Pacific. J. Math., 256(1):105-128, 2012.

[12] Fang Li and Chang Ye. Gorenstein projective modules over a class of generalized matrix algebras and their applications. Algebra and Representation Theory, 18:693-710, 2015.

[13] Fang Li and Chang Ye. Representations of Frobenius-type Triangular Matrix Algebras. Acta Math. Sinica (English Series), 33(3):341-361, 2017.

[14] Joseph J Rotman. Advanced Modern Algebra (3rd edition), volume 180 of Graduate Studies in Mathematics. American Mathematical Society, 2017. 
[15] Wang Shugui. The generalized path algebras over standardly stratified algebras. Algebra Discrete Math., 3:119-126, 2006. 